Noncoding RNA mediated regulation of gene expression in disease and development

\author{
Adam Christopher Mueller
}

BS Biology, University of Maryland Baltimore County 2002

MS Applied Molecular Biology, University of Maryland Baltimore County 2003

A dissertation presented to the Graduate Faculty of the University of Virginia in Candidacy for the degree of Doctor of Philosophy

Department of Biochemistry and Molecular Genetics

University of Virginia

June, 2013 


\section{Table of Contents}

I. Abstract

II. Acknowledgements vii

1. Introduction 1-1

a. Dissertation Overview $1-2$

b. microRNA mediated regulation of the 1-3

DNA damage response and the implications

of such regulation for cancer.

i. Mechanisms of double strand 1-3

break repair

ii. MicroRNA biosynthesis and 1-5

regulatory action

iii. Regulation of microRNA expression $\quad 1-5$

during the DNA damage response

iv. MicroRNAs upregulated following 1-6

DNA damage

v. MicroRNAs downregulated following 1-6

DNA damage

vi. MicroRNAs with variable expression $\quad$ 1-7

following DNA damage

vii. MicroRNAs targeting DNA damage sensing 1-8

and repair proteins

viii. microRNAs targeting checkpoint activation $\quad 1-9$

proteins

ix. microRNAs on the frontier of cancer $1-10$

diagnosis and treatment

c. The role of Long Non-coding RNAs in regulating 1-11

gene expression

i. Xist mediated silencing of the 1-11

inactive $\mathrm{X}$-chromosome

ii. Pro-metastatic lncRNA HOTAIR and 1-11

PRC recruitment

iii. lncRNAs with cis enhancer-like functions $1-12$

iv. Trans-acting enhancer lncRNAs 1-15

v. MALAT1 regulation of gene splicing 1-16

vi. lncRNA mediated suppression of 1-17

microRNA mediated mRNA decay

vii. Challenges of future study of lncRNAs 1-18

in higher eukaryotes

d. Regulation of skeletal muscle development 1-19

i. The genetics of skeletal myogenesis 1-20

ii. MicroRNA regulation of skeletal 1-23

muscle development

iii. The role of long noncoding RNAs 1-23

in skeletal muscle development

e. Goals of Current Research 
i. The role of microRNAs in the DNA damage response

ii. The role of long noncoding RNAs in $\quad 1-26$

f. References skeletal myogenesis

2. The miR-99 family regulates the DNA damage 2-1 response through its target SNF2H.

a. Abstract 2-1

b. Body 2-2

c. References 2-19

d. Figures 2-22

3. MUNC: A lncRNA that facilitates the function of 3-1 MyoD in skeletal myogenesis

a. Abstract 3-1

b. Body 3-3

c. References 3-13

d. Figures 3-15

4. Summary and Future Directions 4-1

a. microRNA mediated regulation of the DNA 4-1

damage response and the implications of such regulation for cancer.

i. microRNA expression can mediate radiation sensitivity in cancer and microRNA expression changes that occur following exposure to radiation can affect cells' ability to repair DNA damage.

ii. MicroRNAs whose expression correlates with cells' DNA damage sensitivity and are upregulated following irradiation

iii. Regulation of miR-99 family in response to radiation and hormonal stimulus

iv. microRNAs that target chromatin remodelers and their significance in cancer and therapeutic resistance

v. Higher order cellular microRNA response

vi. Molecular mechanisms of the efficacy of fractionated radiotherapy

b. The role of long noncoding RNAs in skeletal 4-7 myogenesis

i. Further mechanistic characterization 4-8 of MUNC

ii. Does MUNC belong to a new class of 4-9 trans acting lncRNAs?

iii. Characterization of other lncRNAs 4-10 upregulated during myogenesis 
c. References

4-12

5. Appendix: Unpublished results: O-GlcNAc

misregulation andaneuploidy in cancer, and

the targeting of Cdc25a by miR-99a

a. Body

$5-1$

b. References

$5-11$

c. Figures

$5-12$ 


\begin{abstract}
:
It has become clear that proteins are not the sole regulatory molecules in the nucleus, as numerous functional RNAs are being identified and characterized. Noncoding RNAs are broken into two large classes: (1) short RNAs, typified by miRNAs, which bind and inhibit translation of mRNA; (2) long noncoding RNAs, a diverse class of molecules with a variety of regulatory roles. This dissertation examines two instances of ncRNA mediated regulation: firstly the role of microRNAs in the DNA damage response, and secondly, the role of long noncoding RNAs in skeletal myogenesis.
\end{abstract}

In our first study, we screened for microRNAs whose expression was increased in a radiosensitive breast cancer cells, and whose basal expression was higher as compared to radioresistant breast cancer cells. The miR-99 family was enriched in this population. We found transfection of miR-99 family members to sensitize radioresistant breast and prostate cancer cell lines to DNA damage. miR-99 transfection inhibited both homologous recombination and nonhomologous end joining DNA repair by reducing SNF2H expression. Finally we found that induction of miR-99 following DNA damage suppresses the induction of SNF2H after multiple rounds of DNA damage, resulting in decreased DNA repair. This suggests a molecular mechanism contributing to the efficacy of fractionated radiotherapy.

In our second study, we performed a bioinformatics screen to identify long noncoding RNAs upregulated during skeletal myogenesis. Using publicly available ChIP and RNA sequencing data, we found a set of putative lncRNAs upregulated during myogenic differentiation and validated them by qPCR. Among these hits was one lncRNA encoded in the MyoD Distal Regulatory Region, which we termed MyoD Upstream Non-Coding (MUNC). 
siRNA knockdown of MUNC significantly impaired myogenesis. MyoD recruitment to a number of its genomic targets was reduced by depleting MUNC, which itself associated with MyoD and these genomic loci. Finally, transfection of MUNC stimulated MyoD-dependent luciferase activity. These results suggest that MUNC can facilitate MyoD transcriptional activity, and contributes to MyoD expression through a feed forward loop. 


\section{Acknowledgements:}

\section{"Don't look at the dogs, work the lock!" -Magnum P.I.}

To my parents, Bill and Joanne: Thank you for giving me all the tools, opportunities and support you have over the years. I could not be who I am today without the sacrifices you made in the pursuit of my education and lessons you taught me in my upbringing. Thanks for being amazing role models, and teaching me that I can go after what ever I want, and do anything, as long as I throw myself into it with gusto and believe in myself. I love you both.

To my brothers, Noel, Shane and Conor: Thanks for excelling in each of your own ways, you crazy balls of talent. You're each an inspiration to me, keep it up.

To Kathleen Dodds: I'm sorry you couldn't be here to see this, you were a shining beacon in our family, and your example will last for generations. We had some good times didn't we? Yes, we did. Love you Nana.

To Glenn Doman: One of the greatest of men, a hero to thousands of children and parents, a rock of resolve with the kindest of hearts. You were one of my earliest inspirations and an exemplar of what it takes to change the world. Rest in peace knowing how many others take strength from your example.

To CdC: Know that you'll always have a place in my heart. All the best for you CB, always.

To my friends: Thanks for all the support down this long path. It wasn't easy, but it would have been much harder without the good times and support from each and every one of you. Backyard foos, pumpkin bowhunting over scotch, OBX shenanigans, rocking out, filing oeno, themox, William Atherton and Oompa Loompas, hookah in the papasan, things not to write in your dissertation, "dig man, there goes Mack the Knife", sipping in spandex by the side of the road, playing golf while getting your suit pressed, crushing rocks, following our hearts; it all helped me to get through this scary forest and come out the other end, better, stronger, faster and reasonably sane. Thanks folks, I'll be seeing you around.

To all my teachers, mentors and colleagues over the years: I would not be who I am, or where I am now without the lessons you imparted, and opportunities you have given me. A man stands on the shoulders of those who came before him, and I have many shoulders to be thankful for.

To my committee members James Larner, Marty Mayo, and Todd Stukenberg: Thanks for your attention and valuable advice through this process, I could always rely upon getting some unexpected insight and perspective in each of our meetings, and I appreciate your input into my scientific growth.

To my advisor and mentor Anindya Dutta: Thank you for seeing in me what you did all those years ago. Thank you for showing me the importance of curiosity, optimism, occasional self-delusion, drive and ambition. I respect you immensely as a scientist and a mentor, and I hope I can always come to you for counsel. I've enjoyed our conversations, and I hope to still. It's been a long and sometimes rocky path, as research is wont to be, but you've shown me what it takes to be a scientist, and I'll lean on the lessons I've learned under your tutelage and mentorship for the rest of my career.

Now on with the show... 


\section{Chapter 1}

\section{Introduction}

In the last half century since the discovery of the structure of DNA and the nature of the genetic code, the primary paradigm for gene expression has been that of a DNA blueprint encoding RNA messengers, which are then translated into functional proteins. This paradigm became strongly embedded into the collective conscious of molecular biology with the coining of the term "The Central Dogma of Molecular Biology" by Francis Crick(1), and has held true to a basic extent across all species. In the last decades however, cracks have been appearing in the concept of proteins as the sole functional machines in living organisms. With the release of the sequenced human genome, it became clear that only a small fraction of that DNA encoded proteins(2). However, a large proportion of the non-protein-coding majority of the genome is yet transcribed in a regulated, temporal and developmental context specific manner(3). Study of this transcribed pool of non-coding RNA is giving rise to numerous new classes of functional and regulatory RNA molecules, which appear in higher eukaryotes to have functions in numerous aspects of cellular metabolism and development $(4,5)$.

In retrospect, the concept of functional RNA should be hardly surprising, as one of the most basic and essential pieces of cellular machinery in all organisms, the ribosome, is composed largely of RNA, which is involved in both structural and mechanistic aspects of its function(6). The discovery of small-interfering RNAs and microRNAs introduced RNA as powerful regulators of development that could alter the expression of hundreds of targets, and hold equal footing with transcription factors as powerful controllers of gene expression(7). Still, the 
functional categories of the majority of the transcribed genome are yet difficult to predict and have poor sequence conservation, indicating either that a high level of transcriptional noise is present in the cell, or more likely that there exist numerous uncharacterized classes of noncoding RNA to be discovered.

A rapidly emerging diverse class of non-coding RNAs has been termed long non-coding RNAs or lncRNAs. Defined as RNAs over 200 bases with low protein coding potential, these RNAs have been found to be involved in epigenetic silencing, regulation of splicing and regulation of mRNAs at the levels of transcription and mRNA stability(4). These RNAs have intermediate conservation between protein coding sequences and untranscribed regions, but are integral to a number of cellular and developmental processes. Intersections of ChIP-sequencing and RNA-sequencing studies have found thousands of independently regulated ncRNAs present and expressed in both mice and humans(8-10). Because of poor sequence conservation, prediction of function of these molecules is difficult, but new tools allow their genetic manipulation in vitro and in vivo, discovery of protein binding partners and genomic localization, and hopefully eventual grouping into distinct mechanistic categories. This chapter will introduce the research completed for this dissertation, and review the role of non-coding RNAs in two cellular contexts: first, the various roles of microRNAs in regulating the DNA damage response, and second, roles of long noncoding RNAs in regulating gene expression during development and disease.

\section{A. Dissertation Overview}

In this dissertation I will show two examples of noncoding RNA mediated regulation of gene expression in disease and development. In both of these studies we utilized global screens 
to identify members of specific classes of noncoding RNAs whose expression changed in response to either extracellular stimulus in the form of DNA damage in the first study, and during the course of skeletal myogenesis in the second. In both cases we found noncoding RNAs whose differential expression conferred strong phenotypes by regulating processes essential in their respective cellular context: firstly, DNA double strand break repair in response to ionizing radiation, and secondly, the transcriptional cascade of skeletal myogenesis.

\section{B. microRNA mediated regulation of the DNA damage response and the implications of such regulation for cancer.}

MicroRNAs are a class of small 17-25 nucleotide RNA molecules that regulate gene expression in higher eukaryotes. Originally discovered in C. elegans(11), thousands have been discovered across metazoans, to regulate diverse cellular and developmental processes, as well as contributing to genetic diseases such as cancer(12, 13). MicroRNAs have been discovered that behave like classical oncogenes and tumor suppressors, regulating expression of numerous gene targets involved in cell proliferation, cell cycle checkpoints and DNA repair. MicroRNA regulation contributes to cell fate decisions by adjusting the contribution of tumor suppressors and proto-oncogenes to various extracellular and intracellular stimuli. This review will examine microRNAs that regulate the DNA damage response to double strand breaks, subsequent checkpoint activation and radiation sensitivity in cancer.

\section{i. Mechanisms of double strand break repair}

The cellular response to DNA damage involves several mechanisms to sense and repair various types of damage, and depending on the cell type, the extent of the damage incurred, and the stage of the cell cycle, allow cells to decide whether to survive or initiate apoptosis. Double 
strand breaks, being one of the most severe types of DNA damage, are repaired by multiple mechanisms, and involve multiple damage sensing kinases that initiate a cascade of phosphorylation and ubiquitination leading to checkpoint activation and repair. The Ataxia Telangiectasia Mutated (ATM) and DNA-PKcs kinases both direct double strand break repair and checkpoint activation through homologous recombination and non-homologous end joining respectively. ATM senses double-strand breaks and acts in conjunction with its homolog ATR, which senses single stranded DNA generated by processing of DSBs or stalled replication forks $(14,15)$. ATM and ATR are recruited to sites of DNA damage by the Mre11-Rad50-NBS1 complex, phosphorylating histone $\mathrm{H} 2 \mathrm{AX}$ at these sites, as well as the Chk1 and Chk2 checkpoint proteins, which can lead to p53 activation and cell cycle arrest or apoptosis. Through interactions with ubiquitinated histones, BRCA1 is also recruited to these sites and phosphorylated by ATM, colocalizing with numerous interaction partners to facilitate homologous recombination by BRCA2 and Rad51 and to facilitate checkpoint activation(16). This signaling cascade and subsequent repair requires the activity of multiple kinases and ubiquitinases as well as large chromatin remodeling complexes, (17-19). Downregulation of any of these components could mimic their mutation in impairing DSB repair and lead to a cancer phenotype, as seen in BRCA1 and BRCA2 mutation.

In addition to homologous recombination, DSBs are also repaired by a parallel mechanism of non-homologous end joining. NHEJ is the predominant mechanism of DNA repair in G1 cells in higher eukaryotes, and involves ligation of free double stranded DNA ends in response to DSBs. Free DNA ends are sensed by the Ku70/80 proteins, which then recruit the ATM/ATR homolog DNA-PKcs kinase, which cooperates to tether these DNA ends. A complex of XRCC4 and DNA Ligase IV then are recruited to ligate the DNA, repairing the break(20). 
Analogous to HR repair, recruitment of these factors also requires the action of chromatin remodeling complexes(21), which are increasingly being found as targets of microRNA regulation(22-24).

\section{ii. MicroRNA biosynthesis and regulatory action}

MicroRNAs are typically encoded within long ncRNA transcripts called primary microRNAs that often encode several microRNA genes. These long transcripts are cleaved into approximately 70nt precursor microRNAs by the Drosha/DGCR 8 complex before being exported into the cytoplasm. These hairpin containing precursors are then cleaved by Dicer into 17-25nt long dsRNA, whose individual strands associate with Argonaute proteins. These complexes bind degenerate complementary sequences in the 3'UTRs of target mRNAs, resulting in translational inhibition or degradation of these targets $(7,25)$. Since the initial discovery of microRNAs, over 1000 have been discovered in humans, with similar numbers in mice and other mammals. With the ability to inhibit multiple mRNAs per microRNA, microRNAs exert extensive post-transcriptional regulation that can fine tune expression of thousands of genes in a context specific manner. Like proto-oncogene and tumor suppressor proteins, microRNA expression is altered in multiple cancer types, resulting in a dramatically altered ability to sustain and repair DNA damage, and withstand checkpoint activation(17).

\section{iii. Regulation of microRNA expression during the DNA damage response}

A number of microRNAs have been discovered to change in expression following DNA damage. Several important checkpoint proteins, including p53, regulate microRNA expression $(26,27)$, and inversely microRNAs exert post-translational control over some of these same checkpoint proteins. MicroRNAs regulated following DNA damage, particularly if they are 
also unregulated or misregulated in cancers, will help understand and exploit mechanisms by which cancers avoid cell death when treated with radiation or cytotoxic chemotherapy.

\section{iv. MicroRNAs upregulated following DNA damage}

One of the earlier discovered examples of a microRNA regulated by the DNA damage response is that of miR-34a, which was found to be a direct transcriptional target of p53 following DNA damage, and could induce cell cycle arrest by downregulating Cyclin E2, CDK4 and the hepatocyte growth factor c-MET(28). Intriguingly, miR-34a completes a positive feedback loop with p53 by downregulating SIRT1, a deacetylase which inhibits p53 activity, thus enhancing p53 dependent cellular response to DNA damage(27). The oncogenic microRNA miR-21 is upregulated in numerous cell lines after DNA damage (29-31). miR-21 was originally found to be upregulated in a number of glioblastoma lines functioning in an antiapoptotic role(32), and has since been found to be upregulated in numerous cancers(33-36). Similarly, the miR-17-92 oncomiR cluster is upregulated following $\operatorname{DSBs}(30,31,37)$. In contrast to miR-34a, the upregulation of miR-21 and the miR-17-92 cluster following irradiation suggests a strategy by which cancer cells evade cell death following DNA damage by upregulating pro-survival microRNAs.

\section{v. MicroRNAs downregulated following DNA damage}

A few members of the let-7 family of microRNAs have been consistently found to be downregulated following DNA damage. Expression of Let-7a and b was found to be suppressed in normal human fibroblasts 1 hour after being treated with ionizing radiation or etoposide(29). Another study found all of the members of the let-7 family except let-7g to be downregulated 2 hours following irradiation of normal lung epithelial cells as well as lung cancer cells(38). This is 
significant as the let-7 family is generally tumor suppressive, targeting the RAS oncogene(39), and lower expression of let-7 is a predictor of poor outcome in lung cancers(40). Forced expression of let-7 was able to sensitize lung cancer cells to DNA damage, suggesting this loss in expression was a method by which cancers could evade cell death following DNA damage.

\section{vi. MicroRNAs with variable expression following DNA damage}

Somewhat confusingly, most DNA damage responsive microRNAs have been found to have increased or decreased expression depending on the cell type and DNA damaging agent (41). In a screen for microRNAs whose expression changes following DNA damage in fibroblasts, let-7d, e, g and i were found to be upregulated an hour following exposure to ionizing radiation(29), whereas let-7a and $b$ were found to be downregulated. In fact, in this same study, let- $7 \mathrm{~b}$ was found to also have decreased expression following etoposide treatment, but increased expression following exposure to hydrogen peroxide. Several other groups of microRNAs, including the miR 16 family(42), miR-26b and miR-99 family can be both upregulated or downregulated depending on cell type or mode of inducing DNA damage(29, 31, $38,43-45)$. It remains important to parse the mechanisms that drive the regulation of these various microRNAs in response to DNA damage, and well as the reasons for such variation between cell types and DNA damage modality. Subtyping cancers by their microRNA expression profile in response to DNA damaging cytotoxic agents should be useful to predict the therapeutic response of cancers as well as determine the chemotherapy regimen best suited for an individual patient. 


\section{vii. MicroRNAs targeting DNA damage sensing and repair proteins}

Of equal import to determining which microRNAs are differentially regulated by DNA damage, is identifying components of the DNA damage response machinery that are targeted by these microRNAs. MicroRNAs target components of the DNA repair pathway involved in sensing and repairing DNA damage, potentially stimulating apoptosis in response to DNA damage in non-essential cells, but perhaps also leading to mutations in cells with faulty checkpoint mechanisms. Recently, the miR-182-5p, a microRNA enriched in some breast cancers, was found to target several components of the HR repair pathway, including BRCA1, 53BP1 and Rad17, suggesting misregulation of this microRNA can potentially phenocopy BRCA1 mutation. Overexpression of miR-182-5p sensitized cells to PARP inhibition, which is similarly seen in BRCA1 mutant cells. This result suggests that cancers over-expressing miR182-5p may also be effectively targeted by PARP-inhibitors $(46,47)$. The ATM kinase itself is targeted by numerous microRNAs, including miR-18a, miR100, miR-101, miR-181 and miR421(48-52).. Targeting of ATM leads to DNA repair defects as well as loss of cell cycle checkpoints in response to DNA damage. While this may contribute to spontaneous cancers, it should also be exploitable therapeutically, as mutations or misregulation of these microRNAs lead to ATM defects that result in exquisite sensitivity of these cells to DNA damaging radiation and chemotherapy. Interestingly, miR-101 can also target DNA-PKcs, suggesting that it can suppress both pathways of double strand break repair, and highlighting the significance of misregulation of miR-101 in cancers $(50)$.

Terminally differentiated non-dividing cells have a reduced need for efficient DNA repair, and thus often have reduced expression of DNA repair proteins needed for cycling cells, particularly involved in double strand break repair $(53,54)$. One such example is that of miR-24. 
miR-24 was found to target a fundamental component of the DNA damage signaling pathway, $\mathrm{H} 2 \mathrm{AX}$, in terminally differentiated cells(55). miR-24 is upregulated in mature blood cells, and reduces the capability of these cells to repair double stranded breaks, presumably through impaired recruitment of repair factors to sites of DNA damage by phosphorylated H2AX. When introduced into K562 cells in culture, miR-24 reduced their capacity for DNA repair, resulting in genomic instability and radiation sensitivity. In contrast, and highlighting a potential mechanism of radioresistance in cancer, downregulation of miR-24 in these same cells enhanced their ability to repair double strand breaks and survive treatment with bleomycin.

\section{viii. microRNAs targeting checkpoint activation proteins}

A number of cell cycle proteins that are targets of checkpoint mechanisms and induced by DNA damage are also targets of microRNAs, and the misregulation of these targets contribute to cancer development and sensitivity to DNA damaging therapies. Cdc25a, a key component of the intra-S-phase DNA damage checkpoint, is a target of numerous microRNAs, most notably the let-7 family(Johnson et al., 2007), miR-15a(56), miR-16(57) and miR-21(58). Cdc25a is a phosphatase that is responsible for removing inhibitory phosphorylation of CDK2. Cdc25a is targeted for degradation by the checkpoint transducer kinases Chk1 and Chk2 after DNA damage during S-phase to stop DNA replication and to allow DNA repair (59). This acute and rapid degradation of Cdc25a is facilitated by the titration of steady state levels of Cdc25a by microRNAs to lower the threshold of checkpoint activation that leads to intra-S phase cell cycle arrest. Similarly, miR-16 exerts posttranscriptional regulation of Cdc25a to enable faster changes in gene expression following DNA damage (57). 
As well as regulating the expression of numerous microRNAs, p53 its self is a target of a number of microRNAs. miR125b was found to target p53(60), as well as other members of its downstream transcriptional network(61), suggesting a pro-survival function. This observation is partially borne out by its overexpression in colorectal cancer(62) as well as its ability to stimulate development of B-cell malignancies in mice(63), however is contradicted by a study that found it to be tumor suppressive in the context of breast cancer(64). In a screen for microRNAs targeting p53, miR-504 was found to inhibit p53 translation through two target sequences in its 3' UTR, and its ectopic expression could decrease p53 expression in cell culture, as well as stimulate tumor growth in mouse xenografts(65). In addition, p53 has been found to be regulated by miR$380,33,1285,30 \mathrm{~d}$ and 25 , resulting in a suppression of its downstream effects, further reviewed in (66).

\section{ix. microRNAs on the frontier of cancer diagnosis and treatment}

It has become clear that microRNAs are an essential mode of regulating gene expression in higher eukaryotes, on equal footing with transcriptional and post-translational control. Misregulation of microRNA expression is frequently seen in cancer, and can mimic mutations to known tumor suppressors, often in DNA repair pathways. Categorization of cancers by microRNA expression, and their targets, should allow more effective grading and prediction of therapeutic response, as well as tailoring of treatments to individual subtypes. Examining microRNA expression within the context of the global genome and proteome characterization among large groups of patients should present hope for more effective personalized treatment of cancer. 


\section{The role of Long Non-coding RNAs in regulating gene expression}

Initial studies characterizing long non-coding (lnc) RNAs in the mammalian transcriptome left open the possibility that the expression of these sequences was a consequence of "transcriptional noise" or promiscuous RNA polymerase II activity. Many of these putative non-coding sequences lie in regions proximal to the promoters of known coding genes, or as antisense transcripts to coding genes. However, more recently a large group of expressed sequences were found to have their own independent regulation characterized by histone modifications and splicing signals, with very low protein coding potential, located in genomic regions distant from known protein coding genes(4). Studies building genomic maps of long intervening non-coding RNAs found over 1000 of these lincRNAs in humans and mice. These noncoding genes had histone $\mathrm{H} 3$ lysine 4 trimethylation marking transcriptional start sites, lysine 36 trimethylation indicating actively transcribed regions, as well as exon-junctions in the RNA sequencome, indicating splicing was occurring. In addition, most of these sequences showed evolutionary conservation above that of non-expressed sequences and many were found to be regulated by a number of important developmental and homeostatic transcription factors $(8,9)$.

Without rules for prediction of the function of these non-coding genes, it remains difficult to classify them into categories beyond their genomic locations and expression patterns. In addition, we do not understand the mechanisms of evolution of these lncRNAs when removed from the constraints of the amino acid codon based genetic code. Functional ncRNAs may sustain higher mutation rates while maintaining their function, as compensatory mutations may occur more easily to preserve their secondary structure. Better characterization of functional lncRNAs will provide insight into the nature of their domains and interaction characteristics, allow more effective prediction algorithms, and a better understanding of functional homology 
between species despite the weaker sequence homology. Below I describe several examples of how novel lncRNA regulate gene expression, to highlight their diverse modes of action and significance in disease and development. A number of these examples are illustrated in Figure 1.

\section{i. Xist mediated silencing of the inactive $\mathrm{X}$-chromosome}

One of the earlier discovered examples of gene regulation by a long non-coding RNA is that of Xist in X-chromosome inactivation. In females, the majority of genes contained on one of the two X-chromosomes in each cell are silenced, accounting for the similar expression of these genes between females and males, who only possess a single X-chromosome. During female

development, the non-coding RNA Xist is transcribed from the X-chromosome that is destined to become inactivated in each cell(67). Xist associates with the regions of the chromosome that are to be silenced, resulting in the formation of "Xist clouds", recruitment of Polycomb Group Repressive complexes and addition of repressive chromatin marks(68, 69). Another ncRNA Tsix is produced from the Xist gene in the antisense direction on the active $\mathrm{X}$ chromosome, which antagonizes Xist, preventing inactivation of the active $\mathrm{X}$ chromosome(70). Interestingly, though Xist seems to be essential for X-inactivation from mice to humans, its sequence conservation is very poor, which may further suggest we need new bioinformatic tools and algorithms to predict IncRNA function based upon secondary structure rather than sequence conservation. Xist and Tsix regulation of $\mathrm{X}$-inactivation is further reviewed in(71).

\section{ii. Pro-metastatic IncRNA HOTAIR and PRC recruitment}

A lncRNA encoded within the HOX gene cluster was recently discovered to promote metastasis in breast cancers, and its expression had a negative effect on patient prognosis. Termed HOTAIR, this lncRNA was highly overexpressed in metastatic breast cancers, and 
increased invasiveness when overexpressed in breast cancer cells in culture. Overexpression of HOTAIR altered the occupancy of PRC2 across the genome, rearranging the landscape of repressive histone $\mathrm{H} 3 \mathrm{~K} 27$ trimethylation in cells, making them take on a more fibroblastic phenotype. HOTAIR was shown to act in trans to alter the target specificity of these repressive polycomb group complexes, having a dramatic effect on gene expression, repressing antimetastatic genes in cancer(72). In line with these results, HOTAIR was found to be a transcriptional target of the estrogen receptor in breast cancer cells, suggesting a role in their hormone responsiveness(73). Interestingly, HOTAIR was also found to be overexpressed and have prognostic significance in hepatocellular and nasopharyngeal carcinomas(74-76), suggesting a widespread role in tumor aggressiveness, and powerful efficacy in driving chromatin rearrangement and promoting metastasis in multiple tissue lineages. Interestingly, in contrast to Xist, HOTAIR seems to have very little effect in mouse cells, suggesting its functional significance was a recent evolutionary event(77).

\section{iii. IncRNAs with cis enhancer-like functions}

In addition to having roles in epigenetic suppression of gene expression, it is becoming clear that certain lncRNAs are important for driving transcription of protein coding genes. A number of groups have found an abundance of transcription originating from enhancer regions of protein coding genes in mammalian cells, suggesting a possible lncRNA role in cis enhancer activity(78-81). In some of these cases, depletion of an enhancer-encoded lncRNA decreased expression of the protein coding gene driven by the enhancerr. In addition, transcription of the lncRNAs at these enhancers was stimulated by conditions known to increase expression of the enhancer-driven gene. Several examples of this class of lncRNA have been found to be important for development of tissues in mammals. The expression of the bHLH transcription 
factor Neurogenin 1 was dependent on the expression of a lncRNA located $7 \mathrm{~kb}$ upstream of the Neurogenin 1 transcriptional start site. Polycomb group proteins, suppress the expression of both the lncRNA and neurogenin1 itself, suggesting that the repression of neurogenin 1 was in fact mediated by the repression of the $\operatorname{lncRNA}(82)$.

One of these enhancer elements was also found to be involved in brachydactyly type $\mathrm{E}$ when its translocation resulted in disruption of a cis- regulatory element (CRE) encoding a lncRNA. This lncRNA-encoding CRE regulated the expression of parathyroid like-hormone (PTHLH) and the sex determination gene SOX9. This regulation occurred through intrachromosomal looping bringing the CRE close to the proximally located PTHLH gene, and interchromosomal physical associations between the CRE and the SOX9 gene. Disruption of this lncRNA, as well as its translocation, reduced expression of both these genes (83). A group of these cis-acting enhancer-like lncRNAs, termed ncRNAa for noncoding RNA activating, acts through recruitment of the transcriptional co-activator Mediator. Mediator physically interacts with a number of these lncRNAs, and depletion of these lncRNAs or Mediator decreased expression of adjacent target genes. This interaction was found to facilitate chromatin looping of the adjacent genes, leading to transcriptional activation and their expression(84). These examples suggest that many enhancer-encoded lncRNA in the mammalian genome are essential to regulate the cis-activating function of the corresponding enhancer, in particular to change the looping of the DNA so as to bring the target genes in proximity to protein factors required for transcription. 


\section{iv. Trans-acting enhancer IncRNAs}

A few examples have recently been uncovered of lncRNAs acting to regulate gene expression in trans, in some cases still acting upon adjacent genes present in cis on the genome. A large spliced lncRNA termed NEST, for nettoie Salmonella pas Theiler's (translated: cleanup Salmonella not Theiler's), is encoded proximally and antisense to the Interferon gamma(IFNG) locus in mice and humans(85). In fact in humans, this antisense transcript actually overlaps the IFNG and IL-26 genes. This lncRNA was responsible for the unusual phenotype of mice being unable to clear infection by Theiler's murine encephalitis, while being more resistant to lethal Salmonella enterica infection. Expression in trans increased IFN- $\gamma$ produced by activated T-cells, and resistance to Salmonella infection. These phenotypes were a result of NeST recruiting the histone methylase MLL to the IFNG locus, increasing expression of IFN- $\gamma$ in trans and altering microbial susceptibility.

A fascinating case of trans-acting RNA mediated transcriptional regulation is that of the Steroid Receptor Activator SRA gene, which encodes both a protein (SRAP) and a functional lncRNA (SRA) that act as coregulators of nuclear receptor transcriptional activity(86). In addition to nuclear receptors, SRA facilitates the transcriptional activity of p53(87) as well as MyoD(88) in different cellular and developmental contexts. Overexpression of SRA RNA, but not SRA protein(SRAP), facilitated skeletal muscle transdifferentiation of mouse fibroblasts with forced MyoD expression. SRAP actually inhibits muscle differentiation by binding SRA RNA and preventing it from activating $\operatorname{MyoD}(89)$, a striking example of a gene encoding two products with opposing effects on transcription through production of a lncRNA. 


\section{v. MALAT1 regulation of gene splicing}

LncRNAs regulating gene expression through alternative splicing or mRNA stability have been recently discovered, such as Metastasis Associated Lung Adenocarcinoma Transcript 1 (MALAT1). MALAT1 is overexpressed in a number of metastatic cancers(90-92). MALAT1 is also overexpressed as the result of chromosomal rearrangements giving rise to specific tumor types, notably undifferentiated embryonal sarcoma of the liver and renal cell carcinoma $(93,94)$. In contrast to HOTAIR and Xist, MALAT1 regulates a large set of genes by recruiting Serine Arginine alternative splicing factors to transcription sites. This leads to suppression of p53 activation as well as promoting expression of the oncogenic transcription factor B-Myb, driving G1/S as well as mitotic progression(95). In addition to promoting metastasis, MALAT1 modulates synaptic density in cultured neurons(96). MALAT1 is notable along with another lncRNA Multiple Endocrine Neoplasia-beta(MEN-beta) and Kaposi’s sarcoma-assoicated herpesvirus polyadenlated nuclear long-noncoding RNA(PAN), for containing a triple helical structure in its 3' end which significantly stabilizes the RNA and promotes its nuclear localization. It is intriguing that some cellular lncRNAs develop, and some viruses mimic, novel mechanisms to avoid the many RNA decay enzymes in higher eukaryotes $(97,98)$.

Interestingly, recent studies suggest that MALAT1, while highly conserved for a lncRNA, is not required for embryonic mouse development. Its knockout does alter the expression of a specific subset of genes in an adult mouse. Thus either MALAT1 has limited importance during development, despite a significant role in cancer progression, or recent evolution has increased the role of MALAT1 in human cells $(99,100)$. A third potential explanation of this observation is that MALAT1 plays a role in adult cell regeneration in response to injury, which is supported by a recent study which identified MALAT1 as a target of the repressor of skeletal muscle 
development myostatin in an adult skeletal muscle regeneration model. MALAT1 is upregulated during skeletal muscle differentiation, and siRNA knockdown in culture diminishes proliferation of adult derived myoblasts(101). This could suggest a greater role of MALAT1 in the expansion of adult stem cells, which may be hijacked to promote proliferation and metastasis during cancer progression.

\section{vi. IncRNA mediated suppression of microRNA mediated mRNA decay}

microRNAs act by binding to degenerate complementary sequences in mRNA, typically within their 3' UTRs(7). MicroRNAs themselves are typically transcribed as long noncoding RNAs called primary microRNAs, which are then processed into short RNA fragments which regulate translation and mRNA stability. Transfection of cells with vectors encoding exogenous transcripts containing multiple copies of a microRNA target sequence to titrate microRNAs away from their endogenous targets is an experimental method, termed microRNA sponge, that has been utilized in the last several years to inhibit their action(102). Intriguingly, it turns out this mechanism is also found in nature, as certain endogenous lncRNAs have been discovered to titrate away microRNAs, allowing for the translation of their targets. This competitive inhibition of microRNAs has been seen in lncRNAs as well as the 3'UTR of protein coding genes, and represents a hybrid class of functional regulatory RNAs that has been referred to as competing endogenous RNA(ceRNA)(103).

One of these long noncoding ceRNAs was discovered to play a significant role in skeletal muscle development by titrating away miR-133 from its targets MAML and MEF2C, both important pro-myogenic transcription factors that are induced during the differentiation program. This lincRNA, linc-MD1 when overexpressed caused differentiation to proceed more rapidly, 
and conversely differentiation was delayed upon knockdown of linc-MD1. Of potential significance to muscular wasting diseases, linc-MD1 was decreased in muscle cells from individuals with Duchenne muscular dystrophy(104). Lnc-ROR is another recent example of a lnc-ceRNAs playing an important developmental role, in that it regulates human embryonic stem cell renewal, preserving Oct4, Sox 2 and Nanog expression by titrating away mature miR-145 and preventing the repression of these core transcription factors essential for stem cell function(105).

Optimal secondary structures for efficient microRNA titration designed into newer generation artificial sponges called tough decoys(106) was the incorporation of a near-circular region of single stranded RNA containing microRNA target sites. In a fascinating parallel, this design has now been discovered in nature in the form of natural circular transcripts, termed circRNAs(107). Thousands of these transcripts have been recently discovered, though few experimentally validated to titrate microRNAs. One such case is that of ciRS-7, a circRNA expressed in the human and mouse brain which contains over 70 putative microRNA target sites, 63 specific for miR-7. Overexpression of ciRS-7 impaired brain development in zebrafish, a phenotype seen with miR-7 knockdown. Many of these circRNAs seem to form by head to tail splicing of antisense transcripts to protein coding exons, forming new ncRNA transcripts that can regulate expression of their overlapping protein coding genes by preventing the latter's repression by microRNAs $(108,109)$.

\section{vii. Challenges of future study of IncRNAs in higher eukaryotes}

Although still a very new field of study, it is becoming rapidly clear that one of the major causes of phenotypic complexity in higher eukaryotes relative to lower eukaryotes is the level of 
involvement of long noncoding RNAs in regulation of gene expression. While the number of protein coding genes between vastly different organisms often varies to a surprisingly small degree, these examples of IncRNA mediated regulation of gene expression and function suggest that the complexity could originate in these functional lncRNAs. Much effort should be focused on prediction of the secondary structure, binding partners and new paradigms of evolutionary conservation of these molecules. The number of different mechanisms and binding partners suggest that the term noncoding RNA is far too broad for such a diverse set of functional molecules, and hopefully they will eventually be organized into subcategories of characterized and predictable function. There remains much to discover regarding the importance of these diverse components of the cellular machinery. Hopefully this discovery will also lead to new diagnostic and treatment tools as they bring more clarity to our understanding of regulation of gene expression in multiple developmental and disease contexts.

\section{Regulation of skeletal muscle development}

Like most developmental processes, skeletal muscle development occurs through an ordered transcriptional cascade, with multiple feedback loops regulating the order and timing of gene expression as cells proceed down their lineage pathway. The cells that give rise to muscle originate in the somites of the early embryo, which gives rise to the dermatomyotome. These cells in the dermatomyotome express the paired homeobox transcription factors Pax3 and Pax7, which are crucial for maintaining the proliferative state of these embryonic myoblast precursors, as well as maintaining the pool of adult satellite muscle stem cells(110). 


\section{i. The genetics of skeletal myogenesis}

The cascade of differentiation of these muscle stem cells into mature muscle fibers is driven by a family of basic helix loop helix (bHLH) transcription factors called muscle regulatory factors (MRFs). The first to be discovered of the MRFs was MyoD, which would prove a model for much study of transcription factor function in development. MyoD was found to be a master regulator of myogenesis in that its forced expression could trigger a reprogramming cascade that caused fibroblasts as well as other cell types to transdifferentiate into skeletal muscle $(111,112)$. After this discovery, a number of closely related proteins, namely Myf5, Myogenin and MRF4 were discovered which also had similarly important roles in orchestrating muscle differentiation(113-115). The MRF proteins all share a consensus DNA target sequence of CANNTG called an E-Box, several copies of which are usually found near the promoters of MRF transcriptional targets(116). MyoD and My5 are very closely related proteins that have overlapping roles in dictating myogenic cell fate. Considering its striking ability to induce myogenesis, it was initially surprising that knockout of MyoD had little effect on skeletal muscle development during embryogenesis in mice. In fact, Myf5 and MyoD are redundant during development(117), and only a double knockout will significantly impair skeletal muscle development during embryogenesis(118). However, MyoD-/- adult mice are significantly impaired in their ability to regenerate skeletal muscle after injury, indicating that while MyoD may be redundant during embryogenesis, it is necessary for initiating differentiation in adult satellite stem cells(119).

In contrast to Myf5 and MyoD, Myogenin is indispensible to myogenesis and its knockout results in neonatal lethality(115). Myogenin is a downstream target of Myf5 and MyoD, and also acts in concert with the other MRFs to activate other target genes. However, myogenin 
expression alone is not sufficient for efficient myogenesis(120), and it requires the presence of MyoD or Myf5 to fully activate its targets. Expression and subsequent activity of myogenin requires widespread epigenetic remodeling in myoblasts by MyoD and its associated histone modification complexes. Myogenin itself more directly recruits transcriptional activators to these regulatory regions following $\mathrm{MyoD}$ mediated epigenetic reprogramming. MRF4 is interesting in that its role is less well defined, but has characteristics of both Myf5 and MyoD on one hand and Myogenin on the other. Forcing expression of MRF4 can rescue the defect seen with Myf5 -/-, MyoD-/- double knockout mice(121), and expressing MRF4 under control of the myogenin promoter also can rescue the defect seen in myogenin knockout, indicating that MRF4 has overlapping roles with all three other members of the MRF family(122).

The MRFs have numerous binding partners that facilitate their ability to bind DNA and activate transcription, and in fact some of these partners confer dual functionality depending on the cellular developmental context. All of the MRFs bind to coactivators called E-proteins, forming heterodimers that then have the ability to bind to the E-box consensus sequence on DNA(123). Inhibitor of differentiation (ID) proteins, transcriptional targets of the Pax genes(124), prevent initiation of the differentiation cascade by MRFs in satellite cells by competing for E-proteins(125), and thus preventing the formation of the MRF/E-Protein heterodimers in MyoD expressing, Pax3 positive satellite cells.

In addition to E-proteins, MRFs, particularly MyoD, directly interact with the Mef2 family of transcription factors. Mef2 proteins have their own DNA consensus sequence, often found in proximity to E-boxes in the promoter regions of myogenically activated genes, and cooperate with MRFs to regulate gene expression(Molkentin et al., 1995). Mef2a, Mef2c and Mef2d are all expressed in skeletal muscle, and knockouts of individual genes (skeletal muscle- 
specific knockout for Mef2c) do not impair skeletal muscle function suggesting overlapping roles for the Mef2 proteins. Mef2c knockout causes embryonic lethality due to cardiac defects, suggesting that the Mef2 proteins have varying importance for skeletal and cardiac myogenesis $(126,127)$. Mef2c is itself a target of MyoD activation, and the timing of its expression may be important for the proper temporal regulation of myogenesis, particularly in satellite cells, where stimuli from injury or extracellular signals promote the entry of quiescent satellite cells into the myogenic differentiation cascade. Recently it was reported that Mef2c was part of a negative feedback circuit with Notch3, where Notch3 inhibits expression of Mef2c, delaying its rise in early myoblast differentiation. Later upregulation of Mef2c by MyoD leads to the expression of microRNAs miR-1 and miR-206, which target Notch3 and allow terminal differentiation (128).

MyoD recruits the histone acetyl transferase proteins p300 and PCAF, to activate the transcription of MyoD target genes (129). In proliferating myoblasts HDAC1 is associated with MyoD on chromatin preventing the association of the latter with PCAF and thus preventing target gene activation(130). The interaction of HDAC1 with MyoD suggest that MyoD may have a repressive role in proliferating myoblasts at certain loci, which switches to activation when the HDAC1 is displaced by a HAT upon initiation of differentiation. Subsequently, widespread chromatin remodeling occurs through the recruitment of SWI/SNF chromatin remodeling complexes by MyoD to its target genes. This seems to occur in a temporally distinct manner, with the HAT complexes PCAF and p300 being brought to chromatin by MyoD to acetylate regions of activation, followed by recruitment of the SWI/SNF ATPase Brg1, which facilitates remodeling of these promoter regions(131). 


\section{ii. MicroRNA regulation of skeletal muscle development}

As hinted before, in addition to directly activating transcriptional targets which positively regulate differentation, numerous microRNAs are targets of MyoD and its family members, which then inhibit stem cell maintenance factors, positive regulators of cell proliferation and inhibitors of differentiation. In particular, the microRNAs miR-1 and miR-206 are highly expressed in skeletal muscle, and are rapidly induced following induction of myogenesis in myoblasts and satellite cells. A number of other microRNAs are also induced during myoblast differentiation, including mir-133, 26a, 378, 486, and 762(reviewed in (132)). These microRNAs mediate MyoD dependent downregulation of Pax 3 and Pax7 proteins, cell cycle exit by repression of Akt signaling, facilitation of MyoD activation by repression of MyoR, a MyoD inhibitory factor, as well as induction of Mef2C expression by repression of HDAC4. Strikingly, overexpression of most of these microRNAs stimulate myoblasts to differentiate into myotubes, indicating once lineage commitment occurs, microRNAs become powerful drivers of terminal differentiation.

\section{iii. The role of long noncoding RNAs in skeletal muscle development}

In addition to microRNAs, it is becoming clear that long noncoding RNAs are heavily involved in the regulation of myogenesis. A few recent studies have identified ncRNAs upregulated during muscle differentiation, and playing important roles in regulating $\mathrm{MyoD}$ expression and activity. IncRNAs have been found to overlap a number of MyoD binding sites across the genome, and be transcribed in a MyoD dependent manner(133). These lncRNA transcribing MyoD binding sites are enriched in the enhancer regions of MyoD target genes involved in myogenesis, and likely play a role in facilitating this enhancer activity, as in the case 
of other lncRNAs that facilitate chromatin looping and recruitment of Mediator to transcriptional start sites(84). LncRNAs can also contribute to regulation of MyoD expression. MyoD has also been shown to regulate the lncRNA H19, located at the Igf2 locus. Igf2 can interact with MyoD in vitro, and also indirectly inhibit its expression. H19 expression on the other hand represses transcription of Igf2, forming a negative feedback loop whereby MyoD derepresses its own expression by inhibiting Igf2 through H19 expression(134). There are several isoforms of myosin heavy chain (MHC), which are differentially expressed during late myogenesis depending on the fiber type of the skeletal muscle being formed. Embryonic MHC is expressed in myotubes prior to expression of these various MHC isoforms. Recently a long noncoding natural antisense RNA at the MHC IIb locus in rats, the predominant isoform in adult rats, was found to suppress MHC IIb transcription in favor of embryonic, and likely represents a mechanism by to regulate expression of the proper MHC isoform temporally during development (135).

Some of the lncRNAs involved in cancer metastasis also play a role in skeletal myogenesis. The lncRNA MALAT1 was recently implicated in the control of skeletal muscle differentiation, and is antagonized by the inhibitor of myogenesis myostatin. Myostatin is a potent inhibitor of both myoblast proliferation and differentiation. MALAT1 is expressed in proliferating myoblasts, and when treated with myostatin, its expression is reduced. Depletion of MALAT1 in myoblasts suppressed myoblast proliferation with a G0/G1 arrest. Additionally, MALAT1 levels increase 4-7 fold during differentiation, and MALAT1 depletion decreases myogenin induction during differentiation (101). The Steroid Receptor RNA Activator, and its protein isoform SRAP have opposite roles in facilitating MyoD activity. SRA binds to MyoD and facilitates its activation of responsive promoters. SRAP can inhibit this co-activation of 
MyoD by binding to SRA directly through one of its secondary structural hairpin domains. The ratio of SRA to SRAP increases during myogenesis, which rescues SRA from repression by SRAP and allows SRA to act as a co-activator of $\operatorname{MyoD}(89)$.

While much has been learned about the regulation of skeletal myogenesis since the discovery of the MRFs over two decades ago, there remains much to be elucidated, particularly at the new frontier of regulation by long noncoding RNAs. Skeletal muscle differentiation is a powerful model system with strong implications in developmental biology as well as diseases such as muscular dystrophies which are characterized by failure to differentiate and exhaustion of the stem cell pool. Thus discovering new roles for long noncoding RNAs in skeletal myogenesis should provide a broader insight into their roles in development in general, as well as explore the use of IncRNAs as targets for new approaches in the burgeoning field of regenerative medicine.

\section{E. Goals of Current Research:}

\section{i. The role of microRNAs in the DNA damage response:}

Goal 1: Discover microRNAs whose expression changes following radiation in breast and prostate cancer: We set out to screen for microRNAs whose expression is regulated following treatment with ionizing radiation, reasoning that the regulated microRNAs may contribute to the DNA damage response of cells and may be involved in mechanisms of radiation resistance of cancers.

Goal 2: Discover microRNAs differentially expressed between radiation-sensitive and -resistant cancer cell lines. Determining which microRNAs correlate with radiation sensitivity should provide new insight into genetic mechanisms by which cancers become resistant to ionizing 
radiation and cytotoxic chemotherapy. Combining the knowledge of which microRNAs change in expression upon radiation with an understanding of which microRNAs confer radiation sensitivity may provide new tools to predict radiation responsiveness and to sensitize cancers to treatment with fractionated radiotherapy and improve survival.

\section{ii. The role of long noncoding RNAs in skeletal myogenesis:}

Goal 1: Discover long noncoding RNAs whose expression are upregulated during myogenesis using $\mathrm{C} 2 \mathrm{C} 12$ myoblasts as they differentiate into myotubes in culture. This should both provide examples of long noncoding RNAs involved in skeletal myogenesis, as well as lncRNAs that are upregulated during terminal differentiation in general.

Goal 2: Determine the effect of modulating expression of novel long noncoding RNAs in skeletal myogenesis, and determine their mechanism of action. Discovering novel lncRNAs whose depletion has a phenotypic effect in skeletal myogenesis will yield insight into how lncRNAs regulated muscle development and development in general. Discovering lncRNAs that are important for normal development should also provide targets for study in the context of diseases that are marked by defects in development, stem cell renewal and regeneration of adult tissue. 


\section{F. References:}

1. Crick F. Central dogma of molecular biology. Nature. 1970 Aug 8;227(5258):561-3.

2. Venter JC, Adams MD, Myers EW, Li PW, Mural RJ, Sutton GG, et al. The sequence of the human genome. Science. 2001 Feb 16;291(5507):1304-51.

3. Consortium EP, Birney E, Stamatoyannopoulos JA, Dutta A, Guigo R, Gingeras TR, et al. Identification and analysis of functional elements in $1 \%$ of the human genome by the ENCODE pilot project. Nature. 2007 Jun 14;447(7146):799-816.

4. Wang KC, Chang HY. Molecular Mechanisms of Long Noncoding RNAs. Molecular Cell. 2011;43(6):904-14.

5. Wapinski O, Chang HY. Long noncoding RNAs and human disease. Trends Cell Biol. 2011 Jun;21(6):354-61.

6. Nissen P, Hansen J, Ban N, Moore PB, Steitz TA. The structural basis of ribosome activity in peptide bond synthesis. Science. 2000 Aug 11;289(5481):920-30.

7. He L, Hannon GJ. MicroRNAs: small RNAs with a big role in gene regulation. Nat Rev Genet. 2004 Jul;5(7):522-31.

8. Guttman M, Garber M, Levin JZ, Donaghey J, Robinson J, Adiconis X, et al. Ab initio reconstruction of cell type-specific transcriptomes in mouse reveals the conserved multi-exonic structure of lincRNAs. Nat Biotechnol. 2010 May;28(5):503-10.

9. Guttman M, Amit I, Garber M, French C, Lin MF, Feldser D, et al. Chromatin signature reveals over a thousand highly conserved large non-coding RNAs in mammals. Nature. 2009 Mar 12;458(7235):223-7.

10. Cabili MN, Trapnell C, Goff L, Koziol M, Tazon-Vega B, Regev A, et al. Integrative annotation of human large intergenic noncoding RNAs reveals global properties and specific subclasses. Genes Dev. 2011 Sep 15;25(18):1915-27.

11. Reinhart BJ, Slack FJ, Basson M, Pasquinelli AE, Bettinger JC, Rougvie AE, et al. The 21-nucleotide let-7 RNA regulates developmental timing in Caenorhabditis elegans. Nature. 2000 Feb 24;403(6772):901-6.

12. Calin GA, Croce CM. MicroRNA signatures in human cancers. Nat Rev Cancer. 2006 Nov;6(11):857-66.

13. Chen K, Rajewsky N. The evolution of gene regulation by transcription factors and microRNAs. Nat Rev Genet. 2007 Feb;8(2):93-103.

14. Durocher D, Jackson SP. DNA-PK, ATM and ATR as sensors of DNA damage: variations on a theme? Curr Opin Cell Biol. 2001 Apr;13(2):225-31.

15. Jazayeri A, Falck J, Lukas C, Bartek J, Smith GC, Lukas J, et al. ATM- and cell cycledependent regulation of ATR in response to DNA double-strand breaks. Nat Cell Biol. 2006 Jan;8(1):37-45.

16. Roy R, Chun J, Powell SN. BRCA1 and BRCA2: different roles in a common pathway of genome protection. Nat Rev Cancer. 2012 Jan;12(1):68-78.

17. Wang Y, Taniguchi T. MicroRNAs and DNA damage response: Implications for cancer therapy. Cell Cycle. 2013;12(1):32-42.

18. Caestecker KW, Van de Walle GR. The role of BRCA1 in DNA double-strand repair: past and present. Exp Cell Res. 2013 Mar 10;319(5):575-87. 
19. Nakamura K, Kato A, Kobayashi J, Yanagihara H, Sakamoto S, Oliveira DV, et al. Regulation of homologous recombination by RNF20-dependent H2B ubiquitination. Mol Cell. 2011 Mar 4;41(5):515-28.

20. Wang C, Lees-Miller SP. Detection and Repair of Ionizing Radiation-Induced DNA Double Strand Breaks: New Developments in Nonhomologous End Joining. Int J Radiat Oncol Biol Phys. 2013 Feb 20.

21. Lan L, Ui A, Nakajima S, Hatakeyama K, Hoshi M, Watanabe R, et al. The ACF1 complex is required for DNA double-strand break repair in human cells. Mol Cell. 2010 Dec 22;40(6):976-87.

22. Sun D, Lee YS, Malhotra A, Kim HK, Matecic M, Evans C, et al. miR-99 family of MicroRNAs suppresses the expression of prostate-specific antigen and prostate cancer cell proliferation. Cancer Res. 2011 Feb 15;71(4):1313-24.

23. Yoo AS, Staahl BT, Chen L, Crabtree GR. MicroRNA-mediated switching of chromatinremodelling complexes in neural development. Nature. 2009 Jul 30;460(7255):642-6.

24. Sakurai K, Furukawa C, Haraguchi T, Inada K, Shiogama K, Tagawa T, et al. MicroRNAs miR-199a-5p and -3p target the Brm subunit of SWI/SNF to generate a doublenegative feedback loop in a variety of human cancers. Cancer Res. 2011 Mar 1;71(5):1680-9. 25. Lee YS, Dutta A. MicroRNAs in cancer. Annu Rev Pathol. 2009;4:199-227.

26. Suzuki HI, Yamagata K, Sugimoto K, Iwamoto T, Kato S, Miyazono K. Modulation of microRNA processing by p53. Nature. [10.1038/nature08199]. 2009;460(7254):529-33.

27. Yamakuchi M, Lowenstein CJ. MiR-34, SIRT1 and p53: the feedback loop. Cell Cycle. 2009 Mar 1;8(5):712-5.

28. He L, He X, Lim LP, de Stanchina E, Xuan Z, Liang Y, et al. A microRNA component of the p53 tumour suppressor network. Nature. 2007 Jun 28;447(7148):1130-4.

29. Simone NL, Soule BP, Ly D, Saleh AD, Savage JE, Degraff W, et al. Ionizing radiationinduced oxidative stress alters miRNA expression. PLoS One. 2009;4(7):e6377.

30. Wagner-Ecker M, Schwager C, Wirkner U, Abdollahi A, Huber PE. MicroRNA expression after ionizing radiation in human endothelial cells. Radiat Oncol. 2010;5:25.

31. Mueller AC, Sun D, Dutta A. The miR-99 family regulates the DNA damage response through its target SNF2H. Oncogene. 2013 Feb 28;32(9):1164-72.

32. Chan JA, Krichevsky AM, Kosik KS. MicroRNA-21 is an antiapoptotic factor in human glioblastoma cells. Cancer Res. 2005 Jul 15;65(14):6029-33.

33. Si ML, Zhu S, Wu H, Lu Z, Wu F, Mo YY. miR-21-mediated tumor growth. Oncogene. 2007 Apr 26;26(19):2799-803.

34. Fulci V, Chiaretti S, Goldoni M, Azzalin G, Carucci N, Tavolaro S, et al. Quantitative technologies establish a novel microRNA profile of chronic lymphocytic leukemia. Blood. 2007 Jun 1;109(11):4944-51.

35. Iorio MV, Ferracin M, Liu CG, Veronese A, Spizzo R, Sabbioni S, et al. MicroRNA gene expression deregulation in human breast cancer. Cancer Res. 2005 Aug 15;65(16):7065-70.

36. Volinia S, Calin GA, Liu CG, Ambs S, Cimmino A, Petrocca F, et al. A microRNA expression signature of human solid tumors defines cancer gene targets. Proc Natl Acad Sci U S A. 2006 Feb 14;103(7):2257-61.

37. Chaudhry MA, Sachdeva H, Omaruddin RA. Radiation-induced micro-RNA modulation in glioblastoma cells differing in DNA-repair pathways. DNA Cell Biol. 2010 Sep;29(9):553-61. 
38. Weidhaas JB, Babar I, Nallur SM, Trang P, Roush S, Boehm M, et al. MicroRNAs as potential agents to alter resistance to cytotoxic anticancer therapy. Cancer Res. 2007 Dec 1;67(23):11111-6.

39. Johnson SM, Grosshans H, Shingara J, Byrom M, Jarvis R, Cheng A, et al. RAS is regulated by the let-7 microRNA family. Cell. 2005 Mar 11;120(5):635-47.

40. Takamizawa J, Konishi H, Yanagisawa K, Tomida S, Osada H, Endoh H, et al. Reduced expression of the let-7 microRNAs in human lung cancers in association with shortened postoperative survival. Cancer Res. 2004 Jun 1;64(11):3753-6.

41. Dickey JS, Zemp FJ, Martin OA, Kovalchuk O. The role of miRNA in the direct and indirect effects of ionizing radiation. Radiat Environ Biophys. 2011 Nov;50(4):491-9.

42. Cha HJ, Seong KM, Bae S, Jung JH, Kim CS, Yang KH, et al. Identification of specific microRNAs responding to low and high dose gamma-irradiation in the human lymphoblast line IM9. Oncol Rep. 2009 Oct;22(4):863-8.

43. Chaudhry MA, Omaruddin RA, Kreger B, de Toledo SM, Azzam EI. Micro RNA responses to chronic or acute exposures to low dose ionizing radiation. Mol Biol Rep. 2012 Jul;39(7):7549-58.

44. Girardi C, De Pitta C, Casara S, Sales G, Lanfranchi G, Celotti L, et al. Analysis of miRNA and mRNA expression profiles highlights alterations in ionizing radiation response of human lymphocytes under modeled microgravity. PLoS One. 2012;7(2):e31293.

45. Josson S, Sung SY, Lao K, Chung LW, Johnstone PA. Radiation modulation of microRNA in prostate cancer cell lines. Prostate. 2008 Nov 1;68(15):1599-606.

46. Krishnan K, Steptoe AL, Martin HC, Wani S, Nones K, Waddell N, et al. MicroRNA182-5p targets a network of genes involved in DNA repair. RNA. 2013 Feb;19(2):230-42.

47. Moskwa P, Buffa FM, Pan Y, Panchakshari R, Gottipati P, Muschel RJ, et al. miR-182mediated downregulation of BRCA1 impacts DNA repair and sensitivity to PARP inhibitors. Mol Cell. 2011 Jan 21;41(2):210-20.

48. $\quad$ Song $\mathrm{L}$, Lin $\mathrm{C}, \mathrm{Wu} \mathrm{Z}$, Gong $\mathrm{H}$, Zeng $\mathrm{Y}, \mathrm{Wu}$ J, et al. miR-18a impairs DNA damage response through downregulation of ataxia telangiectasia mutated (ATM) kinase. PLoS One. 2011;6(9):e25454.

49. Ng WL, Yan D, Zhang X, Mo YY, Wang Y. Over-expression of miR-100 is responsible for the low-expression of ATM in the human glioma cell line: M059J. DNA Repair (Amst). 2010 Nov 10;9(11):1170-5.

50. Yan D, Ng WL, Zhang X, Wang P, Zhang Z, Mo YY, et al. Targeting DNA-PKcs and ATM with miR-101 sensitizes tumors to radiation. PLoS One. 2010;5(7):e11397.

51. Hu H, Du L, Nagabayashi G, Seeger RC, Gatti RA. ATM is down-regulated by N-Mycregulated microRNA-421. Proc Natl Acad Sci U S A. 2010 Jan 26;107(4):1506-11.

52. Wang Y, Yu Y, Tsuyada A, Ren X, Wu X, Stubblefield K, et al. Transforming growth factor-beta regulates the sphere-initiating stem cell-like feature in breast cancer through miRNA181 and ATM. Oncogene. 2011 Mar 24;30(12):1470-80.

53. Lukas C, Bartkova J, Latella L, Falck J, Mailand N, Schroeder T, et al. DNA damageactivated kinase Chk2 is independent of proliferation or differentiation yet correlates with tissue biology. Cancer Res. 2001 Jul 1;61(13):4990-3.

54. Yaneva M, Jhiang S. Expression of the Ku protein during cell proliferation. Biochim Biophys Acta. 1991 Oct 8;1090(2):181-7. 
55. Lal A, Pan Y, Navarro F, Dykxhoorn DM, Moreau L, Meire E, et al. miR-24-mediated downregulation of H2AX suppresses DNA repair in terminally differentiated blood cells. Nat Struct Mol Biol. 2009 May;16(5):492-8.

56. Lee SO, Masyuk T, Splinter P, Banales JM, Masyuk A, Stroope A, et al. MicroRNA15a modulates expression of the cell-cycle regulator $\mathrm{Cdc} 25 \mathrm{~A}$ and affects hepatic cystogenesis in a rat model of polycystic kidney disease. J Clin Invest. 2008 Nov;118(11):3714-24.

57. Pothof J, Verkaik NS, van IW, Wiemer EA, Ta VT, van der Horst GT, et al. MicroRNAmediated gene silencing modulates the UV-induced DNA-damage response. EMBO J. $2009 \mathrm{Jul}$ 22;28(14):2090-9.

58. de Oliveira PE, Zhang L, Wang Z, Lazo JS. Hypoxia-mediated regulation of Cdc25A phosphatase by p21 and miR-21. Cell Cycle. 2009 Oct 1;8(19):3157-64.

59. Bartek J, Lukas J. Chk1 and Chk2 kinases in checkpoint control and cancer. Cancer Cell. 2003 May;3(5):421-9.

60. Le MT, Teh C, Shyh-Chang N, Xie H, Zhou B, Korzh V, et al. MicroRNA-125b is a novel negative regulator of p53. Genes Dev. 2009 Apr 1;23(7):862-76.

61. Le MT, Shyh-Chang N, Khaw SL, Chin L, Teh C, Tay J, et al. Conserved regulation of p53 network dosage by microRNA-125b occurs through evolving miRNA-target gene pairs. PLoS Genet. 2011 Sep;7(9):e1002242.

62. Nishida N, Yokobori T, Mimori K, Sudo T, Tanaka F, Shibata K, et al. MicroRNA miR$125 \mathrm{~b}$ is a prognostic marker in human colorectal cancer. Int J Oncol. 2011 May;38(5):1437-43.

63. Enomoto Y, Kitaura J, Hatakeyama K, Watanuki J, Akasaka T, Kato N, et al. Emu/miR$125 \mathrm{~b}$ transgenic mice develop lethal B-cell malignancies. Leukemia. 2011 Dec;25(12):1849-56. 64. Zhang Y, Yan LX, Wu QN, Du ZM, Chen J, Liao DZ, et al. miR-125b is methylated and functions as a tumor suppressor by regulating the ETS1 proto-oncogene in human invasive breast cancer. Cancer Res. 2011 May 15;71(10):3552-62.

65. Hu W, Chan CS, Wu R, Zhang C, Sun Y, Song JS, et al. Negative regulation of tumor suppressor p53 by microRNA miR-504. Mol Cell. 2010 Jun 11;38(5):689-99.

66. Hermeking H. MicroRNAs in the p53 network: micromanagement of tumour suppression. Nat Rev Cancer. 2012 Sep;12(9):613-26.

67. Brown CJ, Hendrich BD, Rupert JL, Lafreniere RG, Xing Y, Lawrence J, et al. The human XIST gene: analysis of a $17 \mathrm{~kb}$ inactive X-specific RNA that contains conserved repeats and is highly localized within the nucleus. Cell. 1992 Oct 30;71(3):527-42.

68. Plath K, Talbot D, Hamer KM, Otte AP, Yang TP, Jaenisch R, et al. Developmentally regulated alterations in Polycomb repressive complex 1 proteins on the inactive $\mathrm{X}$ chromosome. J Cell Biol. 2004 Dec 20;167(6):1025-35.

69. Marks H, Chow JC, Denissov S, Francoijs KJ, Brockdorff N, Heard E, et al. Highresolution analysis of epigenetic changes associated with X inactivation. Genome Res. 2009 Aug;19(8):1361-73.

70. Lee JT, Davidow LS, Warshawsky D. Tsix, a gene antisense to Xist at the X-inactivation centre. Nat Genet. 1999 Apr;21(4):400-4.

71. Pontier DB, Gribnau J. Xist regulation and function explored. Hum Genet. 2011 Aug;130(2):223-36.

72. Gupta RA, Shah N, Wang KC, Kim J, Horlings HM, Wong DJ, et al. Long non-coding RNA HOTAIR reprograms chromatin state to promote cancer metastasis. Nature. 2010 Apr 15;464(7291):1071-6. 
73. Bhan A, Hussain I, Ansari KI, Kasiri S, Bashyal A, Mandal SS. Antisense Transcript Long Noncoding RNA (IncRNA) HOTAIR is Transcriptionally Induced by Estradiol. J Mol Biol. 2013 Jan 31.

74. Yang Z, Zhou L, Wu LM, Lai MC, Xie HY, Zhang F, et al. Overexpression of long noncoding RNA HOTAIR predicts tumor recurrence in hepatocellular carcinoma patients following liver transplantation. Ann Surg Oncol. 2011 May;18(5):1243-50.

75. Ishibashi M, Kogo R, Shibata K, Sawada G, Takahashi Y, Kurashige J, et al. Clinical significance of the expression of long non-coding RNA HOTAIR in primary hepatocellular carcinoma. Oncol Rep. 2013 Mar;29(3):946-50.

76. Nie Y, Liu X, Qu S, Song E, Zou H, Gong C. Long non-coding RNA HOTAIR is an independent prognostic marker for nasopharyngeal carcinoma progression and survival. Cancer Sci. 2013 Apr;104(4):458-64.

77. Schorderet P, Duboule D. Structural and functional differences in the long non-coding RNA hotair in mouse and human. PLoS Genet. 2011 May;7(5):e1002071.

78. Orom UA, Derrien T, Beringer M, Gumireddy K, Gardini A, Bussotti G, et al. Long noncoding RNAs with enhancer-like function in human cells. Cell. 2010 Oct 1;143(1):46-58.

79. Orom UA, Derrien T, Guigo R, Shiekhattar R. Long noncoding RNAs as enhancers of gene expression. Cold Spring Harb Symp Quant Biol. 2010;75:325-31.

80. Kim TK, Hemberg M, Gray JM, Costa AM, Bear DM, Wu J, et al. Widespread transcription at neuronal activity-regulated enhancers. Nature. 2010 May 13;465(7295):182-7.

81. De Santa F, Barozzi I, Mietton F, Ghisletti S, Polletti S, Tusi BK, et al. A large fraction of extragenic RNA pol II transcription sites overlap enhancers. PLoS Biol. 2010 May;8(5):e1000384.

82. Onoguchi M, Hirabayashi Y, Koseki H, Gotoh Y. A noncoding RNA regulates the neurogenin 1 gene locus during mouse neocortical development. Proc Natl Acad Sci U S A. 2012 Oct 16;109(42):16939-44.

83. Maass PG, Rump A, Schulz H, Stricker S, Schulze L, Platzer K, et al. A misplaced lncRNA causes brachydactyly in humans. J Clin Invest. 2012 Nov 1;122(11):3990-4002.

84. Lai F, Orom UA, Cesaroni M, Beringer M, Taatjes DJ, Blobel GA, et al. Activating RNAs associate with Mediator to enhance chromatin architecture and transcription. Nature. 2013 Feb 17.

85. Gomez JA, Wapinski OL, Yang YW, Bureau JF, Gopinath S, Monack DM, et al. The NeST long ncRNA controls microbial susceptibility and epigenetic activation of the interferongamma locus. Cell. 2013 Feb 14;152(4):743-54.

86. Lanz RB, McKenna NJ, Onate SA, Albrecht U, Wong J, Tsai SY, et al. A steroid receptor coactivator, SRA, functions as an RNA and is present in an SRC-1 complex. Cell. 1999 Apr 2;97(1):17-27.

87. Bates GJ, Nicol SM, Wilson BJ, Jacobs AM, Bourdon JC, Wardrop J, et al. The DEAD box protein p68: a novel transcriptional coactivator of the p53 tumour suppressor. EMBO J. 2005 Feb 9;24(3):543-53.

88. Caretti G, Schiltz RL, Dilworth FJ, Di Padova M, Zhao P, Ogryzko V, et al. The RNA helicases p68/p72 and the noncoding RNA SRA are coregulators of MyoD and skeletal muscle differentiation. Dev Cell. 2006 Oct;11(4):547-60.

89. Hube F, Velasco G, Rollin J, Furling D, Francastel C. Steroid receptor RNA activator protein binds to and counteracts SRA RNA-mediated activation of MyoD and muscle differentiation. Nucleic Acids Res. 2011 Jan;39(2):513-25. 
90. Luo JH, Ren B, Keryanov S, Tseng GC, Rao UN, Monga SP, et al. Transcriptomic and genomic analysis of human hepatocellular carcinomas and hepatoblastomas. Hepatology. 2006 Oct;44(4):1012-24.

91. Guffanti A, Iacono M, Pelucchi P, Kim N, Solda G, Croft LJ, et al. A transcriptional sketch of a primary human breast cancer by 454 deep sequencing. BMC Genomics. 2009;10:163. 92. Gutschner T, Hammerle M, Eissmann M, Hsu J, Kim Y, Hung G, et al. The noncoding RNA MALAT1 is a critical regulator of the metastasis phenotype of lung cancer cells. Cancer Res. 2013 Feb 1;73(3):1180-9.

93. Rajaram V, Knezevich S, Bove KE, Perry A, Pfeifer JD. DNA sequence of the translocation breakpoints in undifferentiated embryonal sarcoma arising in mesenchymal hamartoma of the liver harboring the $\mathrm{t}(11 ; 19)(\mathrm{q} 11 ; \mathrm{q} 13.4)$ translocation. Genes Chromosomes Cancer. 2007 May;46(5):508-13.

94. Argani P, Yonescu R, Morsberger L, Morris K, Netto GJ, Smith N, et al. Molecular confirmation of $\mathrm{t}(6 ; 11)(\mathrm{p} 21 ; \mathrm{q} 12)$ renal cell carcinoma in archival paraffin-embedded material using a break-apart TFEB FISH assay expands its clinicopathologic spectrum. Am J Surg Pathol. 2012 Oct;36(10):1516-26.

95. Tripathi V, Shen Z, Chakraborty A, Giri S, Freier SM, Wu X, et al. Long Noncoding RNA MALAT1 Controls Cell Cycle Progression by Regulating the Expression of Oncogenic Transcription Factor B-MYB. PLoS Genet. 2013 Mar;9(3):e1003368.

96. Bernard D, Prasanth KV, Tripathi V, Colasse S, Nakamura T, Xuan Z, et al. A long nuclear-retained non-coding RNA regulates synaptogenesis by modulating gene expression. EMBO J. 2010 Sep 15;29(18):3082-93.

97. Brown JA, Valenstein ML, Yario TA, Tycowski KT, Steitz JA. Formation of triplehelical structures by the 3'-end sequences of MALAT1 and MENbeta noncoding RNAs. Proc Natl Acad Sci U S A. 2012 Nov 20;109(47):19202-7.

98. Wilusz JE, JnBaptiste CK, Lu LY, Kuhn CD, Joshua-Tor L, Sharp PA. A triple helix stabilizes the 3' ends of long noncoding RNAs that lack poly(A) tails. Genes Dev. 2012 Nov 1;26(21):2392-407.

99. Zhang B, Arun G, Mao YS, Lazar Z, Hung G, Bhattacharjee G, et al. The lncRNA Malat 1 is dispensable for mouse development but its transcription plays a cis-regulatory role in the adult. Cell Rep. 2012 Jul 26;2(1):111-23.

100. Eissmann M, Gutschner T, Hammerle M, Gunther S, Caudron-Herger M, Gross M, et al. Loss of the abundant nuclear non-coding RNA MALAT1 is compatible with life and development. RNA Biol. 2012 Aug;9(8):1076-87.

101. Watts R, Johnsen VL, Shearer J, Hittel DS. The myostatin-induced inhibition of the long noncoding RNA Malat1 is associated with decreased myogenesis. Am J Physiol Cell Physiol. 2013 Mar 13.

102. Ebert MS, Neilson JR, Sharp PA. MicroRNA sponges: competitive inhibitors of small RNAs in mammalian cells. Nat Methods. 2007 Sep;4(9):721-6.

103. Salmena L, Poliseno L, Tay Y, Kats L, Pandolfi PP. A ceRNA hypothesis: the Rosetta Stone of a hidden RNA language? Cell. 2011 Aug 5;146(3):353-8.

104. Cesana M, Cacchiarelli D, Legnini I, Santini T, Sthandier O, Chinappi M, et al. A long noncoding RNA controls muscle differentiation by functioning as a competing endogenous RNA. Cell. 2011 Oct 14;147(2):358-69. 
105. Wang Y, Xu Z, Jiang J, Xu C, Kang J, Xiao L, et al. Endogenous miRNA Sponge lincRNA-RoR Regulates Oct4, Nanog, and Sox2 in Human Embryonic Stem Cell Self-Renewal. Dev Cell. 2013 Apr 15;25(1):69-80.

106. Haraguchi T, Ozaki Y, Iba H. Vectors expressing efficient RNA decoys achieve the longterm suppression of specific microRNA activity in mammalian cells. Nucleic Acids Res. 2009 Apr;37(6):e43.

107. Hansen TB, Wiklund ED, Bramsen JB, Villadsen SB, Statham AL, Clark SJ, et al. miRNA-dependent gene silencing involving Ago2-mediated cleavage of a circular antisense RNA. EMBO J. 2011 Nov 2;30(21):4414-22.

108. Hansen TB, Jensen TI, Clausen BH, Bramsen JB, Finsen B, Damgaard CK, et al. Natural RNA circles function as efficient microRNA sponges. Nature. 2013 Mar 21;495(7441):384-8.

109. Memczak S, Jens M, Elefsinioti A, Torti F, Krueger J, Rybak A, et al. Circular RNAs are a large class of animal RNAs with regulatory potency. Nature. 2013 Mar 21;495(7441):333-8. 110. Yokoyama S, Asahara H. The myogenic transcriptional network. Cell Mol Life Sci. 2011 Jun;68(11):1843-9.

111. Lassar AB, Paterson BM, Weintraub H. Transfection of a DNA locus that mediates the conversion of 10T1/2 fibroblasts to myoblasts. Cell. 1986 Dec 5;47(5):649-56.

112. Choi J, Costa ML, Mermelstein CS, Chagas C, Holtzer S, Holtzer H. MyoD converts primary dermal fibroblasts, chondroblasts, smooth muscle, and retinal pigmented epithelial cells into striated mononucleated myoblasts and multinucleated myotubes. Proc Natl Acad Sci U S A. 1990 Oct;87(20):7988-92.

113. Berkes CA, Tapscott SJ. MyoD and the transcriptional control of myogenesis. Semin Cell Dev Biol. 2005 Aug-Oct;16(4-5):585-95.

114. Braun T, Buschhausen-Denker G, Bober E, Tannich E, Arnold HH. A novel human muscle factor related to but distinct from MyoD1 induces myogenic conversion in 10T1/2 fibroblasts. EMBO J. 1989 Mar;8(3):701-9.

115. Nabeshima Y, Hanaoka K, Hayasaka M, Esumi E, Li S, Nonaka I. Myogenin gene disruption results in perinatal lethality because of severe muscle defect. Nature. 1993 Aug 5;364(6437):532-5.

116. Lassar AB, Buskin JN, Lockshon D, Davis RL, Apone S, Hauschka SD, et al. MyoD is a sequence-specific DNA binding protein requiring a region of myc homology to bind to the muscle creatine kinase enhancer. Cell. 1989 Sep 8;58(5):823-31.

117. Rudnicki MA, Braun T, Hinuma S, Jaenisch R. Inactivation of MyoD in mice leads to up-regulation of the myogenic HLH gene Myf-5 and results in apparently normal muscle development. Cell. 1992 Oct 30;71(3):383-90.

118. Rudnicki MA, Schnegelsberg PN, Stead RH, Braun T, Arnold HH, Jaenisch R. MyoD or Myf-5 is required for the formation of skeletal muscle. Cell. 1993 Dec 31;75(7):1351-9.

119. Megeney LA, Kablar B, Garrett K, Anderson JE, Rudnicki MA. MyoD is required for myogenic stem cell function in adult skeletal muscle. Genes Dev. 1996 May 15;10(10):1173-83.

120. Wang Y, Jaenisch R. Myogenin can substitute for Myf5 in promoting myogenesis but less efficiently. Development. 1997 Jul;124(13):2507-13.

121. Kassar-Duchossoy L, Gayraud-Morel B, Gomes D, Rocancourt D, Buckingham M, Shinin V, et al. Mrf4 determines skeletal muscle identity in Myf5:Myod double-mutant mice. Nature. 2004 Sep 23;431(7007):466-71.

122. Sumariwalla VM, Klein WH. Similar myogenic functions for myogenin and MRF4 but not MyoD in differentiated murine embryonic stem cells. Genesis. 2001 Aug;30(4):239-49. 
123. Blackwell TK, Weintraub H. Differences and similarities in DNA-binding preferences of MyoD and E2A protein complexes revealed by binding site selection. Science. $1990 \mathrm{Nov}$ 23;250(4984):1104-10.

124. Kumar D, Shadrach JL, Wagers AJ, Lassar AB. Id3 is a direct transcriptional target of Pax7 in quiescent satellite cells. Mol Biol Cell. 2009 Jul;20(14):3170-7.

125. Benezra R, Davis RL, Lockshon D, Turner DL, Weintraub H. The protein Id: a negative regulator of helix-loop-helix DNA binding proteins. Cell. 1990 Apr 6;61(1):49-59.

126. Lin Q, Schwarz J, Bucana C, Olson EN. Control of mouse cardiac morphogenesis and myogenesis by transcription factor MEF2C. Science. 1997 May 30;276(5317):1404-7.

127. Molkentin JD, Black BL, Martin JF, Olson EN. Cooperative activation of muscle gene expression by MEF2 and myogenic bHLH proteins. Cell. 1995 Dec 29;83(7):1125-36.

128. Gagan J, Dey BK, Layer R, Yan Z, Dutta A. Notch3 and Mef2c proteins are mutually antagonistic via Mkp1 protein and miR-1/206 microRNAs in differentiating myoblasts. J Biol Chem. 2012 Nov 23;287(48):40360-70.

129. Puri PL, Sartorelli V, Yang XJ, Hamamori Y, Ogryzko VV, Howard BH, et al. Differential roles of p300 and PCAF acetyltransferases in muscle differentiation. Mol Cell. 1997 Dec;1(1):35-45.

130. Mal A, Sturniolo M, Schiltz RL, Ghosh MK, Harter ML. A role for histone deacetylase HDAC1 in modulating the transcriptional activity of MyoD: inhibition of the myogenic program. EMBO J. 2001 Apr 2;20(7):1739-53.

131. Li ZY, Yang J, Gao X, Lu JY, Zhang Y, Wang K, et al. Sequential recruitment of PCAF and BRG1 contributes to myogenin activation in 12-O-tetradecanoylphorbol-13-acetate-induced early differentiation of rhabdomyosarcoma-derived cells. J Biol Chem. 2007 Jun 29;282(26):18872-8.

132. Gagan J, Dey BK, Dutta A. MicroRNAs regulate and provide robustness to the myogenic transcriptional network. Curr Opin Pharmacol. 2012 Jun;12(3):383-8.

133. Blum R, Vethantham V, Bowman C, Rudnicki M, Dynlacht BD. Genome-wide identification of enhancers in skeletal muscle: the role of MyoD1. Genes Dev. 2012 Dec 15;26(24):2763-79.

134. Borensztein M, Monnier P, Court F, Louault Y, Ripoche MA, Tiret L, et al. Myod and H19-Igf2 locus interactions are required for diaphragm formation in the mouse. Development. 2013 Mar;140(6):1231-9.

135. Pandorf CE, Jiang W, Qin AX, Bodell PW, Baldwin KM, Haddad F. Regulation of an antisense RNA with the transition of neonatal to IIb myosin heavy chain during postnatal development and hypothyroidism in rat skeletal muscle. Am J Physiol Regul Integr Comp Physiol. 2012 Apr;302(7):R854-67. 


\section{G. Figures.}
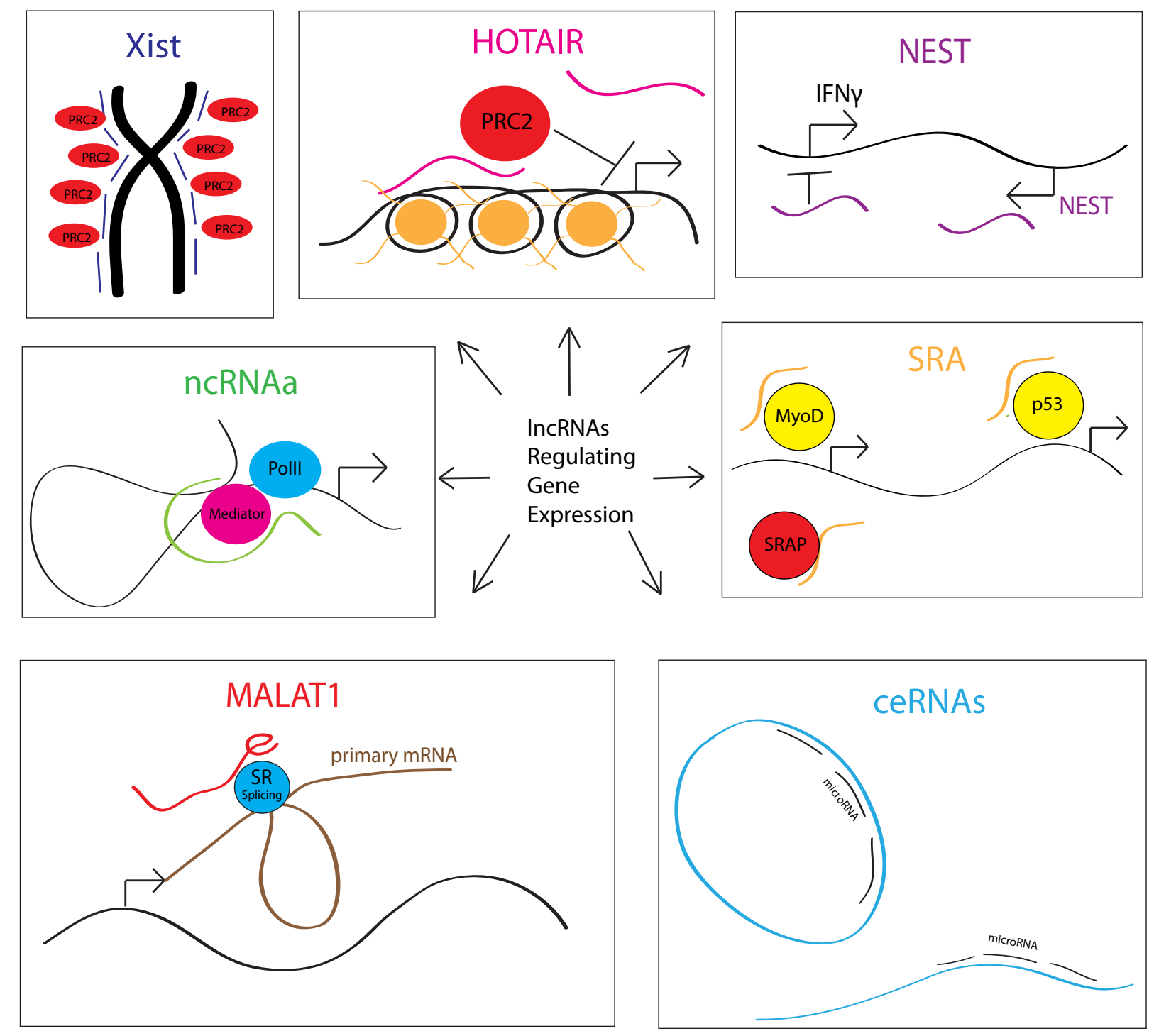

Figure 1. Models of IncRNA regulation of gene expression. 


\section{Chapter 2 \\ Adapted from: The miR-99 family regulates the DNA damage response through its target SNF2H.}

Mueller AC, Sun D, Dutta A.

Oncogene. 2013 Feb 28;32(9):1164-72. doi: 10.1038/onc.2012.131. Epub 2012 Apr 23.

\section{Abstract:}

Chromatin remodeling factors are becoming known as crucial facilitators of recruitment of repair proteins to sites of DNA damage. Multiple chromatin remodeling protein complexes are now known to be required for efficient double strand break repair. In a screen for microRNAs that modulate the DNA damage response, we discovered that expression of the miR-99 family of microRNAs correlates with radiation sensitivity. These microRNAs were also transiently induced following radiation. The microRNAs target the SWI/SNF chromatin remodeling factor SNF2H/SMARCA5, a component of the ACF1 complex. We found that by reducing levels of SNF2H, miR-99a reduced BRCA1 localization to sites of DNA damage. Introduction of the miR-99 family of microRNAs into cells reduced the rate and overall efficiency of repair by both homologous recombination and non-homologous end joining. Finally, induction of the miR-99 family following radiation prevents an increase in SNF2H expression and reduces the recruitment of BRCA1 to sites of DNA damage following a second dose of radiation, reducing the efficiency of repair after multiple rounds of radiation as used in fractionated radiotherapy.

Contribution: All experiments were designed and performed by Adam Mueller. Dandan Sun contributed to the design of the study. All of the work was supervised by Anindya Dutta. 


\title{
The miR-99 family regulates the DNA damage response through its target SNF2H.
}

\author{
Adam C. Mueller ${ }^{1}$, Dandan Sun ${ }^{1}$, Anindya Dutta ${ }^{1}$. \\ ${ }^{1}$ Department of Biochemistry and Molecular Genetics, University of Virginia School of \\ Medicine, Jordan Hall 1232, Charlottesville, Virginia 22908 \\ *Corresponding Author
}

\section{Introduction}

Mammalian cells have developed multiple complex mechanisms for identifying and repairing damage to their genomes caused by a wide variety of sources. Damage caused by UV, ionizing radiation, cross-linking and intercalating agents, as well as DNA replication errors are identified and repaired through multiple pathways dependent on the type of damage incurred. The ability of normal cells to detect and repair DNA damage, as well as initiate apoptosis when excess damage has occurred is crucial for maintaining genomic stability and preventing cancer. Conversely, the ability of cancer cells to sustain and repair large quantities of DNA damage contributes to their frequently observed resistance to DNA damaging chemotherapeutic and radiation treatment. Several histone modifications specifically designate sites of DNA damage, and are required for the recruitment of repair proteins(1). In particular, phosphorylation of H2AX $(\gamma \mathrm{H} 2 \mathrm{AX})$ is required for DNA damage signaling and repair (2). There is a steadily growing body of evidence that indicates several other histone modifications including ubiquitination, methylation and acetylation occur that play a role in conjunction with, as well as independent of, $\gamma \mathrm{H} 2 \mathrm{AX}$ signaling to facilitate effective DNA repair $(1,3)$.

One of the functions of these histone marks is to recruit chromatin remodeling complexes to give repair proteins access to sites of DNA damage. ATP-dependent chromatin remodeling 
complexes were initially implicated in DSB repair when it was discovered that the INO80 complex is recruited to phosphorylated $\mathrm{H} 2 \mathrm{~A}$ in budding yeast and required for efficient conversion of double strand breaks into single stranded DNA prior to repair (4-7). The SWI/SNF complex is also recruited to $\gamma \mathrm{H} 2 \mathrm{AX}$ in conjunction with acetylation of histone $\mathrm{H} 3$, and required for maximal phosphorylation of $\mathrm{H} 2 \mathrm{AX}$ (8). Conversely, the Tip60 complex is recruited to DNA damage sites to dephosphorylate $\gamma \mathrm{H} 2 \mathrm{AX}$ after the damage is repaired (9).

Double strand break(DSB) repair in mammalian cells typically occurs through two distinct pathways, homologous recombination(HR) or non-homologous end joining(NHEJ). DSB repair through HR is dependent on the recruitment of BRCA1 to DSB sites through a complex signaling cascade downstream of $\gamma \mathrm{H} 2 \mathrm{AX}(1)$. The protein MDC1 binds to $\gamma \mathrm{H} 2 \mathrm{AX}(10)$ and recruits the ubiquitin ligase $\mathrm{RNF}$, which subsequently ubiquitinates $\mathrm{H} 2 \mathrm{~A}$ and $\mathrm{H} 2 \mathrm{AX}$ leading to eventual BRCA1 recruitment (11-14). NHEJ involves the binding of Ku proteins to double stranded ends of DNA at break sites(15) and the recruitment of DNA-PKcs and DNA ligase IV, which then facilitate the ligation of the free ends (16). Both processes require the action of chromatin remodeling proteins for proper recruitment of repair proteins to double strand break $\operatorname{sites}(17,18)$.

Recently, the chromatin remodeling complex SNF2H (also known as SMARCA5) has been implicated in both the HR and NHEJ DNA repair pathways. Following DNA damage, the ACF1 chromatin remodeling complex containing SNF2H is required for the efficient recruitment of $\mathrm{Ku} 70 / 80$ proteins to laser stripe induced DNA damage sites, and depletion of components of this complex result in inefficient double strand break(DSB) repair by NHEJ (18). Interestingly, $\mathrm{SNF} 2 \mathrm{H}$ is also required for BRCA1 recruitment to break sites in a $\mathrm{pH} 2 \mathrm{AX}$ independent manner downstream of H2B ubiquitination by RNF20 (19). 
We have previously characterized SNF2H as a target of microRNA regulation (20).

microRNAs are small 17-25 nucleotide RNA molecules involved in post-transcriptional regulation of gene expression in metazoans in a sequence-specific manner (21). microRNAs in the same family (e.g. miR-99a, -99b and -100) have nearly identical sequence and target the same sets of mRNAs. microRNAs are crucial for development of higher organisms and each microRNA can regulate the expression of many proteins to enact complex changes in cellular phenotype. MicroRNAs have been found to be intimately involved in the differentiation of many tissue types during development (22-26). Additionally, in cancer, microRNAs are often misregulated, with certain miRNAs able to function as tumor suppressive and oncogenic factors (27-30).

In a screen for microRNAs that modulate a cell's response to DNA damage, we found that expression of members of the miR-99 family were upregulated following DNA damage and that miR-99 expression correlated with radiation sensitivity. The downregulation of SNF2H, a miR-99 family target, mediated radiation sensitivity through its role in facilitating DNA repair. The upregulation of the miR-99 family following radiation decreased the efficiency of repair factor recruitment and the rate of DNA repair after a second exposure to ionizing radiation. Thus, the induction of miR-99 represents a switch by which cells subjected to multiple rounds of radiation are directed away from continuing to repair their DNA. Interestingly, fractionation of radiation in radiotherapy is based on the principle that multiple smaller doses of radiation are more effective than a single large dose of radiation. Although fractionated therapy is widely used in clinical practice, molecular mechanisms underlying the differential radiosensitivity of some tumors to fractionated therapy are not entirely understood $(31,32)$. We suggest that induction of anti-DNA repair microRNAs such as the miR-99 family is one such molecular mechanism. 


\section{Results}

miR99 family members downregulated in radioresistant cancer sensitize cells to DNA damage by ionizing radiation

We were interested in examining microRNAs whose expression were misregulated in cancer and could alter the radiation sensitivity of cancer cells. We initially began by measuring differential microRNA expression in two breast cancer lines with differing radiation sensitivity. RNA was isolated from p53 positive MCF-7 and p53 negative SK-BR-3 cells before and 24 hours following treatment with 5 gy ionizing radiation(IR) and hybridized to Exiqon microarrays containing locked nucleic acid probes complementary to currently known microRNAs. We found 6 microRNAs to be more than 2 fold overexpressed in MCF7 cells and upregulated following irradiation (Fig. 1a). Among those upregulated were miR-21 and miR-26a, which are thought to be oncogenic microRNAs, targeting the PTEN tumor suppressor gene(33-35). miR-21* is also upregulated, but likely as an artifact of the increased expression of miR-21. Little is known about miR-105, though some evidence suggests it may be antiproliferative as it was found to reduce cyclin B expression in ovarian granulosa cells(36). The last two microRNAs among this group upregulated following irradiation and overexpressed in MCF7 were both members of the miR-99 family, miR-99a and miR-100. We had previously identified this family of microRNAs as being repressed during progression of prostate cancer and having a tumor suppressive role (20)(Fig. 1a). Their appearance in this screen suggested they may also play a role in altered radiation resistance during prostate and breast cancer progression.

To confirm the radiation sensitivities of the cancer cell lines differentially expressing miR-99 family members, we performed clonogenic assays following IR comparing the survival of MCF7 and SK-BR-3 cells, as well as LNCaP cells and their more advanced daughter cell line C4-2. We found SK-BR-3 cells to be significantly more resistant to radiation treatment than 
MCF7 cells (Fig. 1b), and C4-2 cells more resistant than LNCaP cells (Fig. 1e), correlating with lower basal expression of miR-99a and miR-100 as measured by qPCR (Fig. 1c, 1f). qPCR was used to confirm that miR-99 family members were upregulated following IR treatment. We found miR-99a and miR-100 to be 3-4 fold upregulated in MCF7 and LNCaP cells 24 hours following IR, when normalized to $\boldsymbol{\beta}$-actin, confirming the results of the microarray analysis, and suggesting their upregulation occurs following irradiation of prostate cancer cell lines as well

(Fig. 1d, 1g), similar results were observed when normalized to u6snRNA or GAPDH (Fig. S1a, S1b).

Having observed a correlation between decreased expression of the miR-99 family and radiation resistance, we wanted to determine whether reintroduction of miR-99 family members into cells could increase DNA damage sensitivity. We introduced exogenous miR-99a or miR100 into C4-2 and SK-BR-3 cells, treated with ionizing radiation and examined clonogenic survival. We found a significant reduction in survival following radiation in the presence of exogenous miR-99 family members (Fig. 2a, 2b). These data suggest that the miR-99 family may increase the sensitivity of cells to DNA damage and that a decrease in miR-99 family member expression may be a mechanism by which cancer cells can acquire resistance to DNA damaging agents.

\section{miR-99 and siRNA of its target SNF2H reduce BRCA1 localization to sites of DNA damage}

We previously identified the chromatin remodeling factor SNF2H as a direct target of miR-99a in LnCAP and C4-2 cells (20). SNF2H has recently been implicated in the repair of DNA damage by HR and NHEJ, and is an important member of complexes required for the recruitment and function of DNA repair proteins to sites of DSBs $(18,19)$. We were curious to see whether introduction of miR-99 family members would have an effect on DNA repair through SNF2H. We began by confirming that miR-99a and miR-100 could reduce the level of 
SNF2h in cells by performing western blots following transfection of double stranded mimic oligonucleotides. As expected, miR-99a and miR-100 significantly reduced the levels of SNF2H, though not to the same extent as siRNA targeting the 3' UTR of SNF2H (Fig. 2c). To determine if the miR-99 family could affect the recruitment of DNA proteins to sites of damage, we introduced miR-99a or siRNA targeting the 3' UTR of SNF2H into C4-2 cells and subjected them to gamma irradiation. We then examined BRCA1 and $\mathrm{pH} 2 \mathrm{AX}$ foci formation by immunofluorescence. miR-99a, miR-100 and siSNF2H greatly reduced the number of cells expressing intense BRCA1 ionizing radiation induced foci (Fig. 3a, S1C, S2). Neither treatment reduced $\mathrm{pH} 2 \mathrm{AX}$ foci following IR. We also examined $\mathrm{MDC} 1$ and conjugated ubiquitin foci formation following IR, and saw no significant reduction in the number of these foci (Fig. S3). This is consistent with the recent finding that SNF2H facilitates BRCA1 recruitment downstream of the ubiquitination of H2B by RNF20 in a pH2AX independent manner(19).

\section{Recruitment of DNA repair proteins is unaffected by miR99 in cells expressing non- targetable SNF2H}

To determine if repression of SNF2H by miR-99a was responsible for the repression of BRCA1 recruitment by miR-99a, we stably expressed the SNF2H ORF in C4-2 cells. The absence of the 3' UTR makes this form of SNF2H resistant to miR-99a and we examined

BRCA1 foci in these cells following irradiation. The non-targetable SNF2H rescued BRCA1 foci formation both after treatment with miR-99a as well as siRNA targeted to the SNF2 3'UTR (Fig. 3B, S1D). This evidence shows that miR-99a inhibits BRCA1 recruitment through the downregulation of SNF2H.

\section{miR-99 and siSNF2H reduce Rad51 recruitment to sites of DNA damage}

Since BRCA1 is contained within multiple protein complexes that can either promote or repress DNA repair by homologous recombination, we examined whether miR-99 could repress 
recruitment of proteins directly involved in HR. miR-99a or 100, and siSNF2h were introduced into C4-2 cells, and Rad51 foci formation was examined following irradiation. Both the miR-99

family as well as siSNF2H greatly reduced Rad51 foci formation (Fig. 3c, S4), suggesting the recruitment of this essential component of the HR pathway requires SNF2H, and can be blocked by overexpression of the miR-99 family.

\section{miR-99 reduces the rate of DNA repair through downregulation of SNF2H}

To assess the effect of miR-99a on the efficiency of DNA repair, we transfected C4-2 cells with miR-99a or siSNF2H and performed neutral agarose comet assays, which detect DSBs, following treatment with IR. With control siRNA oligo, the majority of double-strand DNA breaks was repaired within 60 minutes of treatment with IR. In the presence of miR-99a or siSNF2H, the DNA damage persisted, resulting in a 3 fold greater tail moment than the control at 60 minutes following irradiation (Fig. 4a, S5a). A similar effect was seen with introduction of miR-100 (Fig. S6) However when we stably expressed the SNF2H ORF in these cells ,the repression of DNA repair by miR-99a or siSNF2H was reversed (Fig. $\mathbf{4 b}$, S5b). Therefore, miR99a reduces double-strand break repair following IR through repression of SNF2H.

\section{Exogenous miR-99a increases the extent of apoptosis following IR}

To determine if the deficit in DNA repair experienced by cells with exogenous miR-99 leads to an increase in apoptosis in these cells, we examined PARP cleavage in cells transfected with miR-99a following treatment with IR. While C4-2 cells treated with control siRNA displayed no increase in the ratio of cleaved PARP 48 hours following IR, miR-99a transfected cells displayed a marked increase in PARP cleavage after treatment with 2 and 5gy of IR (Fig. 4c). This suggests that miR-99a can modulate cell survival following DNA damage, likely through its ability to decrease DSB repair. 
miR-99a, miR-100 and siSNF2H block homologous recombination

Having observed the effect of miR-99a and miR-100 on BRCA1 foci formation, we set forth to determine if the microRNAs reduced the rate of successful HR following DNA damage. miR-99a, miR-100 and siSNF2H, were transfected into DR13-9 HeLa cells, which contain two partial GFP cassettes containing I-Sce1 sites $(37,38)$. Successful HR after an I-Sce1 induced double-strand DNA break produces a functional GFP, whose expression can be measured by FACS. In the presence of miR-99a, miR-100 or siSNF2H, we observed a 40 percent reduction in HR activity as reported by the percentage of cells expressing GFP (Fig. 4d). By comparison, transfection with siRNA targeting ATM or BRCA1 resulted in 70 or 90 percent reductions in HR respectively. These data show that miR-99a, miR-100 and siSNF2H can reduce the efficiency of HR following double strand breaks, which correlates with the decreased recruitment of BRCA1 to sites of DNA damage.

\section{miR-99a, miR-100 and siSNF2H block efficient repair of double strand breaks by non- homologous end joining}

SNF2H is also important for the function of $\mathrm{Ku} 70 / 80$ at sites of DNA damage, facilitating efficient repair of double strand breaks by NHEJ(18). To determine if miR-99a and miR-100 could also reduce the efficiency of NHEJ we utilized a reporter assay that generates expression of DS-Red following successful NHEJ after I-Sce1-generated double strand breaks (39). siSNF2H caused a 60 percent reduction in NHEJ, represented by the percentage of DS-Red expressing cells. miR-99a or miR-100 produced a 50 percent reduction in NHEJ (Fig. 4e). siRNA targeting ATM also displays a significant reduction of NHEJ whereas siBRCA1 showed no significant reduction of NHEJ. This suggests that miR-99a/100 can also reduce the efficiency of repair of double strand breaks by NHEJ, as seen after repression of SNF2H. 
Induction of miR-99a and miR-100 following ionizing radiation reduces the efficiency of

DNA repair when confronted with a second round of radiation by blocking SNF2H

induction

Having observed that miR-99a and miR-100 are both induced following DNA damage and reduce the efficiency of DNA repair, we hypothesized that when confronted with multiple rounds of DNA damage, cells may become less efficient at DNA repair due to upregulation of the miR-99 family (Fig. 5a). We examined expression of the miR-99 family after radiation treatment over 24 hours and observed an increase in miR-99a and 100 expression of 3 fold over untreated cells (Fig. 5b). To determine if this induction resulted in an effect upon SNF2H expression, we examined SNF2H levels following irradiation in untreated cells, or in cells pretreated with IR 24 hours earlier. We found that in untreated cells, SNF2H is rapidly induced following radiation treatment, while in IR pretreated cells transfected with anti-GL2, this induction was no longer observed. However, when cells were treated with anti-99 (preventing the induction of miR99a or 100 (Fig. S6b), SNF2H was induced normally after the second radiation (Fig. 5d). Therefore the miR-99 induction after the first round of radiation prevents the upregulation of SNF2H protein following the second round of radiation. We also observed that SNF2H mRNA was transiently induced in all three treatment groups, indicating $\mathrm{SNF} 2 \mathrm{H}$ is transcriptionally upregulated following IR (Fig. S6c). However, the increased miR-99 in IRpretreated cells prevents the expected increase in SNF2H protein, as would be expected from the mechanism of action of a microRNA.

To test whether miR-99 induction, and prevention of SNF2H upregulation following IR had an effect on subsequent DNA repair, we performed a similar experiment and tested the efficiency of formation of DNA repair foci in untreated or IR pretreated cells. We found that after the first cycle of 2 gy IR, 80 percent of IR untreated cells formed strong BRCA1 foci. However, in IR pretreated cells transfected with anti-GL2, only 40 percent of cells formed strong 
BRCA1 foci after the second round of IR (Fig. 5d, S6a). In contrast, anti-99 treated cells had similar BRCA1 foci formation between IR untreated and pretreated cells, indicating that induction of the miR-99 family and subsequent inability to induce SNF2H is responsible for the reduction in BRCA1 recruitment to double strand break sites after a second round of DNA damage.

\section{Upregulation of miR-99 following DNA damage decreases the rate of DSB repair after a second round of radiation}

To examine whether upregulation of the miR-99 family following radiation could in fact slow the rate of DNA repair after a second round, we pretreated LNCaP cells with 2 gy of IR and performed neutral comet assays following a second 10 gy dose 24 hours later. During the time following pretreatment, cells were incubated with 2'-O-methyl antisense oligo inhibitors targeting either GL2(control) or the miR-99 family. Pretreatment with IR significantly increased DNA damage remaining 30 minutes following irradiation (Fig 6a, 6b, anti-GL2 pre). However, in cells containing anti-miR-99, there was no difference between the rate of repair of the pretreated and unpretreated cells, suggesting the decrease in repair following pre-irradiation of the control cells was due to miR-99 induction(Fig. 6a, 6b, anti-99 pre).

\section{Discussion}

We have identified that the miR-99 family is upregulated in response to ionizing radiation and reduces the ability of cells to repair damaged DNA. In light of the recent findings demonstrating SNF2H facilitates HR and NHEJ DSB repair $(18,19)$, we determined that miR99a could alter the efficiency of DNA repair by regulating the expression of SNF2H. Exposure to a single dose of DNA damage induces DNA repair pathways and also leads to increased miR-99 family expression. This subsequent induction of the miR-99 family prevents SNF2H from being rapidly induced following DNA damage, which decreases DNA repair efficiency if multiple 
rounds of DNA damage are experienced 24 hours apart. The expression of this microRNA is decreased in more advanced cancers, suggesting that the resultant greater DNA repair efficiency makes these cell lines more resistant to radiotherapy. Loss of this microRNA may represent a mechanism by which cancer cells acquire resistance to DNA damage by allowing efficient repair to continue after multiple mutagenic insults inflicted by radiotherapy. In addition, the observation that miR-99 expression correlates with p53 status suggests its induction also requires intact DNA damage checkpoint pathways, and the mechanism of this regulation warrants further study. The importance of the miR-99 family in regulating cellular response to DNA damage is further supported by recent findings that the miR-99 family targets pro-survival proteins IGF-1R and mTOR $(20,40)$, indicating that there are multiple pathways the miR-99 family targets that would presumably have an effect on cells survival following DNA damage.

microRNAs are regulated by the DNA damage response and alter the expression of many of the signaling and effector components of DNA repair (41). For example, the DNA damage signaling histone variant $\mathrm{H} 2 \mathrm{AX}$ is regulated by miR-24 in post mitotic hematopoetic cells, rendering them hypersensitive to DNA damaging agents (42). The DNA damage checkpoint protein Cdc25a is regulated by miR-21, which is upregulated following DNA damage, resulting in slowing of cell cycle progression (43). The p53 tumor suppressor upregulates the transcription of the miR-34a-c family, which in turn alters gene expression, slowing cell cycle progression (44). Additionally, microRNA biogenesis is regulated at the processing step that converts primary miR to precursor miR by the association of $\mathrm{p} 53$ with the Drosha/DGCR8 complex, resulting in upregulation of several growth suppressive microRNAs (45). BRCA1 is directly downregulated by miR-182, resulting in impaired HR and sensitivity to ionizing radiation (46). The targeting of SNF2H, a protein required both for efficient HR as well as NHEJ repair by the miR-99 family is an additional level of control that microRNAs can exert over the DNA damage response, decreasing double strand break repair throughout the cell cycle. It will be of interest to 
examine the mechanism of regulation of the miR-99 family, how its upregulation is p53 dependent, and what epigenetic changes lead to a decrease of its expression in more advanced cancers. Additionally, while it is clear that chromatin remodeling factors such as SNF2H are required for efficient recruitment of DDR proteins, it will be of great interest to determine exactly which steps in the response require chromatin remodeling activity, and how expression and recruitment of these complexes to sites of DNA damage are regulated to facilitate repair.

A matter of great curiosity is how the regulation of DSB repair efficiency by the upregulation of miR-99 following DNA damage interacts with the timing and efficacy of fractionated radiation therapy in the treatment of cancers. Fractionation of radiotherapy has been in use since the early $20^{\text {th }}$ century when it was found to be effective in maximizing damage to proliferating cells while minimizing damage to normal tissue. Fractionated therapy increases the therapeutic index of radiation by facilitating reoxygenation and cell cycle redistribution of rapidly dividing tumor cells while simultaneously allowing repair of sub-lethal DNA damage in non-dividing cells and repopulation of normal cells from the surrounding tissue. Recent attention has been focused on alternative regimens of fractionated therapy to increase their efficacy, as many protocols have been mostly unchanged since the 1930s (31). While altered regimens are being developed to minimize damage to surrounding tissues, consideration should be given to molecular mechanisms such as the induction of the miR-99 family to confer optimal tumor radiation sensitivity at given time points during treatment. Additionally, since decrease of miR99 family expression in more advanced cancer cells correlates with their radioresistance and transfection of these microRNAs reduced cell survival following radiation treatment, in-vivo reintroduction of the miR-99 may be useful as a sensitizer to radiation therapy once microRNA delivery to tumor cells becomes feasible. 


\section{Materials and Methods}

\section{Cell Culture}

Human prostate cancer cell lines LnCAP and C4-2, as well as human breast cancer cell lines MCF7 and SK-BR-3 were obtained from ATCC. HeLa DR13-9 cells $(37,38)$ were obtained from Dr. J.D. Parvin (Ohio State University). NHEJ-DSRed 293B cells were obtained from the Dr. J. Larner (UVA) and K. Valerie (VCU) (47). LnCAP and C4-2 were grown in RPMI 1640 medium supplemented with 10\% FBS. MCF7, HeLa DR13-9 and NHEJ-DSRed 293B cells were cultured in DMEM with 10\% FBS, and SK-BR-3 cells were cultured in McCoy’s 5a medium with 10\% FBS. All cell lines were cultured under normal conditions.

\section{Clonogenic assays}

Cells were transfected in $10 \mathrm{~cm}$ dishes with 20 picomoles of miR-99a duplex or siRNA against firefly luciferase as a control. The oligonucleotides were incubated for 30 minutes with 8 $\mathrm{uL}$ Lipofectamine RNAimax per plate in $2 \mathrm{~mL}$ Optimem before mixing with trypsinized cells and plated at a $20 \%$ confluence. Plates were incubated for 48 hours before seeding to 6-well plates in the presence of $20 \mathrm{nM}$ duplex oligo at increasing seeding densities corresponding with the planned dose of radiation. 6-well plates were irradiated in a Shepherd Mark 1 Cs-137 irradiator. Cells were incubated until colonies arising from single cells reached 25 cells or more, with media changed every 72 hours. Colonies were counted, and clonogenic survival was calculated by dividing the number of colonies at a given dose by the number of colonies in the un-irradiated control samples, and multiplied by the seeding density coefficient. 


\section{miRNA expression analysis}

RNA was isolated from cells using Trizol extraction (Invitrogen). For microarray analysis, RNA was further purified using RNAeasy RNA cleanup kit (Qiagen). For qPCR validation, Poly A tailing and cDNA preparation of mature microRNAs was performed using the NCODE miRNA amplification system (Invitrogen). qPCR amplification was performed using forward primers identical to the mature miRNA sequence, and NCODE universal reverse primers with Sybr Green ER (Invitrogen). Expression was normalized to u6snRNA, which used the primer sequence 5'-CTGCGCAAGGATGACACGCA-3'. The sequence of miR-99a is 5'aacccguagauccgaucuugug-3', and the sequence of miR-100 is 5'- aacccguagauccgaacuugug-3'.

\section{Immunofluorescence}

Cells were plated on glass cover slips in the presence of 20nM siRNA duplex. After 72 hours, the coverslips were irradiated with 5gy IR. After 60 minutes, the cover slips were fixed with 4\% formaldehyde in PBS, and permeabilized in 0.5\% Triton X-100 in PBS. Coverslips were blocked in 5\% goat serum, or 5\% donkey serum if primary antibody is of goat origin (MDC1). Coverslips were incubated at room temperature with primary antibody for 1 hour, and Alexa 488 or 549 conjugated secondary antibody for 1 hour, with three TBS washes following each antibody incubation. Coverslips were then mounted with Vectashield mounting solution (Vector Laboratories). Images were collected at equal exposures and foci were counted manually using ImageJ. Antibodies used were BRCA1 D-9(Santa Cruz), pH2AX (Cell signaling, Millipore), Rad51 H-92 (Santa Cruz) MDC1 C-20(Santa Cruz), Anti conjugated ubiquitin FK2 (Millipore). pH2AX primary antibodies was used at a 1:200 concentration in 5\% goat serum, MDC1 antibody was used at a 1:100 concentration in 5\% donkey serum, all other antibodies were used at a 1:100 concentration in 5\% goat serum. 


\section{Western Blotting}

Cells were lysed in IPH buffer, run on 10\%- SDS-PAGE gels and transferred to nitrocellulose membranes. Blocking was performed with 3\% BSA and membranes were incubated overnight with SNF2H antibody(Abcam) before washing and secondary antibody incubation and Incubation with Millipore Immobilon HRP substrate.

\section{Microscopy}

Fluorescence images were acquired on a Nikon Microphot SA upright microscope equipped with a Nikon NFX35 camera using SPOT imaging software(Diagnostic Instruments Inc.) and a Nikon PlanApo 60x oil objective lens. For each experiment, fluorescence images were acquired on the same day using the same exposure times, gamma, and gain between samples. Images were enhanced for brightness and contrast to the same extent within Photoshop.

\section{HR/NHEJ assay}

20\% confluent HeLa DR13-9 cells and NHEJ-DS-Red 293B cells were transfected with 20nM siRNA using Lipofectamine RNAimax in $10 \mathrm{~cm}$ plates. After 24 hours, each plate was transfected with 20 ug I-Sce1 plasmid with Lipofectamine 2000. After another 48 hours, cells were trypsinized, resuspended in media and analyzed by FACS for GFP or DS-Red expression.

\section{Comet Assay}

Cells were transfected for 72 hours with $20 \mathrm{nM}$ siRNA oligo in $6 \mathrm{~cm}$ plates, followed by 10 gy irradiation. Cells were then trypsinized following irradiation and resuspended in ice cold PBS, incubated on ice until all time points were collected. Neutral comet assays were then performed using the Trevigen Comet assay kit standard protocol.

\section{Acknowledgement.}


AM was supported by DOD BCRP predoctoral traineeship BC073568. DS was supported by

DOD PCRP predoctoral traineeship PC094499. This work was supported by P01CA104106 to

AD. We thank members of the Dutta Laboratory for their help and discussion. 


\section{References:}

1. van Attikum H, Gasser SM. Crosstalk between histone modifications during the DNA damage response. Trends in Cell Biology. 2009;19(5):207-17.

2. Celeste A, Petersen S, Romanienko PJ, Fernandez-Capetillo O, Chen HT, Sedelnikova OA, et al. Genomic Instability in Mice Lacking Histone H2AX. Science. 2002 May 3, 2002;296(5569):922-7.

3. van Attikum H, Gasser SM. The histone code at DNA breaks: a guide to repair? Nat Rev Mol Cell Biol. [10.1038/nrm1737]. 2005;6(10):757-65.

4. van Attikum H, Fritsch O, Hohn B, Gasser SM. Recruitment of the INO80 complex by H2A phosphorylation links ATP-dependent chromatin remodeling with DNA double-strand break repair. Cell. 2004 Dec 17;119(6):777-88.

5. van Attikum H, Fritsch O, Gasser SM. Distinct roles for SWR1 and INO80 chromatin remodeling complexes at chromosomal double-strand breaks. EMBO J.

[10.1038/sj.emboj.7601835]. 2007;26(18):4113-25.

6. Morrison AJ, Highland J, Krogan NJ, Arbel-Eden A, Greenblatt JF, Haber JE, et al. INO80 and [gamma]-H2AX Interaction Links ATP-Dependent Chromatin Remodeling to DNA Damage Repair. Cell. 2004;119(6):767-75.

7. Tsukuda T, Fleming AB, Nickoloff JA, Osley MA. Chromatin remodelling at a DNA double-strand break site in Saccharomyces cerevisiae. Nature. [10.1038/nature04148]. 2005;438(7066):379-83.

8. Lee H-S, Park J-H, Kim S-J, Kwon S-J, Kwon J. A cooperative activation loop among SWI/SNF, [gamma]-H2AX and $\mathrm{H} 3$ acetylation for DNA double-strand break repair. EMBO J. [10.1038/emboj.2010.27]. 2010;29(8):1434-45.

9. Jha S, Shibata E, Dutta A. Human Rvb1/Tip49 is required for the histone acetyltransferase activity of Tip60/NuA4 and for the downregulation of phosphorylation on H2AX after DNA damage. Mol Cell Biol. 2008 Apr;28(8):2690-700.

10. Stewart GS, Wang B, Bignell CR, Taylor AMR, Elledge SJ. MDC1 is a mediator of the mammalian DNA damage checkpoint. Nature. [10.1038/nature01446]. 2003;421(6926):961-6.

11. Mailand N, Bekker-Jensen S, Faustrup H, Melander F, Bartek J, Lukas C, et al. RNF8 Ubiquitylates Histones at DNA Double-Strand Breaks and Promotes Assembly of Repair Proteins. Cell. 2007;131(5):887-900.

12. Kolas NK, Chapman JR, Nakada S, Ylanko J, Chahwan R, Sweeney FdrD, et al. Orchestration of the DNA-Damage Response by the RNF8 Ubiquitin Ligase. Science. 2007 December 7, 2007;318(5856):1637-40.

13. Huen MSY, Grant R, Manke I, Minn K, Yu X, Yaffe MB, et al. RNF8 Transduces the DNA-Damage Signal via Histone Ubiquitylation and Checkpoint Protein Assembly. Cell. 2007;131(5):901-14.

14. Doil C, Mailand N, Bekker-Jensen S, Menard P, Larsen DH, Pepperkok R, et al. RNF168 Binds and Amplifies Ubiquitin Conjugates on Damaged Chromosomes to Allow Accumulation of Repair Proteins. Cell. 2009;136(3):435-46.

15. Roberts SA, Ramsden DA. Loading of the Nonhomologous End Joining Factor, Ku, on Protein-occluded DNA Ends. Journal of Biological Chemistry. 2007 April 6, 2007;282(14):10605-13.

16. Mladenov E, Iliakis G. Induction and repair of DNA double strand breaks: The increasing spectrum of non-homologous end joining pathways. Mutation Research/Fundamental and Molecular Mechanisms of Mutagenesis. 2011;711(1-2):61-72.

17. Larsen DH, Poinsignon C, Gudjonsson T, Dinant C, Payne MR, Hari FJ, et al. The chromatin-remodeling factor CHD4 coordinates signaling and repair after DNA damage. The Journal of Cell Biology. 2010 September 6, 2010;190(5):731-40. 
18. Lan L, Ui A, Nakajima S, Hatakeyama K, Hoshi M, Watanabe R, et al. The ACF1 Complex Is Required for DNA Double-Strand Break Repair in Human Cells. Molecular Cell. [doi: DOI: 10.1016/j.molcel.2010.12.003]. 2010;40(6):976-87.

19. Nakamura K, Kato A, Kobayashi J, Yanagihara H, Sakamoto S, Oliveira DNP, et al. Regulation of Homologous Recombination by RNF20-Dependent H2B Ubiquitination. Molecular Cell. [doi: DOI: 10.1016/j.molcel.2011.02.002]. 2011;41(5):515-28.

20. Sun D, Lee YS, Malhotra A, Kim HK, Matecic M, Evans C, et al. miR-99 Family of MicroRNAs Suppresses the Expression of Prostate-Specific Antigen and Prostate Cancer Cell Proliferation. Cancer Research. 2011;71(4):1313-24.

21. He L, Hannon GJ. MicroRNAs: small RNAs with a big role in gene regulation. Nat Rev Genet. 2004 Jul;5(7):522-31.

22. Dey BK, Gagan J, Dutta A. miR-206 and -486 Induce Myoblast Differentiation by Downregulating Pax7. Mol Cell Biol. 2011 January 1, 2011;31(1):203-14.

23. Gagan J, Dey BK, Layer R, Yan Z, Dutta A. MICRORNA-378 targets the myogenic repressor myor during myoblast differentiation. Journal of Biological Chemistry. 2011 April 6, 2011.

24. Bruno IG, Karam R, Huang L, Bhardwaj A, Lou CH, Shum EY, et al. Identification of a MicroRNA that Activates Gene Expression by Repressing Nonsense-Mediated RNA Decay. Molecular Cell. 2011;42(4):500-10.

25. Chen C-Z, Li L, Lodish HF, Bartel DP. MicroRNAs Modulate Hematopoietic Lineage Differentiation. Science. 2004 January 2, 2004;303(5654):83-6.

26. Houbaviy HB, Murray MF, Sharp PA. Embryonic Stem Cell-Specific MicroRNAs. Developmental Cell. 2003;5(2):351-8.

27. Lee YS, Dutta A. MicroRNAs in Cancer. Annual Review of Pathology: Mechanisms of Disease. 2009 2009/02/01;4(1):199-227.

28. Lee YS, Dutta A. The tumor suppressor microRNA let-7 represses the HMGA2 oncogene. Genes Dev. 2007 May 1;21(9):1025-30.

29. He L, Thomson JM, Hemann MT, Hernando-Monge E, Mu D, Goodson S, et al. A microRNA polycistron as a potential human oncogene. Nature. [10.1038/nature03552]. 2005;435(7043):828-33.

30. Chang T-C, Wentzel EA, Kent OA, Ramachandran K, Mullendore M, Lee KH, et al. Transactivation of miR-34a by p53 Broadly $†$ Influences Gene Expression and $†$ Promotes $†$ Apoptosis. Molecular Cell. 2007;26(5):745-52.

31. Marcu LG. Altered fractionation in radiotherapy: From radiobiological rationale to therapeutic gain. Cancer Treatment Reviews. 2010;36(8):606-14.

32. Skvortsova I, Skvortsov S, Stasyk T, Raju U, Popper B-A, Schiestl B, et al. Intracellular signaling pathways regulating radioresistance of human prostate carcinoma cells.

PROTEOMICS. 2008;8(21):4521-33.

33. Meng F, Henson R, Wehbe, ÄìJanek H, Ghoshal K, Jacob ST, Patel T. MicroRNA-21 Regulates Expression of the PTEN Tumor Suppressor Gene in Human Hepatocellular Cancer. Gastroenterology. 2007;133(2):647-58.

34. Huse JT, Brennan C, Hambardzumyan D, Wee B, Pena J, Rouhanifard SH, et al. The PTEN-regulating microRNA miR-26a is amplified in high-grade glioma and facilitates gliomagenesis in vivo. Genes \& Development. 2009 June 1, 2009;23(11):1327-37.

35. Chan JA, Krichevsky AM, Kosik KS. MicroRNA-21 Is an Antiapoptotic Factor in Human Glioblastoma Cells. Cancer Research. 2005 July 15, 2005;65(14):6029-33.

36. Sirotkin AV, Lauková M, Ovcharenko D, Brenaut P, Mlynček M. Identification of MicroRNAs controlling human ovarian cell proliferation and apoptosis. Journal of Cellular Physiology. 2010;223(1):49-56. 
37. Ransburgh DJR, Chiba N, Ishioka C, Toland AE, Parvin JD. Identification of Breast Tumor Mutations in BRCA1 That Abolish Its Function in Homologous DNA Recombination. Cancer Research. 2010 February 1, 2010;70(3):988-95.

38. Pierce AJ, Johnson RD, Thompson LH, Jasin M. XRCC3 promotes homology-directed repair of DNA damage in mammalian cells. Genes Dev. 1999 Oct 15;13(20):2633-8.

39. Golding SE, Morgan RN, Adams BR, Hawkins AJ, Povirk LF, Valerie K. Pro-survival AKT and ERK signaling from EGFR and mutant EGFRvIII enhances DNA double-strand break repair in human glioma cells. Cancer Biol Ther. 2009 Apr;8(8):730-8.

40. Doghman M, Wakil AE, Cardinaud B, Thomas E, Wang J, Zhao W, et al. Regulation of Insulin-like Growth Factor,Äìammalian Target of Rapamycin Signaling by MicroRNA in Childhood Adrenocortical Tumors. Cancer Research. 2010 June 1, 2010;70(11):4666-75. 41. Hu H, Gatti RA. MicroRNAs: new players in the DNA damage response. Journal of Molecular Cell Biology. 2011 June 1, 2011;3(3):151-8.

42. Lal A, Pan Y, Navarro F, Dykxhoorn DM, Moreau L, Meire E, et al. miR-24-mediated downregulation of H2AX suppresses DNA repair in terminally differentiated blood cells. Nat Struct Mol Biol. [10.1038/nsmb.1589]. 2009;16(5):492-8.

43. Wang P, Zou F, Zhang X, Li H, Dulak A, Tomko RJ, et al. microRNA-21 Negatively Regulates Cdc25A and Cell Cycle Progression in Colon Cancer Cells. Cancer Research. 2009 October 15, 2009;69(20):8157-65.

44. He L, He X, Lim LP, de Stanchina E, Xuan Z, Liang Y, et al. A microRNA component of the p53 tumour suppressor network. Nature. [10.1038/nature05939]. 2007;447(7148):1130-4. 45. Suzuki HI, Yamagata K, Sugimoto K, Iwamoto T, Kato S, Miyazono K. Modulation of microRNA processing by p53. Nature. [10.1038/nature08199]. 2009;460(7254):529-33.

46. Moskwa P, Buffa FM, Pan Y, Panchakshari R, Gottipati P, Muschel RJ, et al. miR-182Mediated Downregulation of BRCA1 Impacts DNA Repair and Sensitivity to PARP Inhibitors. Molecular Cell. 2011;41(2):210-20.

47. Golding SE, Rosenberg E, Valerie N, Hussaini I, Frigerio M, Cockcroft XF, et al. Improved ATM kinase inhibitor KU-60019 radiosensitizes glioma cells, compromises insulin, AKT and ERK prosurvival signaling, and inhibits migration and invasion. Mol Cancer Ther. 2009 Oct;8(10):2894-902. 
Figures:

Figure 1
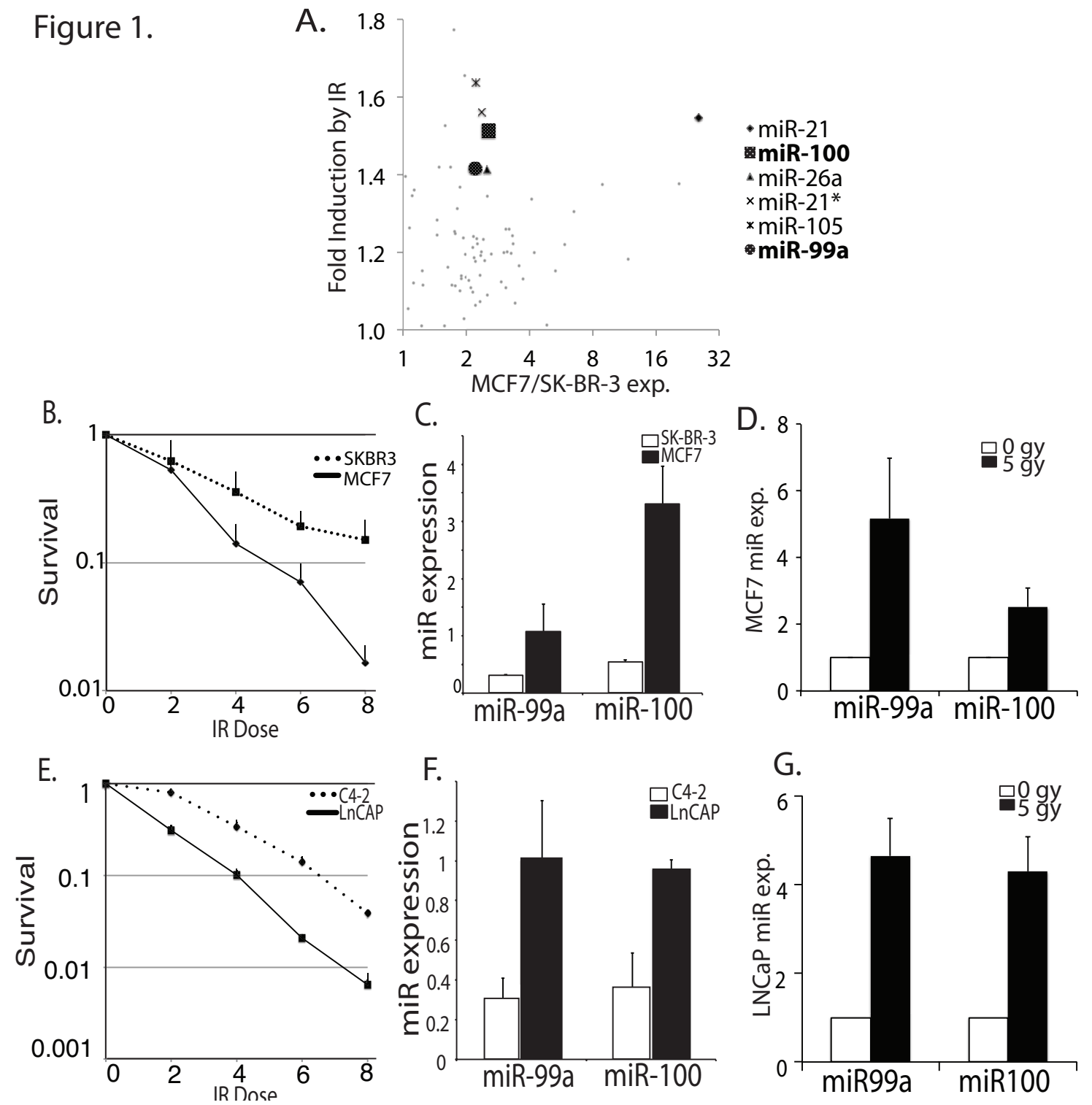

Figure 1. miR99 family members are downregulated in more radioresistant breast and prostate cancer cell lines and are upregulated following DNA damage. (A) Microarray screen of microRNAs induced by radiation. Y-axis: fold upregulated following irradiation in MCF7. Xaxis: fold expression in MCF7 (p53+) vs. SK-BR-3 (p53-). Highlighted microRNAs were at least 2 fold overexpressed in MCF7, and upregulated at least 1.4 fold 24 hours following treatment with IR. miR-99a and 100 were both found to meet these criteria. (B) Clonogenic assay of MCF7 and SK-BR-3 cells following IR. The colonies/well were counted to get fractional survival. Mean + SD ( $n=3)$. (C) miR-99a and miR-100 expression measured by qPCR in MCF7 and SKBR-3 cells, normalized to u6snRNA. Mean + SD ( $\mathrm{n}=6$ ). (D) miR-99a and miR-100 expression measured by qPCR, normalized to $\boldsymbol{\beta}$-Actin, 24 hours following IR treatment in MCF7 cells. Mean + SD (n=6). (E) Clonogenic assay of LNCap and C4-2 cells following IR. Fractional survival as in Fig. $1 \mathrm{~b}(\mathrm{n}=3)$. (F) miR-99a and miR-100 expression measured by qPCR in LNCaP and C4-2 cells, normalized to u6snRNA. Mean + SD (n=6). (G) miR-99a and miR-100 expression measured by qPCR, normalized to $\boldsymbol{\beta}$-Actin, 24 hours following IR treatment in $\mathrm{LNCaP}$ cells. Mean $+\mathrm{SD}(\mathrm{n}=6)$. 
A.

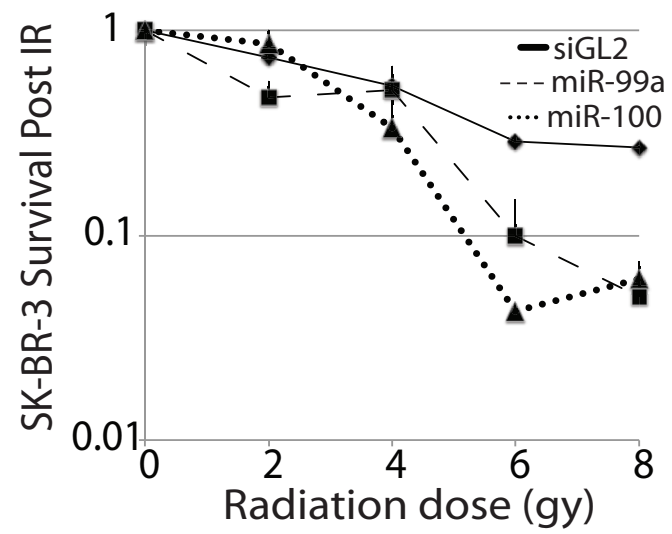

B.

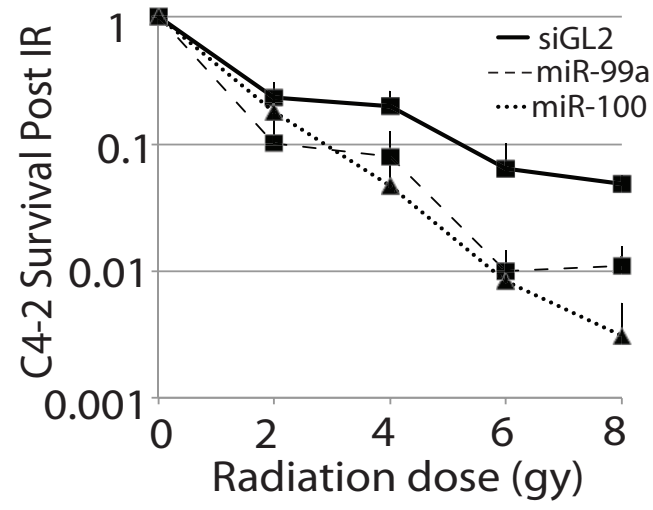

C.

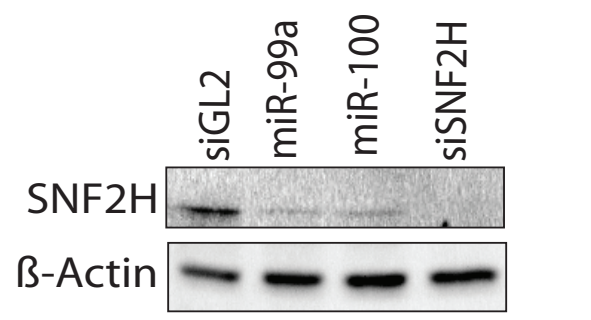

Figure 2. Introduction of exogenous miR-99a and miR-100 sensitize cells to ionizing radiation. (A) Clonogenic assay of SK-BR-3 cells treated with IR following transfection with double stranded mimics of miR-99a or miR-100. siGL2: negative control RNA-duplex targeting luciferase. Fractional survival as in Fig. 1b. (B) Clonogenic assay of C4-2 cells following transfection with double stranded mimics of miR-99a or miR-100. Fractional survival as in Fig. 1b. (C) Western blot for level of SNF2H in C4-2 cells following transfection with double stranded mimics of miR-99a or miR-100, or siRNA targeting SNF2H. 


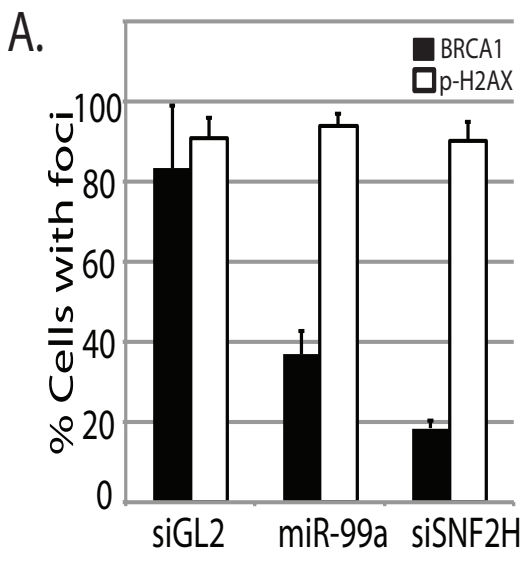

B.
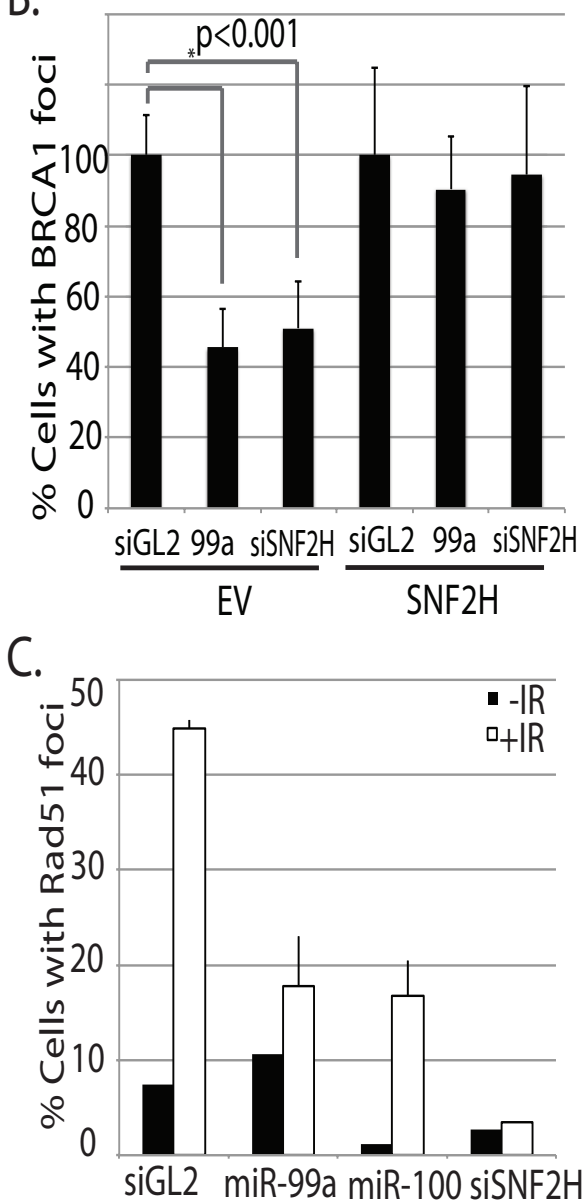

Figure 3. miR-99a reduces foci formation of BRCA1 and Rad51, but not $\gamma \mathrm{H} 2 \mathrm{AX}$ following IR. (A) Quantitation of BRCA1 and $\gamma \mathrm{H} 2 \mathrm{AX}$ immunofluorescence in C4-2 cells transfected with miR-99a or with siRNA targeting SNF2H 3' UTR $1 \mathrm{hr}$ following treatment with 5 gy IR. siGL2: negative control RNA duplex targeting luciferase. (B) Repression of BRCA1 foci is rescued by SNF2H. Quantitation of BRCA1 immunofluorescence in C4-2 cells transfected with miR-99a or siRNA targeting SNF2H 3' UTR $1 \mathrm{hr}$ following treatment with 5 gy IR. EV: Empty vector.

SNF2H: Cells expressing non-targetable SNF2H ORF. (C) Quantitation of Rad51 immunofluorescence in cells transfected with miR-99a, miR-100 or siRNA targeting SNF2H. Foci formation was examined 90min following treatment with 5 gy IR. Graphs show mean + SD, over 150 cells/sample. 


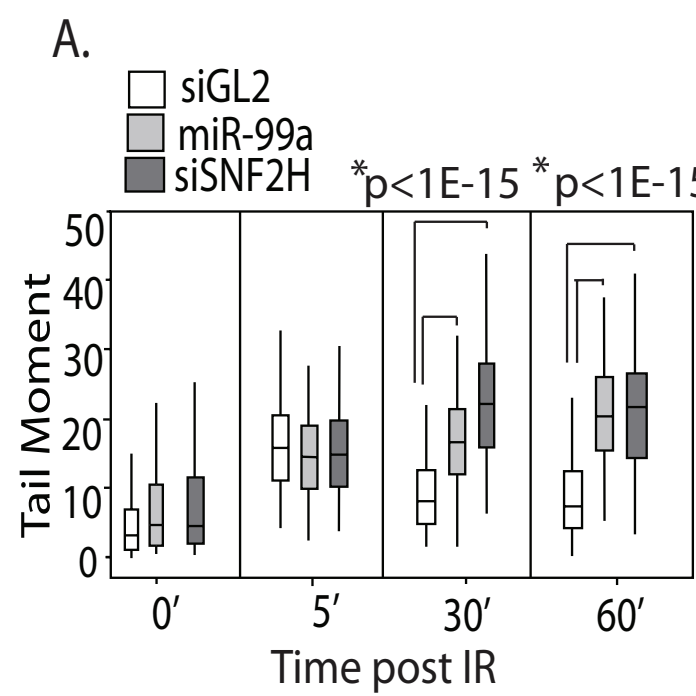

B.
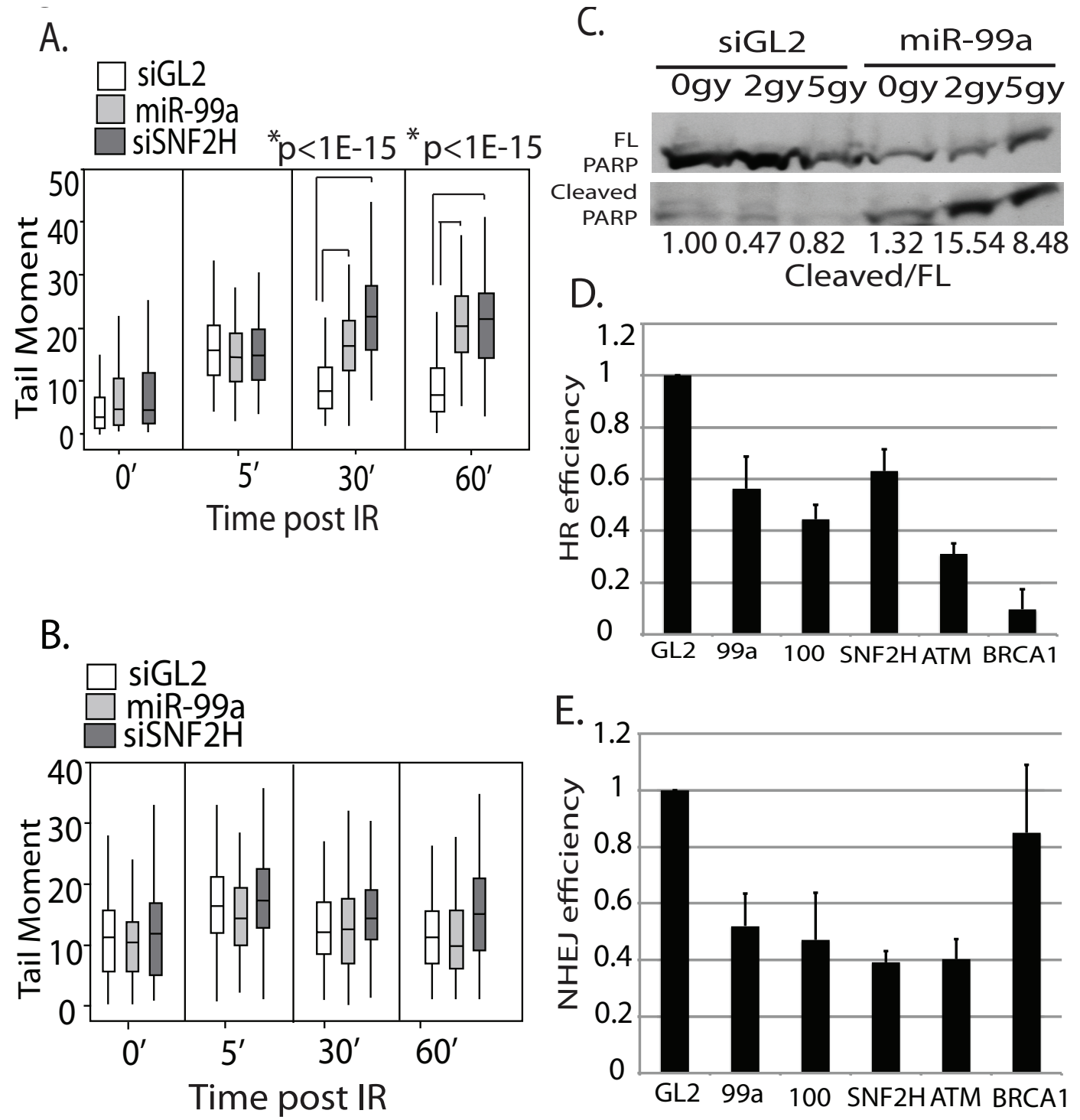

Figure 4. miR-99 slows the kinetics of DNA repair following IR through repression of SNF2H, increases apoptosis and reduces the efficiency of DNA repair by HR and NHEJ. (A) DNA damage was measured by neutral agarose gel comet assay at indicated times following 10 gy IR treatment of C4-2 cells infected with control PMSCV retrovirus. The cells were irradiated 72 hours after transfection with miR-99a or siSNF2H 3'UTR. Box plots representing quantitation of tail moment. Horizontal line: median. Rectangle: interquartile range. Vertical line: $5^{\text {th }}$ percentile to $95^{\text {th }}$ percentile. At least 100 comets per sample were analysed. (B) DNA damage was measured by neutral comet assay in cells stably expressing SNF2H without its 3'UTR, 72 hours after transfection with miR-99a or siSNF2H 3'UTR. Rest as in A. (C) Western blot showing full length and cleaved PARP 48 hours following radiation in cells transfected with control siRNA or miR99a. Normalized to full length PARP, Quantitation shown below. Full length PARP shown at a lighter exposure than cleaved PARP. (D) Percentage of GFP positive cells representing successful HR 48 hours following I-Sce1 transfection. DR13-9 HeLa cells were transfected with I-Scel after a 24 hour treatment with miR-99a, miR-100, siSNF2H, siATM, or siBRCA1. GL2: negative control duplex against luciferase. Mean $+\mathrm{SE}(\mathrm{n}=2)$. (E) Percentage of DS-Red positive cells representing successful NHEJ 48 hours following I-Sce1 induction. I-Sce1 was induced by doxycyline in 293B cells containing DS-Red-NHEJ reporter after a 24 hour treatment with miR99a, miR-100, siSNF2H, siATM, or siBRCA1. Mean + SD, over 150 cells/sample. 
A. 2 gy
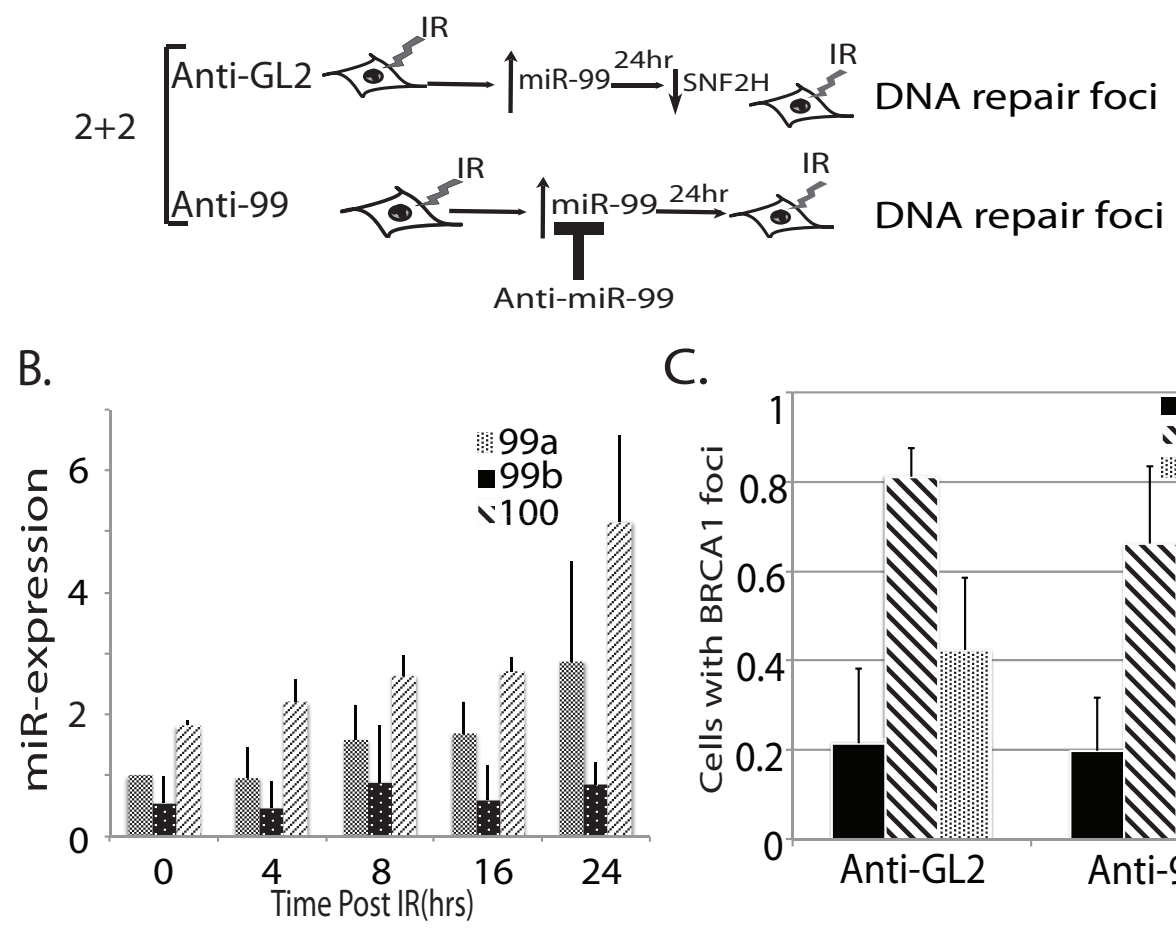

C.
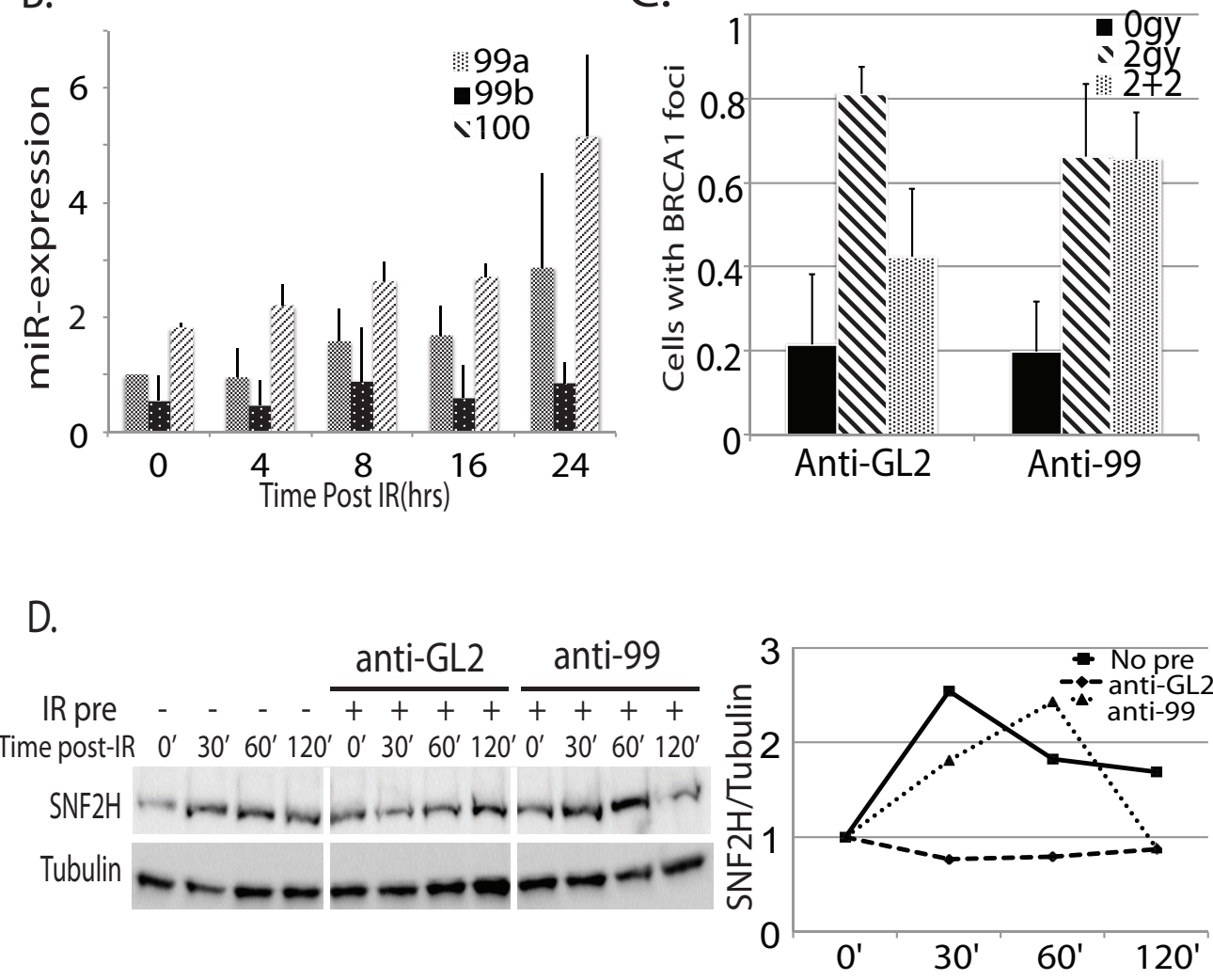

Figure 5. Induction of miR-99 family members following radiation in LnCAP cells decreases the efficiency of repair after subsequent rounds of radiation by preventing induction of SNF2H. (A) Schematic representation of the experiment in Fig. 5c. and 5d Cells were treated with 2 gy, incubated for 24 hours and then treated with another round of 2 gy IR. miR-99a/miR-100 induction was blocked by introduction of 2'-O-methyl antisense oligonucleotides targeting mir99a and miR-100 (anti-99) at the time of the first treatment (B) Expression of miR-99 family members by qRT-PCR over 24 hours following 2 gy IR. (C) Quantitation of BRCA foci formation in $\mathrm{LNCaP}$ with no radiation ( 0 gy) or conditions described above. At least 100 cells were counted in the irradiated samples and at least 45 in the unirradiated samples. $1: 100 \%$ of cells have $>10$ foci. Mean $+\mathrm{SD}$, over 150 cells counted/sample. (D) Western blot for SNF2H expression following 2 gy IR. Cells were left untreated or pretreated with 2 gy IR (IR pre) and incubated with 2'-O-methyl anti-GL2 or anti-miR-99 for 24 hours prior to second treatment of IR. Blots were quantitated and normalized to tubulin expression. Right: Expression relative to tubulin signal plotted relative to 0 ' time point. 

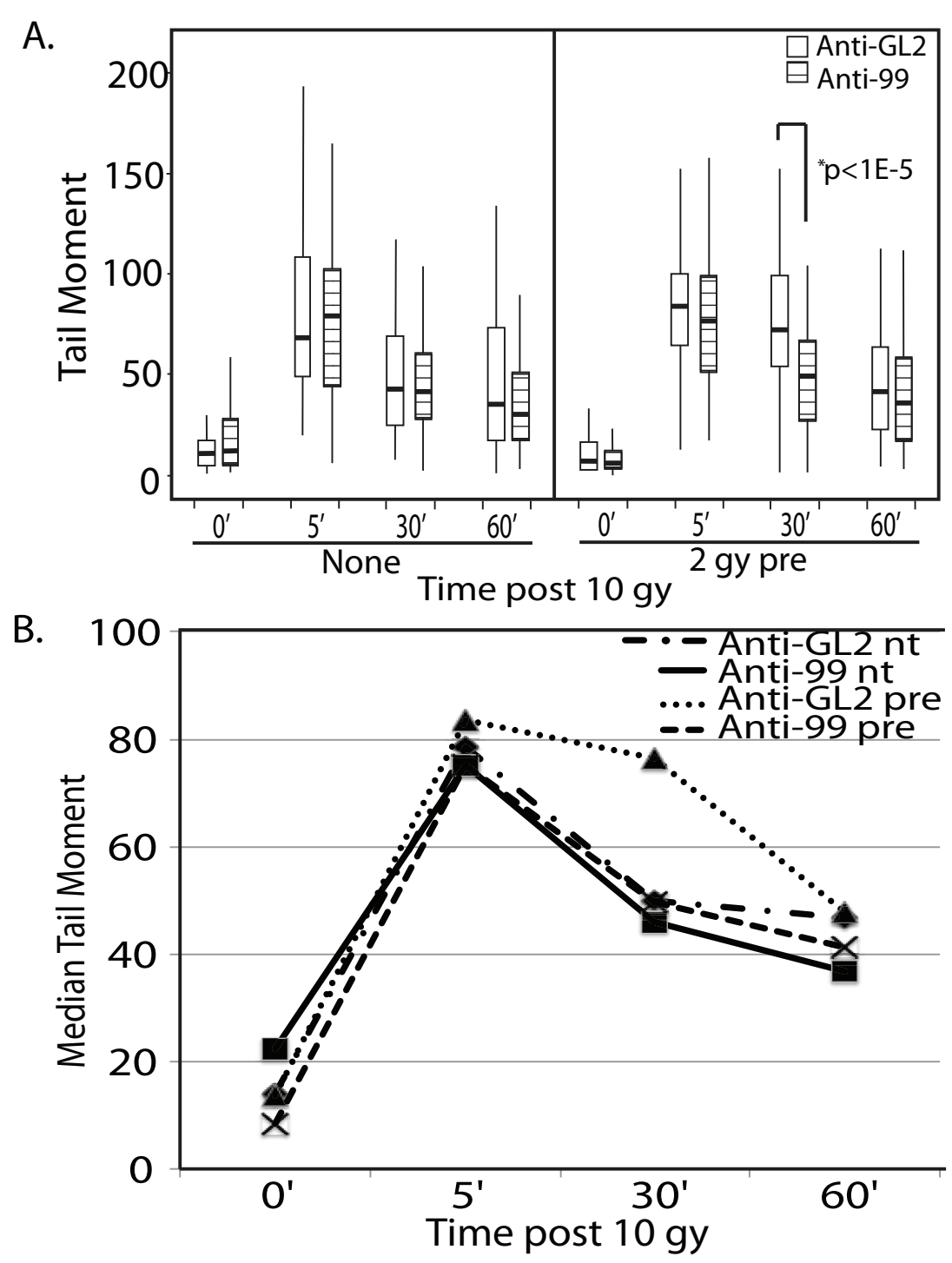

Figure 6. Induction of miR-99 family members following IR delays repair if exposed to a subsequent round of radiation. (A) Boxplot of comet tail moments observed in LNCaP cells. Cells were either left untreated (nt) or exposed to a 2 gy pretreatment of IR (pre). After 24 hours treatment with 2'-O-methyl antisense oligonucleotides targeting either GL2 or miR-99a and miR-100 (anti-99), the cells were exposed to 10 gy. DNA damage was examined by comet assay at the given time points. (B) Plot of the median tail moment of each treatment group in the experiment shown in panel A. At least 100 comets per sample were analyzed. 
A.

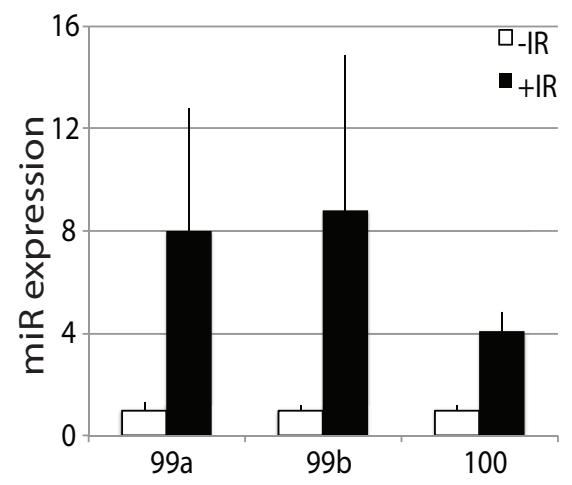

C.

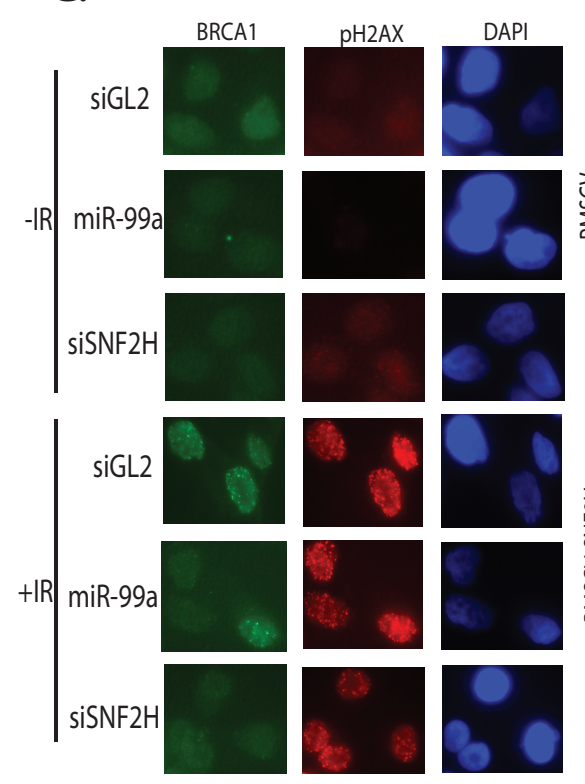

B.

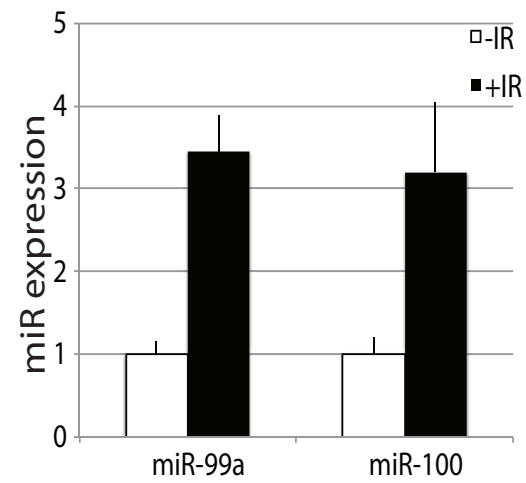

D.

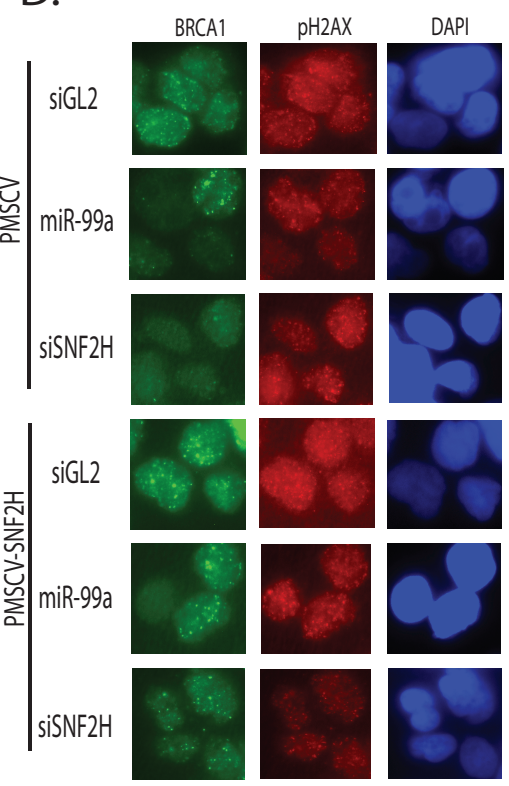

Supplemental Figure 1. miR-99 family induction following IR, and miR-99a and siSNF2H reduce BRCA1 foci by reducing SNF2H. (A) miR-99a, miR-99b, and miR-100 expression measured by qPCR, normalized to $\beta$-u6snRNA, 24 hours following IR treatment in MCF7 cells. Mean $+\mathrm{SD}(\mathrm{n}=6)(\mathbf{B})$ miR-99a and miR-100 expression measured by qPCR, normalized to GAPDH, 24 hours following IR treatment in LNCaP cells. Mean + SD (n=3). (C) Immunofluorescence showing BRCA1 foci following 5 gy irradiation in C4-2 cells transfected with siGL2, miR-99a, or siSNF2H. (D) Immunofluorescence showing BRCA1 and $\gamma \mathrm{H} 2 \mathrm{AX}$ foci $1 \mathrm{hr}$ following 0 gy (-IR) or 5 gy (+IR) irradiation in C4-2 cells infected with control PMSCV virus, or virus expressing the SNF2H ORF. The cells were transfected with siGL2, miR-99a or siRNA targeting the 3' UTR of SNF2H. 


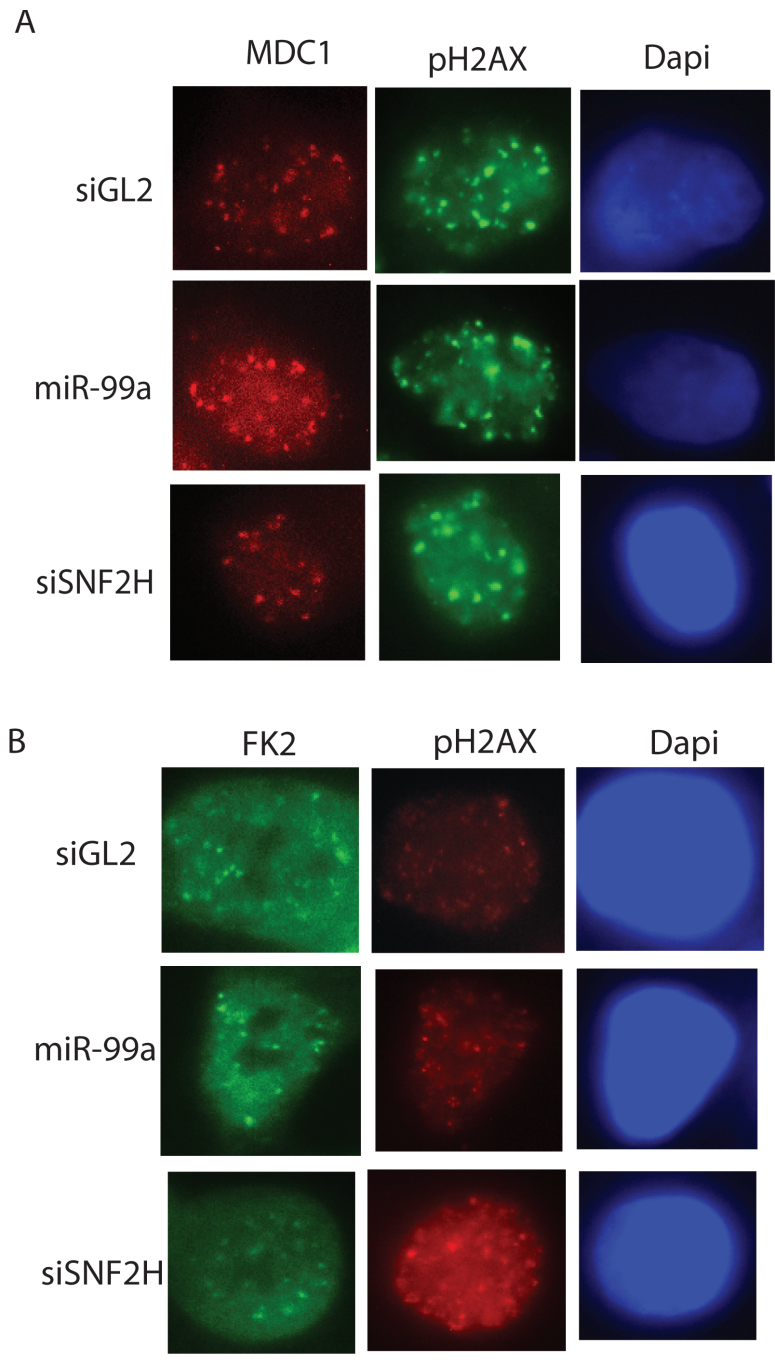

Supplemental Figure 2. miR-100 reduces BRCA1 foci formation following IR. (A) Immunofluorescence showing BRCA1 foci following 5 gy irradiation in C4-2 cells transfected with miR-100 or siRNA targeting GL2. (B) Quantitation of panel A. At least 75 cells were counted per sample. Mean $+\mathrm{SE}(\mathrm{n}=2)$. 

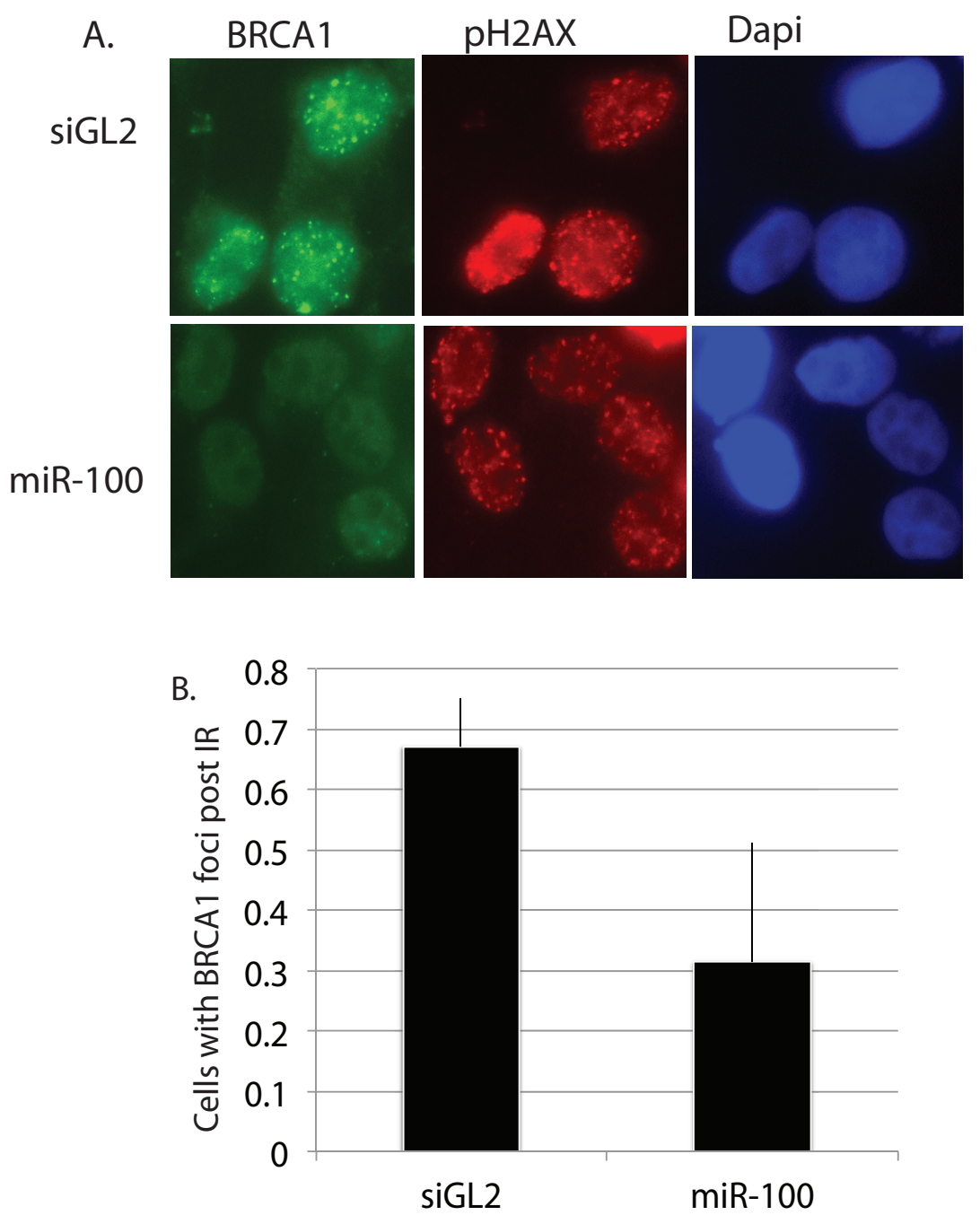

Supplemental Figure 3. miR-99a and siRNA targeting SNF2H do not reduce foci containing MDC1 or conjugated ubiquitin. (A) Immunofluorescence showing MDC1 foci following 5 gy irradiation in C4-2 cells transfected with siRNA targeting GL2, SNF2H, or a double stranded mimic of miR-99a. (B) Immunofluorescence showing conjugated ubiquitin foci using FK2 antibody following 5 gy irradiation in C4-2 cells transfected with siRNA targeting GL2, SNF2H, or a double stranded mimic of miR-99a. 


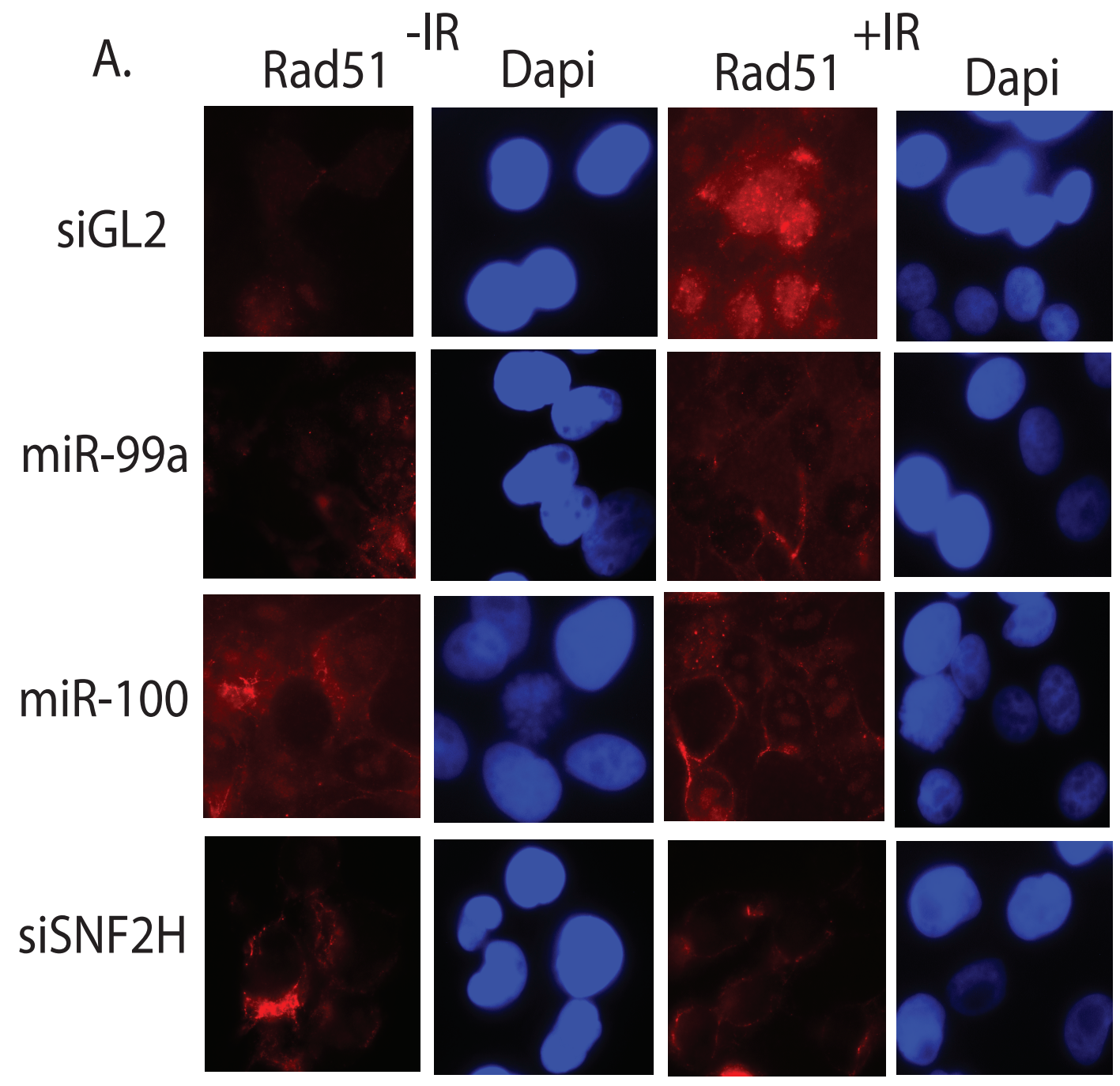

Supplemental Figure 4. The miR-99 family and siSNF2H reduce Rad51 foci formation following ionizing radiation. Immunofluorescence showing Rad51 foci formation 90 minutes following 0 gy or 5 gy irradiation in C4-2 cells transfected with siGL2, miR-99a, miR-100, or siSNF2H. 

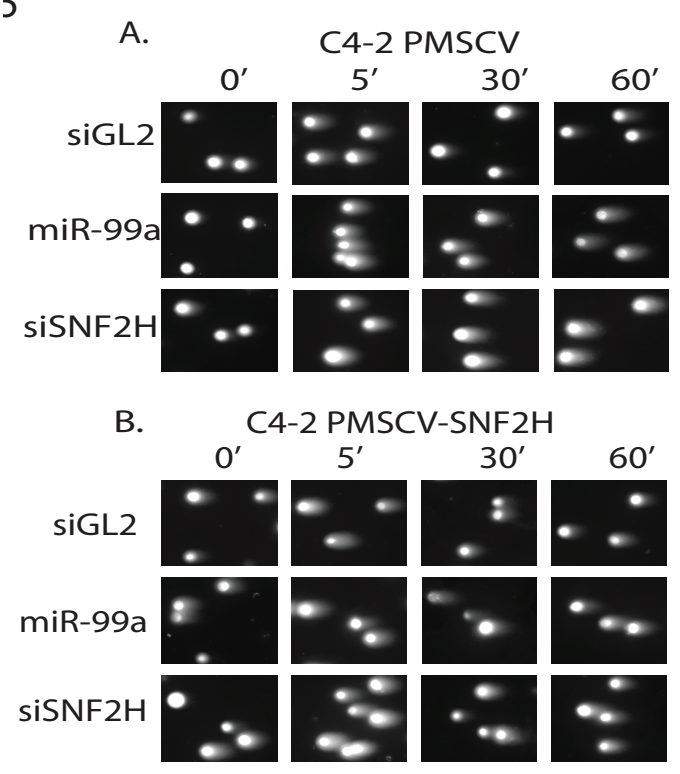

C.

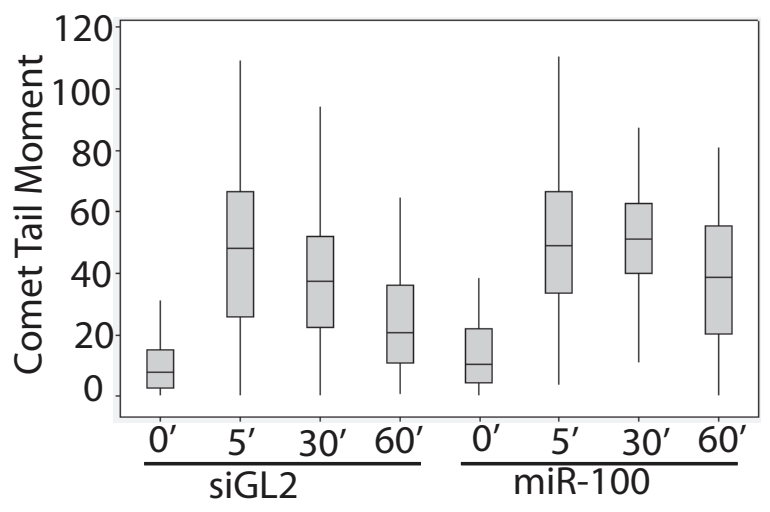

Supplemental Figure 5. miR-99a, miR-100 and SNF2H reduce the rate of DNA repair following ionizing radiation. (A) Neutral comet assays of control PMSCV infected C4-2 cells transfected with siGL2, miR-99a, or siRNA targeting the 3'UTR of SNF2H. Cells were treated with 10 gy IR and collected at the indicated time points. (B) Neutral comet assays of C4-2 cells infected with PMSCV virus expressing the SNF2H ORF and transfected with siGL2, miR-99a, or siRNA targeting the 3'UTR of SNF2H. Cells were treated with 10 gy IR and collected at the indicated time points. (C) miR-100 slows DSB repair in C4-2 cells. Quantitation of comet assays performed in C4-2 cells transfected with siRNA targeting GL2 or a double stranded mimic of miR-100. $\mathrm{X}$ axis indicates time following treatment with 10 gy of IR. 
A.

Anti-GL2

Anti-99
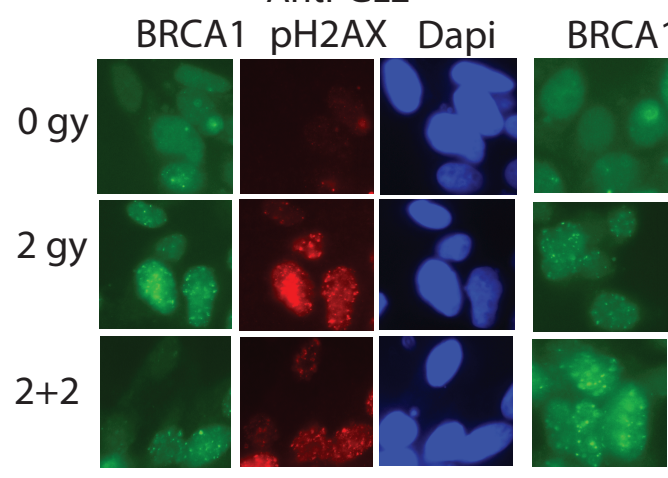

\section{Anti-g9}

$1 \mathrm{pH} 2 \mathrm{AX}$ Dapi

B.

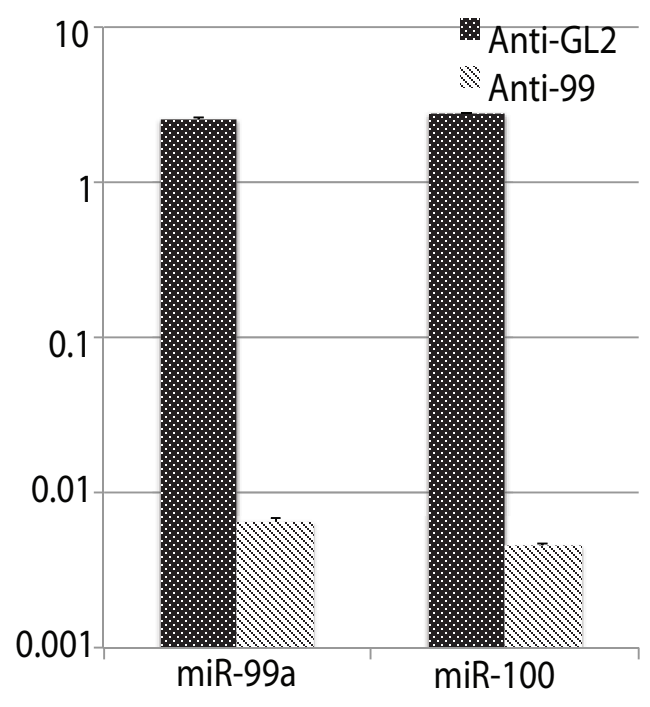

C.

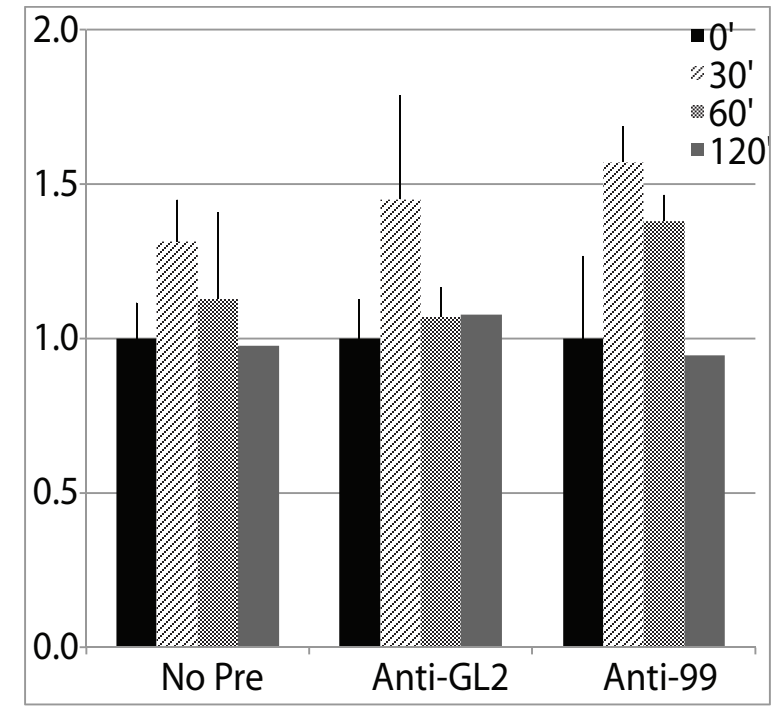

Supplemental Figure 6. Induction of miR-99 family following irradiation impairs DNA repair foci formation when facing subsequent DNA damage. (A) Immunofluorescence showing BRCA1 and pH2AX foci formation in C4-2 cells treated with 2'-O-methyl anti-GL2 or 2'-Omethyl anti-99 when unirradiated, treated with 2 gy, or treated with 2 gy, incubated for 24 hours and treated once more with 2 gy IR. (B) Reduction in miR-99a and miR-100 by 2'-O-methyl anti-99. qPCR analysis of miR-99a and miR-100 levels in LNCaP cells following transfection with 2'-O-methyl anti-miR-99. Mean + SD of 3 measurements. (C) qRT-PCR of SNF2H expression normalized to GAPDH in LnCAP cells either left untreated(no pre), or pretreated with 2 gy IR and incubated with 2'-O-methyl antisense oligonucleotides targeting GL2 negative control(anti-GL2) or miR-99a and miR-100(anti-99) for 24 hours prior to subsequent treatment with 2 gy IR. 


\title{
Chapter 3
}

\section{Adapted from: MUNC: A IncRNA that facilitates the function of MyoD in skeletal myogenesis}

Adam C. Mueller, Magdalena Cichewicz, Ryan Layer, Jeffrey Gagan, Anindya Dutta

In preparation

\begin{abstract}
:
High throughput sequencing of the transcriptome has led to the realization that a much larger proportion of the genome is expressed than previously thought. This has resulted in the discovery of numerous long-noncoding-RNAs (lncRNAs): a diverse class of widely expressed molecules that are important in a wide range of cellular processes and developmental contexts. In an in-silico screen for myogenic lncRNAs, confirmed by qPCR, we discovered several that are upregulated in myotubes versus myoblasts. One of these lncRNAs, which demonstrated high skeletal muscle specificity, was encoded $5 \mathrm{~kb}$ upstream of $M y o D$, a master transcription factor driving myogenesis, overlapping with a previously characterized enhancer termed the Distal Regulatory Region (DRR). RNAi of this lncRNA, which we named MyoD Upstream NonCoding (MUNC), reduced myoblast differentiation as measured by several markers of myogenesis. The myogenic defect in MUNC-depleted cells was accompanied by a reduction in MyoD binding at the MyoD Core Enhancer Region, Distal Regulatory Region and myogenin promoter. Enhancer activity of one of these regions was dependent on the presence of MUNC in myoblasts. Finally pulldown of MUNC using an array of complementary biotinylated oligonucleotides captured chromatin from the CER, DRR and myogenin promoter. All together this suggests that MUNC facilitates MyoD binding to its own promoter, as well as to other
\end{abstract}


MyoD target loci to facilitate its transcriptional activity and efficient myogenesis. This

mechanism suggests that other non-traditional enhancer elements in the genome may act both in cis and in trans through a non-coding RNA mediated pathway, rather than by simply recruiting transcription factors to their DNA sequence.

\section{Contribution:}

Adam Mueller designed and performed all of the experiments within the main figures aside from the bioinformatics screening. Madalena Cichewicz performed PCR walking experiments for MUNC characterization and assisted in the qPCR screen. Ryan Layer peformed bioinformatic lncRNA predictions. Jeffrey Gagan contributed to the design of the bioinformatic lncRNA screen. Anindya Dutta supervised all aspects of the study. 


\title{
MUNC: A IncRNA that facilitates the function of MyoD in skeletal myogenesis
}

\author{
Adam C. Mueller ${ }^{1}$, Magdalena Cichewicz ${ }^{1}$, Ryan Layer ${ }^{1}$, Jeffrey Gagan $^{1}$, Anindya Dutta ${ }^{1}$ * \\ ${ }^{1}$ Department of Biochemistry and Molecular Genetics, University of Virginia School of \\ Medicine, Jordan Hall 1232, Charlottesville, Virginia 22908 \\ *Corresponding Author
}

\section{Body:}

LncRNAs are rapidly becoming recognized as important regulators of gene expression in development and disease $(1,2)$. Our genomes undergo widespread transcription(3), and lncRNA loci are being discovered in comparable abundance to genomic loci encoding proteins(4). While lncRNAs are not as well conserved by comparison to protein coding genes(5), there have been a number of examples of lncRNAs that confer marked cellular and developmental phenotypes when their expression is altered by experiment or disease(6-10). We were interested in identifying functional lncRNAs in skeletal muscle development. Skeletal myogenesis is an ordered process whereby activation of $\mathrm{MyoD}$ in Pax3/MyoD expressing skeletal myoblasts results in a cascade of gene expression changes that results in terminal differentiation into multinucleated myotubes and myofibers $(11,12)$. The key transcriptional players in skeletal myogenesis are well known, but the mechanisms of their activation are not fully understood. MicroRNAs have been found to play significant roles in myogenesis at many key points, and are integral to regulating the gene expression changes that occur during terminal differentiation(13). However we currently have a limited understanding of the role lncRNAs play in this process. There have been examples of lncRNAs acting to repress transcription and mediate epigenetic silencing $(9,14,15)$, as well as acting as cis enhancers(16). Categorizing lncRNAs into classes 
with common roles is not yet possible by sequence analysis, and anyway, many other regulatory classes likely exist. With the advent of widely available public databases of genome wide histone modification, transcription factor and RNA expression data, and the development lncRNA prediction models(5), it is now possible to predict lncRNAs in a variety of developmental contexts in-silico. Using some of these available data sets, we predicted a number of lncRNAs whose expression was activated during skeletal myogenesis. Further examining these novel transcripts yielded an lncRNA of particular interest located adjacent to MyoD in the genome, which appears to facilitate MyoD's role in initiating the myogenic cascade in response to developmental signaling or injury.

We intersected publicly available databases showing where the mouse myoblast and myotube genomes are enriched in Histone H3K4 trimethylation marks, RNA polymerase II (17) and RNA transcripts (18), to identify myotube specific transcripts. From this set were removed all sequences containing known exons, and any sequences within 2 kilobases from known transcriptional start sites to predict potential myotube specific lncRNAs(Figure 1a). 29 putative myotube specific lncRNAs were identified (Supplemental Table 1), whose expression we then examined in $\mathrm{C} 2 \mathrm{C} 12$ myotubes and myoblasts using qRT-PCR. We found 9 of these 29 sequences to be more than 10 fold upregulated in myotubes (Figure 1b). Several of these sequences were located in genomic proximity to protein coding genes of interest in skeletal muscle differentiation. Over-expression of MyoD facilitates the trans-differentiation of 10T1/2 fibroblasts, and we found that this trans-diffferentiation was also accompanied by the induction of several of these lncRNAs (Figure 1c). In a time course experiment during $\mathrm{C} 2 \mathrm{C} 12$ differentiation, three potential lncRNAs were rapidly upregulated on the first day, approaching peak expression between day 2 and 3 of differentiation (Figure 1d).

Two of these lncRNA candidates were of particular interest in that they were located upstream of the genomic locus of the MyoD transcription factor, a master regulator of skeletal 
muscle differentiation (11). 5' Cap capture RACE PCR confirmed these two candidates to actually be a single spliced lncRNA (Supplemental figure 1a), and found its transcriptional start site to be located within a previously known MyoD enhancer element, known as the Distal Regulatory Region (DRR) (Figure 1f). 3' RACE PCR using oligo dT priming was unable to amplify a product at this locus, suggesting the mature lncRNA was not polyadenylated. Using random priming and PCR walking, we identified the 3' end of the transcript (Supplemental figure $1 \mathrm{~b}$ ), revealing a 518 b lncRNA composed of two exons separated by a 577 b intron (Figure 1f, Supplemental figure 1c). We have termed this lncRNA MUNC, for MyoD Upstream NonCoding. The Coding Potential Calculator(19) tool predicted this transcript to be in fact a long non-coding RNA with low coding potential or low evolutionary conservation in any of its short open reading frames(Supplemental figure 2a). Polysome fractionation found very little of the spliced or unspliced primary MUNC transcript in the polyribosome containing fraction, as compared to mRNA for proteins MyoD or GAPDH (Supplemental figure 2b).

Examining expression of several lncRNA candidates across mouse tissues (Supplemental figure 3a-d), we found MUNC to have the greatest specificity of expression for skeletal muscle, being upregulated during embryogenesis and having 600 fold greater expression in skeletal muscle than day 7 embryo (Figure 1e). Both the spliced mature MUNC and an unspliced isoform (Figure 1g) were upregulated during differentiation of primary myoblasts into myotubes, indicating MUNC is transcriptionally activated during myogenesis.

The overlap between MUNC and the MyoD distal regulatory region (DRR) brought up the possibility that the DRR enhancer acted through the expression of the lncRNA. The MyoD DRR is of interest in that it is dispensable for MyoD expression during embryogenesis, but is required for MyoD expression in adult satellite cells $(20,21)$. Interestingly, this parallels the requirement of MyoD for myogenesis: Myf5 can compensate for MyoD loss during embryonic development $(22,23)$, but adult satellite cells cannot efficiently regenerate muscle tissue after 
injury in the absence of $\mathrm{MyoD}(24)$. This suggests that the DRR-encoded MUNC, like MyoD, is required for myogenesis during regeneration in adult muscle and that MUNC is required to facilitate MyoD function by contributing to positive feedback of its expression in satellite cells.

Two independent siRNA duplexes targeting MUNC suppressed the upregulation of MUNC over the course of differentiation (Figure 2a). Northern blot analysis shows MUNC to be present in the nucleus and cytoplasm, but predominantly nuclear upon induction during differentiation (Supplemental figure 4a). MUNC knockdown significantly repressed the mRNA levels of myogenic markers upregulated during differentiation. The mRNA for Myogenin, an early marker of muscle differentiation and a transcription factor essential for muscle differentiation, and for Myosin Heavy Chain, a marker of terminal differentiation were both decreased by at least 5-fold on all the days of differentiation examined (Figure 2b-c). MyoD mRNA was repressed to a lesser degree: a 2-fold repression on days 1 and 3 (Figure 2d). Similarly when we examined protein expression, we found MHC was significantly repressed upon MUNC knockdown. Myogenin protein similarly had reduced expression at all days examined, while MyoD protein was reduced to a more modest extent (Figure 2e). The cell morphology of differentiating myoblasts showed less elongation and fewer nuclei per cell (Figure 2f). Immunofluorescence for MHC showed fewer MHC-positive multinucleate myotubes when MUNC was decreased (Figure $2 \mathrm{~g}$ ), as evidenced by a large reduction in fusion index (Figure 2h). There was a comparatively small decrease in the number of MyoD positive cells. A global analysis of gene expression in $\mathrm{C} 2 \mathrm{C} 12$ cells during differentiation found a much higher correlation between gene expression in growth medium versus differentiation media in cells where MUNC has been knocked down (Supplemental figure 5a-c) confirming the impaired differentiation. Genes that are most upregulated or downregulated during differentiation show a significant attenuation in the fold change in the cells where MUNC is knocked down 
(Supplemental figure 5d-e), confirming that MUNC is required for the global changes in gene expression during differentiation.

To gain insight into the mechanism by which MUNC facilitates muscle differentiation, we examined MyoD ChIP at its own regulatory regions: the 20kb upstream Core Enhancer Region, and the $5 \mathrm{~kb}$ upstream DRR, as well as the MyoD binding site in the myogenin promoter. Under control siRNA conditions, MyoD binding to each of these 3 loci was stimulated in differentiation medium. With MUNC knockdown however, MyoD binding was no longer stimulated, suggesting MUNC upregulation was necessary for MyoD recruitment to these target sites (Figure 3a-c). In addition, immunoprecipitation of MyoD and purification of associated RNA shows MUNC to interact with MyoD, suggesting that MUNC stimulates MyoD recruitment to target sites in the DNA likely through direct binding of MUNC with MyoD (Figure 3d).

To examine whether MUNC itself associates with regions of the chromatin where MyoD is localized, we precipitated chromatin by affinity to a pool of biotinylated oligonucleotides with sequence complementarity to MUNC, as detailed in(25). Q-PCR of select regions of the genome in the precipitates indicate that MUNC to associates at the Core Enhancer Region of MyoD under growth conditions, which is abolished by MUNC knockdown(Figure 3e). Under differentiation conditions, we found MUNC to also associate at the DRR of $M y o D$ and to the MyoD binding site in the Myogenin promoter. All these sites (CER, DRR and Myogenin promoter) associate specifically with MyoD protein indicating that MUNC RNA is recruited along with MyoD protein to these enhancer regions(Figure 3f).

To assay whether exogenous expression of MUNC co-activates MyoD in trans, we examined the effect of MUNC on MyoD's ability to stimulate luciferase expression driven by MyoD responsive enhancer elements. MUNC stimulated enhancer activity of the MyoD-CER 
by 2 fold, while it did not stimulate that of the DRR, MCK enhancer or a Mef $2 \mathrm{C}$ responsive region (Figure $3 \mathrm{~g}$ ). The experiment gave equivocal results in the face of our preceding result that MUNC stimulates MyoD binding to CER, DRR and the Myogenin promoter (Figure 3a-c) and that MUNC associates with all three sites (Figure 3f). One possibility is that although MUNC appears to bind with MyoD protein at all three sites, MUNC can stimulate MyoD activity only at a subset of sites. An alternative possibility is that the experiment in Figure $3 \mathrm{~g}$ has to be optimized to detect the effect of MUNC at all the MyoD-responsive promoters examined.

With the advent of high throughput sequencing, there is an ever-increasing volume of publicly available data that can be utilized to answer new research questions. Repurposing and intersecting three published datasets, we were able to identify a set of lncRNAs whose expression is upregulated during myogenesis. Within this set we discovered a noncoding RNA produced from a previously characterized MyoD enhancer. This DRR enhancer is required for MyoD expression in adult skeletal satellite cells, the physiologic context where MyoD is required for efficient myogenesis $(20,24)$. Previous studies found that this enhancer element requires integration into the chromosome to facilitate muscle specific transcription(26). Our findings suggest that this is likely due to the fact that this enhancer actually encodes a lncRNA which facilitates MyoD transcriptional activity, thereby promoting the expression of both MyoD and MUNC itself through binding to the DRR and the CER elements. This effect also appears to be specific to particular promoters, as MyoD driven transcription is not increased at all enhancers it is known to bind, suggesting MUNC may be altering the specificity of MyoD binding and transcriptional activity. This may represent a novel mechanism by which previously characterized noncanonical enhancer elements of transcription factors actually facilitate their target's transcription through a positive feedback loop using a RNA to activate their target gene's product. 


\section{Materials and Methods}

\section{Cell culture}

Under growth conditions $\mathrm{C} 2 \mathrm{C} 12$ cells were cultured in DMEM media with $10 \%$ FBS, when differentiating, media was switched to DMEM containing 2\%FBS. C3H 10T1/2 Cells were grown in Eagle's Basal medium with 2-mM L-glutamine, 1.5g/L sodium bicarbonate and Earle's BSS, with $10 \%$ FBS for growth conditions and 2\% FBS for differentiation conditions.

\section{siRNA transfection of cells}

Cells were transfected with Life Technologies Silencer Select siRNAs targeting MUNC, of target sequence ggaugagcugugugcuucu or cgaccaaugggagagagca, or commercial negative control silencer select siRNAs. siRNA was transfected at a final concentration of $30 \mathrm{nM}$ in growth medium, with $3 \mathrm{uL} / \mathrm{mL}$ total media volume of Lipofectamine RNAimax. siRNA and Lipofectamine was mixed in $2 \mathrm{~mL} / 10 \mathrm{~mL}$ total media volume Optimem for 25 minutes prior to addition to cells seeded onto plates at $25 \%$ confluency.

\section{RNA expression by qRT-PCR}

RNA was isolated by Trizol extraction, and cDNA synthesis was performed using Life Technologies Superscript III RT cDNA synthesis kits, using random hexamer priming. Prior to cDNA synthesis, RNA samples were treated with RQ1 RNAse free DNAse to eliminate potential DNA contamination of samples. qPCR was performed with BioRad iCycler, using iQ SYBR Green Supermix. Primers for the ncRNA screen were designed using BatchPrimer3.

\section{Northern Blotting}

RNA was isolated using Trizol extraction and run on $1 \%$ agarose gels containing $6.3 \%$ formaldehyde. RNA was transferred to Hybond $\mathrm{N}+$ membranes (GE Healthcare) and UV crosslinked. Membranes were hybridized to LNA probes against MUNC containing double DIG 
labels (Exiqon) in Ultrahyb buffer(Life Technologies). Membranes were washed in 2x SSC, and

0.2X SCC, and DIG signal was detected using DNA DIG labeling and detection kit (Roche). Chemiluminescent images were captured on a G:BOX geldoc system (Syngene).

\section{Microarray analysis}

RNA was hybridized to Affymetrix Mouse Exon ST arrays and analyzed for gene expression using Affymetrix Expression Console software and Microsoft Excel.

\section{Western Blotting}

Cells were lysed in IPH buffer and run on 10\% polyacrylamide SDS-PAGE gels and transferred to nitrocellulose membranes. Membranes were blocked for 30 minutes in 5\% Milk containing PBS-T, and incubated overnight with primary antibody in 3\% BSA. Secondary antibody incubation was carried out for 1 hour after washing, and at a 1:4000 dilution before washing and incubation with Millipore Immobilon HRP substrate. Chemiluminescent images were captured on a G:BOX geldoc system (Syngene). Antibodies and dilutions were used as follows, MyoD C20 1:250 (Santa Cruz), Myogenin 1:250 (Santa Cruz), MHC 1:250 (Millipore), Tubulin 1:3000(Sigma).

\section{ChIP and RIP studies}

Cells were washed in PBS and crosslinked with $1 \%$ formaldehyde for 10 minutes, and then treated with $0.15 \mathrm{M}$ glycine for 15 minutes. Cells were then PBS washed twice and lysed in lysis buffer containing $1 \%$ SDS with $50 \mathrm{mM}$ Tris $10 \mathrm{mM}$ EDTA, protease and RNAse inhibitors. Chromatin was sonicated to an average size of $300 \mathrm{bp}$, and incubated overnight with Protein G Dynabead/Antibody complexes, with 2 ug antibody per $1.5 \times 10^{\wedge} 6$ cells. After overnight incubation, Dynabeads were washed with RIPA containing $150 \mathrm{mM} \mathrm{NaCl}$ followed by $500 \mathrm{mM}$ $\mathrm{NaCl}$, RIPA containing $250 \mathrm{mM} \mathrm{LiCl}$, and twice with Tris-EDTA. Beads were then de- 
crosslinked overnight at $65^{\circ} \mathrm{C}$ and treated with Proteinase $\mathrm{K}$ (and RNAse A and $\mathrm{H}$ for $\mathrm{ChIP}$ ). DNA or RNA was then isolated by phenol:chloroform extraction or Trizol extraction respectively and analysed by qPCR or qRT-PCR.

\section{Chromatin Isolation by RNA purification}

CHIRP was performed as per (27), 15 oligonucleotide probes were designed with complementarity to MUNC, and 10 to LacZ using singlemoleculefish.com, pooled and labeled with biotin-16-UTP(Thermo-Fisher), using terminal deoxynucleotide transferase(NEB).

\section{Immunofluorescence}

Cells were plated on glass cover slips in the presence of 30nM siRNA. Cells were collected in growth medium or after 24,72 or 120 hours in differentiation medium. After 60 minutes, the cover slips were fixed with $4 \%$ formaldehyde in PBS, and permeabilized in $0.5 \%$ Triton X-100 in PBS. Coverslips were blocked in 5\% goat serum. Coverslips were incubated at room temperature with primary antibody for 1 hour, and Alexa 488 or 549 conjugated secondary antibody for 1 hour, with three TBS washes following each antibody incubation. Coverslips were then mounted with Vectashield mounting solution (Vector Laboratories). Antibodies used were anti-MyoD C-20 antibody (Santa Cruz Laboratories), and anti-Myosin Heavy Chain A4.1025 antibody (Millipore). Antibodies were diluted 1:200 in 5\% goat serum containing PBS.

\section{Microscopy}

Images were captured using a Nikon Microphot SA upright microscope equipped with a Nikon NFX35 camera using SPOT imaging software(Diagnostic Instruments Inc.) and a Nikon PlanApo 60x oil objective lens. Fluorescence images were acquired on the same day using the same exposure times, gamma, and gain between samples. Images were enhanced for brightness and contrast to the same extent within Photoshop. 


\section{Luciferase assays}

C3H10T1/2 Cells were transfected with pCMV-MyoD(addgene), pLPCX-MUNC, pRLTK, and pGL3-MyoDCER, or pGL3-MyoDDRR, pGL3-MCK enhancer, or pGL3-MEF2C RE. Luciferase activity was measured after 48 hours in $2 \%$ serum containing media using Dual Luciferase kit(Promega). 


\section{References:}

1. Wapinski O, Chang HY. Long noncoding RNAs and human disease. Trends Cell Biol. 2011 Jun;21(6):354-61.

2. Wang KC, Chang HY. Molecular Mechanisms of Long Noncoding RNAs. Molecular Cell. 2011;43(6):904-14.

3. Clark MB, Amaral PP, Schlesinger FJ, Dinger ME, Taft RJ, Rinn JL, et al. The reality of pervasive transcription. PLoS Biol. 2011 Jul;9(7):e1000625; discussion e1102.

4. Derrien T, Johnson R, Bussotti G, Tanzer A, Djebali S, Tilgner H, et al. The GENCODE v7 catalog of human long noncoding RNAs: analysis of their gene structure, evolution, and expression. Genome Res. 2012 Sep;22(9):1775-89.

5. Guttman M, Garber M, Levin JZ, Donaghey J, Robinson J, Adiconis X, et al. Ab initio reconstruction of cell type-specific transcriptomes in mouse reveals the conserved multi-exonic structure of lincRNAs. Nat Biotechnol. 2010 May;28(5):503-10.

6. Gomez JA, Wapinski OL, Yang YW, Bureau JF, Gopinath S, Monack DM, et al. The NeST long ncRNA controls microbial susceptibility and epigenetic activation of the interferongamma locus. Cell. 2013 Feb 14;152(4):743-54.

7. Lai F, Orom UA, Cesaroni M, Beringer M, Taatjes DJ, Blobel GA, et al. Activating RNAs associate with Mediator to enhance chromatin architecture and transcription. Nature. 2013 Feb 17.

8. Bernard D, Prasanth KV, Tripathi V, Colasse S, Nakamura T, Xuan Z, et al. A long nuclear-retained non-coding RNA regulates synaptogenesis by modulating gene expression. EMBO J. 2010 Sep 15;29(18):3082-93.

9. Gupta RA, Shah N, Wang KC, Kim J, Horlings HM, Wong DJ, et al. Long non-coding RNA HOTAIR reprograms chromatin state to promote cancer metastasis. Nature. 2010 Apr 15;464(7291):1071-6.

10. Maass PG, Rump A, Schulz H, Stricker S, Schulze L, Platzer K, et al. A misplaced lncRNA causes brachydactyly in humans. J Clin Invest. 2012 Nov 1;122(11):3990-4002.

11. Berkes CA, Tapscott SJ. MyoD and the transcriptional control of myogenesis. Semin Cell Dev Biol. 2005 Aug-Oct;16(4-5):585-95.

12. Yokoyama S, Asahara H. The myogenic transcriptional network. Cell Mol Life Sci. 2011 Jun;68(11):1843-9.

13. Gagan J, Dey BK, Dutta A. MicroRNAs regulate and provide robustness to the myogenic transcriptional network. Curr Opin Pharmacol. 2012 Jun;12(3):383-8.

14. Marks H, Chow JC, Denissov S, Francoijs KJ, Brockdorff N, Heard E, et al. Highresolution analysis of epigenetic changes associated with X inactivation. Genome Res. 2009 Aug;19(8):1361-73.

15. Plath K, Mlynarczyk-Evans S, Nusinow DA, Panning B. Xist RNA and the mechanism of X chromosome inactivation. Annu Rev Genet. 2002;36:233-78.

16. Orom UA, Derrien T, Beringer M, Gumireddy K, Gardini A, Bussotti G, et al. Long noncoding RNAs with enhancer-like function in human cells. Cell. 2010 Oct 1;143(1):46-58.

17. Asp P, Blum R, Vethantham V, Parisi F, Micsinai M, Cheng J, et al. Genome-wide remodeling of the epigenetic landscape during myogenic differentiation. Proc Natl Acad Sci U S A. 2011 May 31;108(22):E149-58.

18. Trapnell C, Williams BA, Pertea G, Mortazavi A, Kwan G, van Baren MJ, et al.

Transcript assembly and quantification by RNA-Seq reveals unannotated transcripts and isoform switching during cell differentiation. Nat Biotechnol. 2010 May;28(5):511-5.

19. Kong L, Zhang Y, Ye ZQ, Liu XQ, Zhao SQ, Wei L, et al. CPC: assess the proteincoding potential of transcripts using sequence features and support vector machine. Nucleic Acids Res. 2007 Jul;35(Web Server issue):W345-9. 
20. Chen JC, Ramachandran R, Goldhamer DJ. Essential and redundant functions of the MyoD distal regulatory region revealed by targeted mutagenesis. Dev Biol. 2002 May $1 ; 245(1): 213-23$.

21. Chen JC, Love CM, Goldhamer DJ. Two upstream enhancers collaborate to regulate the spatial patterning and timing of MyoD transcription during mouse development. Dev Dyn. 2001 Jul;221(3):274-88.

22. Rudnicki MA, Braun T, Hinuma S, Jaenisch R. Inactivation of MyoD in mice leads to up-regulation of the myogenic HLH gene Myf-5 and results in apparently normal muscle development. Cell. 1992 Oct 30;71(3):383-90.

23. Rudnicki MA, Schnegelsberg PN, Stead RH, Braun T, Arnold HH, Jaenisch R. MyoD or Myf-5 is required for the formation of skeletal muscle. Cell. 1993 Dec 31;75(7):1351-9.

24. Megeney LA, Kablar B, Garrett K, Anderson JE, Rudnicki MA. MyoD is required for myogenic stem cell function in adult skeletal muscle. Genes Dev. 1996 May 15;10(10):1173-83. 25. Chu C, Qu K, Zhong FL, Artandi SE, Chang HY. Genomic maps of long noncoding RNA occupancy reveal principles of RNA-chromatin interactions. Mol Cell. 2011 Nov 18;44(4):667-78.

26. Tapscott SJ, Lassar AB, Weintraub H. A novel myoblast enhancer element mediates MyoD transcription. Mol Cell Biol. 1992 Nov;12(11):4994-5003.

27. Wang Y, Yu Y, Tsuyada A, Ren X, Wu X, Stubblefield K, et al. Transforming growth factor-beta regulates the sphere-initiating stem cell-like feature in breast cancer through miRNA181 and ATM. Oncogene. 2011 Mar 24;30(12):1470-80. 


\section{Figures}

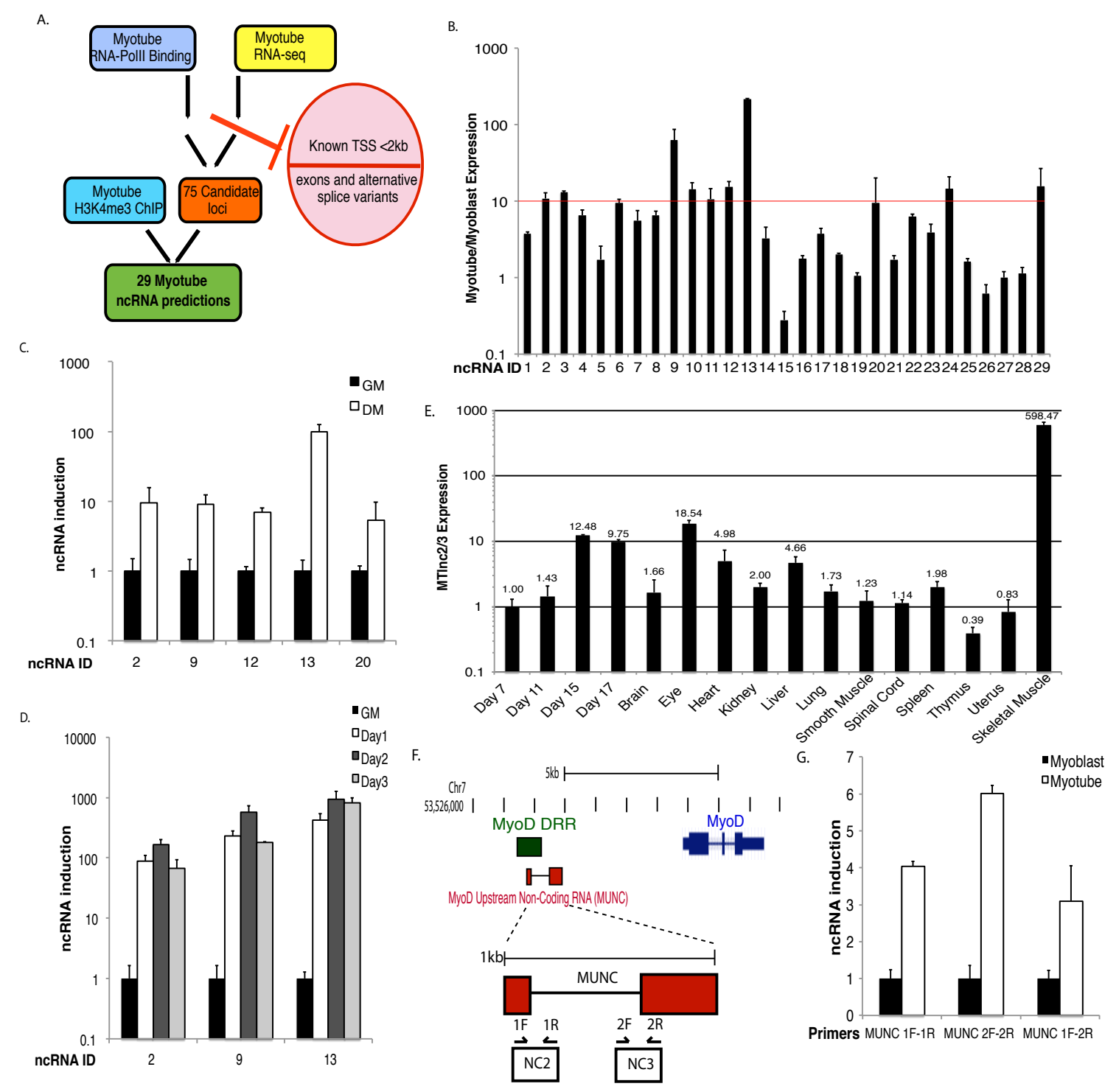

Figure 1. Predicted muscle specific non-coding RNAs are upregulated in myotubes.a, Schematic of in silico prediction of myogenically regulated long-non-coding RNAs. b, qPCR confirmation of predicted non-coding RNAs induced in myotubes versus myoblasts. 9 predicted RNA seq fragments at 7 independent genomic loci were found to be over 10-fold overexpressed in myotubes versus myoblasts.c. qPCR analysis of MyoD transfected transdifferentiating 10T1/2 fibroblasts for several predicted myogenically regulated lncRNAs when transferred to low serum differentiation media(DM) versus growth media(GM).d. RT-qPCR of lncRNAs from screen in differentiating $\mathrm{C} 2 \mathrm{C} 12$ Myoblasts on indicated days after switch to low serum differentiation media.e. RT-qPCR of indicated lncRNAs 2/3 (or MUNC) from mouse tissues showing high expression in skeletal muscle. f. Schematic of MUNC genomic region upstream of the MyoD1 locus, MUNC overlaps previously characterized Distal Regulatory Region (DRR) enhancer. g. RT-qPCR analysis of MUNC expression in primary myoblasts and myotubes. MUNC1F-1R and $2 \mathrm{~F}-2 \mathrm{R}$ primers measuring primary transcript, $1 \mathrm{~F}-2 \mathrm{R}$ primers measuring mature spliced ncRNA. Data represent mean $+/-\mathrm{SEM}, \mathrm{n}=3$. 

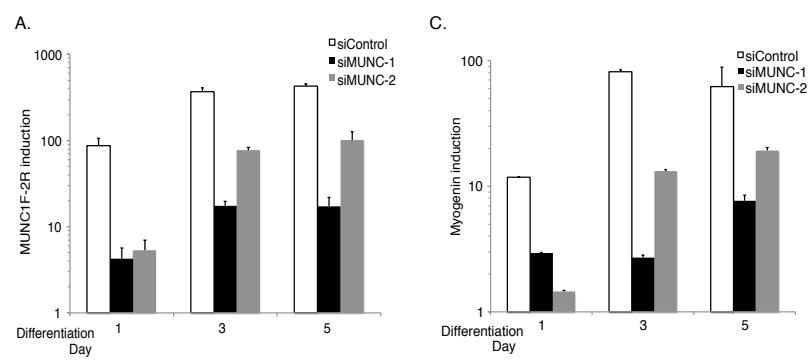

G.

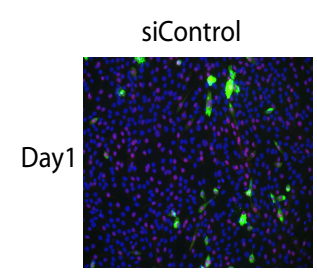

siMUNC-1

siMUNC-2
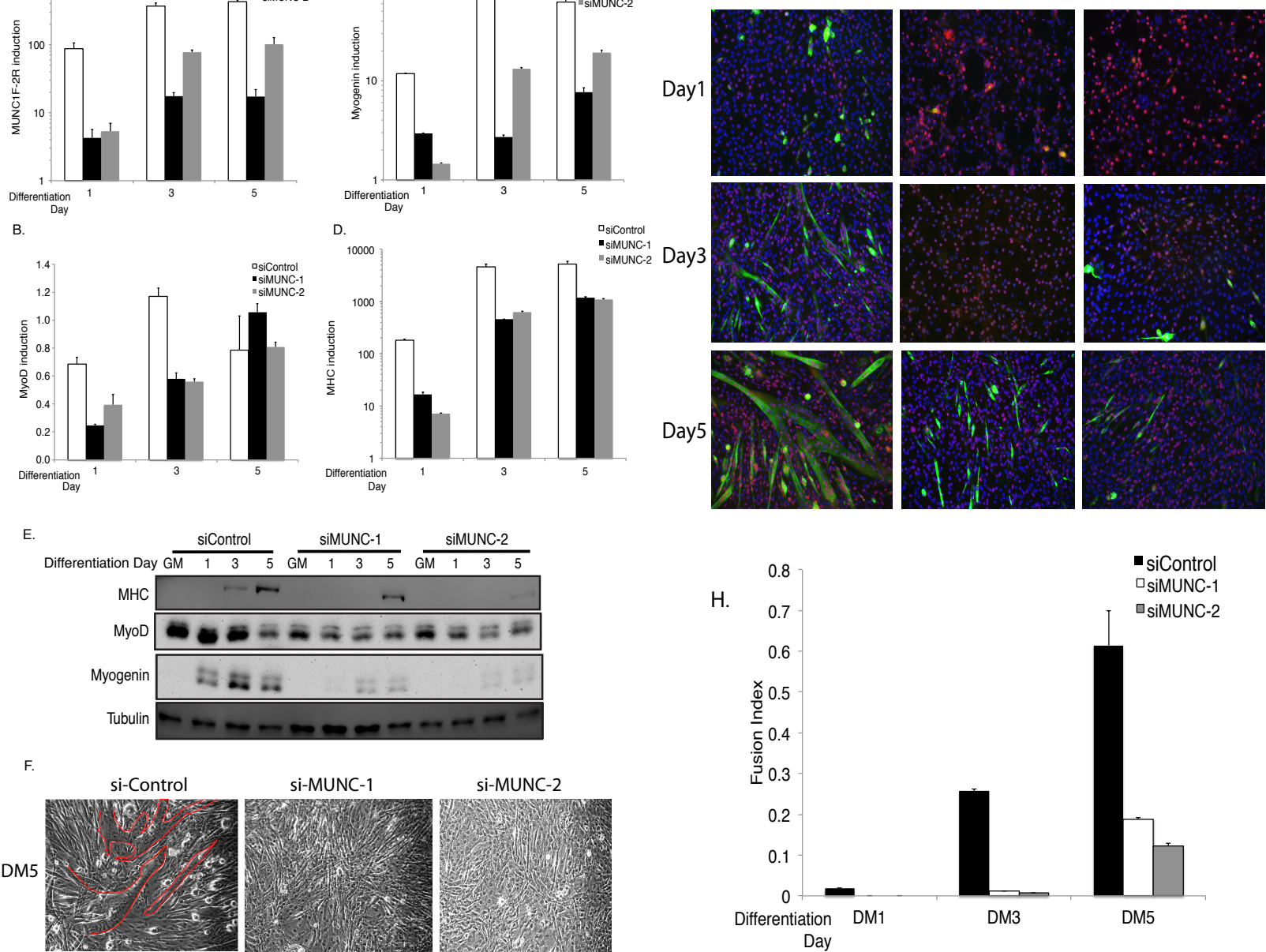

Figure 2. MUNC knockdown represses the induction of myogenic differentiation markers and impairs myotube formation in culture. a-d. RT-qPCR of MUNC and myogenic markers MyoD, Myogenin and Myh3 (MHC) during differentiation of C2C12 Cells incubated with either control siRNA or siRNA targeting the 5' or 3' end of MUNC. MUNC levels normalized to GAPDH. Data represent mean $+/-$ SEM, $n=3$ e. Western blot analysis of MHC, Myogenin and MyoD in differentiating $\mathrm{C} 2 \mathrm{C} 12$ cells. Independent siRNAs targeting MUNC reduce expression of these myogenic proteins. f. Bright field microscope image of differentiating $\mathrm{C} 2 \mathrm{C} 12$ cells incubated with control siRNA or siRNAs targeting MUNC. Myotube formation impaired with MUNC knockdown. Red lines enclose multinucleated myotube cells. g. Immunofluorescence of MHC (green) and MyoD (red) and DAPI (blue) in differentiating C2C12 cells. C2C12 incubated with control siRNA shows much greater formation of MHC positive, multinucleated cells on differentiation days 3 and 5 than cells incubated with siRNAs targeting MUNC. h. Fusion index of differentiating $\mathrm{C} 2 \mathrm{C} 12$ cells shows MUNC knockdown impairs myotube formation. Fusion index calculated by dividing number of nuclei contained within multinucleated cells by number of total nuclei in a field. Data represent mean $+/-$ SEM, $N=5$. 


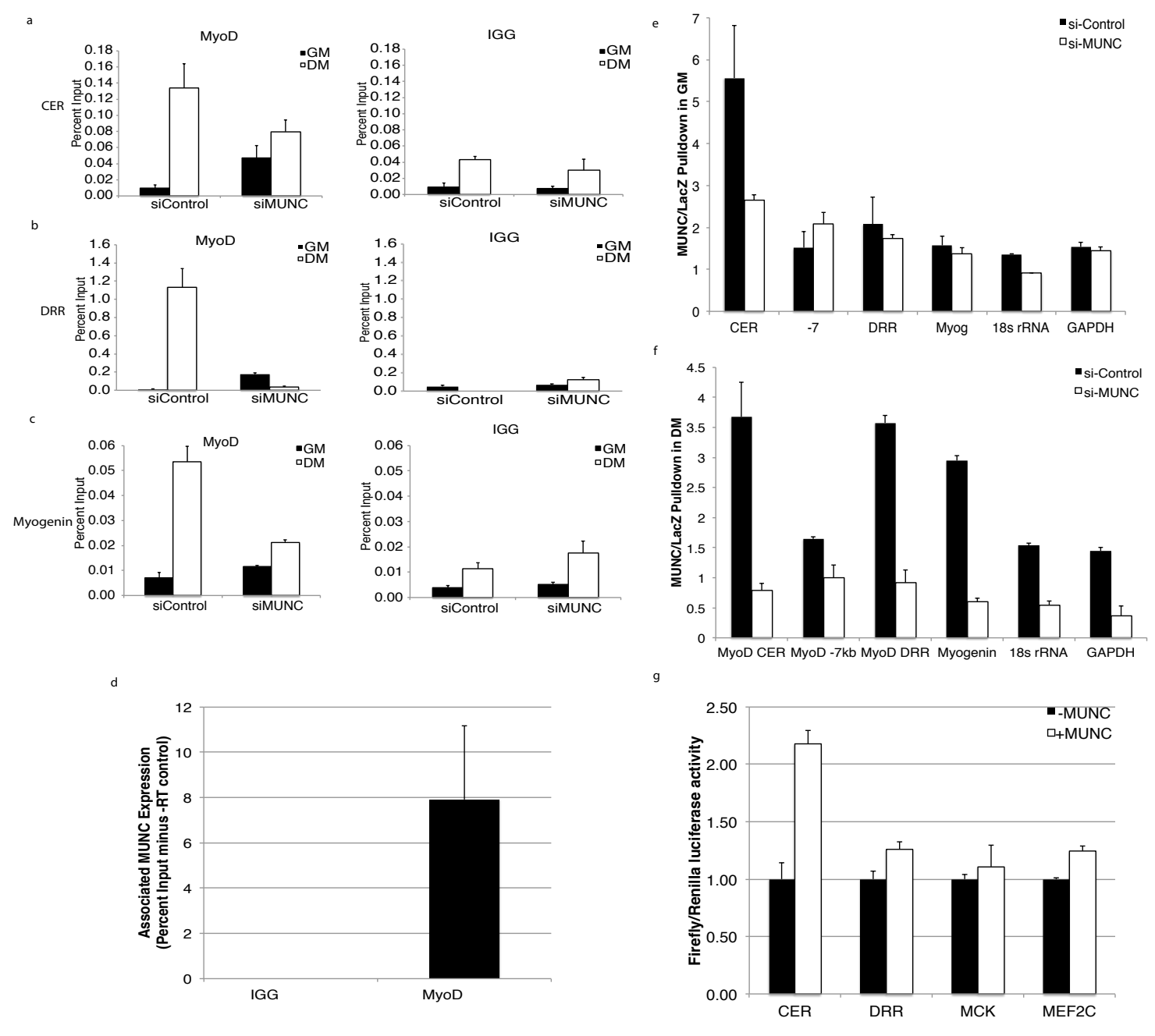

Figure 3. MUNC localizes to MyoD enhancer regions on chromatin and facilitates MyoD binding. a-c. MyoD ChIP at the MyoD Core Enhancer Region, Distal Regulatory Region, and Myogenin enhancer region. Control immunoglobulin G (IGG) ChIP to the same genomic sites. Cells were treated with either control siRNA or siRNA targeting MUNC and incubated in Growth or Differentiation Medium for 72 hours. Data represent mean +/- SEM, $n=3$. d. MUNC RNA precipitates with MyoD. RNA immunoprecipitation (RIP) of MyoD protein in C2C12 lysates. cDNA was prepared from RNA isolated by MyoD or IGG RIP, after 72 hours in differentiation media. Data represents MUNC expression as percent input, mean $+/-\mathrm{SEM}, \mathrm{N}=3$. e-f. MUNC ChIRP to several genomic loci. Cells were incubated with either control siRNA or siRNA targeting MUNC and examined in GM (e) or 72 hours of differentiation (f). MUNC RNA was captured by biotinylated oligonucleotides after crosslinking, and chromatin was isolated, and examined by qPCR for the MyoD enhancer regions, Myogenin enhancer region, and 18S and GAPDH repetitive loci. MUNC was localized at the CER in GM and DM, and was enriched in $\mathrm{DM}$ at the DRR and Myogenin enhancer regions. Data represent mean +/- SEM, $\mathrm{n}=3$. g. Luciferase activity for MyoD transcription activity during differentiation. C3H 10T1/2 cells were transfected with constructs expressing MyoD and MUNC, and pGL3 luciferase reporter constructs driven by the MyoD CER, DRR, MCK enhancer, or a MEF2C responsive element as well as pRL-TK control luciferase construct. Y axis : mean of Firefly/Renilla luciferase activity in +MUNC cells, normalized to - MUNC cells. Data represent mean +/- SEM n=3. 


\begin{tabular}{lrrlr}
\hline Chromosome & RNAseq start coordinate & RNAseq stop coordinate & RNAseqID & NCRNA_ID \\
\hline chr7 & 30766640 & 30766824 & MT_18580 & 1 \\
chr7 & 53626843 & 53627031 & MT_19022 & 2 \\
chr7 & 53627330 & 53627626 & MT_19023 & 3 \\
chr7 & 53629487 & 53629561 & MT_19024 & 4 \\
chr7 & 53629872 & 53629954 & MT_19025 & 5 \\
\hline chr14 & 22566952 & 22567040 & MT_141 & 6 \\
\hline chr14 & 32026105 & 32026292 & MT_248 & 7 \\
chr19 & 3765132 & 3765533 & MT_20418 & 8 \\
chr8 & 13201970 & 13202444 & MT_1230 & 9 \\
chr8 & 13202768 & 13202922 & MT_1231 & 10 \\
chr8 & 13203007 & 13203263 & MT_1232 & 11 \\
\hline chr8 & 126419999 & 126420073 & MT_2710 & 12 \\
chr1 & 20612312 & 20612490 & MT_2953 & 13 \\
chr6 & 29381128 & 29381504 & MT_24494 & 14 \\
chr6 & 88850233 & 88850446 & MT_25164 & 15 \\
\hline chr6 & 149263245 & 149263339 & MT_25901 & 16 \\
\hline chr11 & 46206645 & 46206735 & MT_21873 & 17 \\
\hline chr11 & 48687223 & 48687425 & MT_21890 & 18 \\
chr11 & 50026771 & 50027017 & MT_21919 & 19 \\
\hline chr11 & 58952620 & 58952746 & MT_22298 & 20 \\
chr16 & 23989822 & 23989993 & MT_6896 & 21 \\
\hline chr3 & 14530455 & 14530643 & MT_8317 & 22 \\
chr15 & 27958322 & 27958509 & MT_11215 & 23 \\
\hline chr4 & 119962964 & 119963170 & MT_26893 & 24 \\
chr2 & 91789193 & 91789586 & MT_13961 & 25 \\
\hline chr9 & 21891831 & 21892015 & MT_28975 & 26 \\
\hline chr13 & 75846117 & 75846436 & MT_16126 & 27 \\
\hline chr13 & 75846651 & 75847469 & MT_16127 & 28 \\
chr5 & 31912782 & & & 29 \\
\hline & & & \\
\hline
\end{tabular}

Supplemental Table 1. In silico predicted putative myogenically regulated long noncoding RNAs. Genomic locations and identifiers of transcripts differentiatlly expressed in myotubes over $200 \mathrm{nt}$ in length which overlap with myotube specific H3K4me3 and RNA PolII occupancy located outside of known protein coding loci. 29 of these putative lncRNAs were identified in silico for experimental confirmation. 


\begin{tabular}{|l|l|l|l|}
\hline $\begin{array}{l}\text { NCRNA_I } \\
\text { D }\end{array}$ & $\begin{array}{l}\text { RNASEQ_I } \\
\text { D }\end{array}$ & Adjacent Gene & Description \\
\hline $2-3$ & $19022 / 3$ & MyoD & MyoD \\
\hline $9-10$ & $1230 / 1$ & Grtp1 & Novel GH responsive gene \\
\hline 12 & 2710 & Nup133 & Nuclear Pore protein \\
\hline 13 & 2953 & $\begin{array}{l}\text { Pkhd1, miR- } \\
206 / 133 b\end{array}$ & $\begin{array}{l}\text { Polycystic kidney and hepatic disease 1, } \\
\text { miR-206/133b }\end{array}$ \\
\hline 20 & 22298 & Obscn & Obscurin \\
\hline 24 & 26893 & Scmh1 & Sex comb on midleg homolog protein \\
\hline 29 & 30734 & Mrp133 & $\begin{array}{l}\text { mitochondrial ribosomal protein L33 } \\
\text { (Mrpl33) }\end{array}$ \\
\hline
\end{tabular}

Supplemental Table 2. Experimentally confirmed putative myogenically regulated long noncoding RNA. Identifiers, fold change and neighboring genes of predicted lncRNAs upregulated in myotubes versus myoblasts, confirmed by qRT-PCR. Each fragment was upregulated 10 fold in myotubes or greater. 

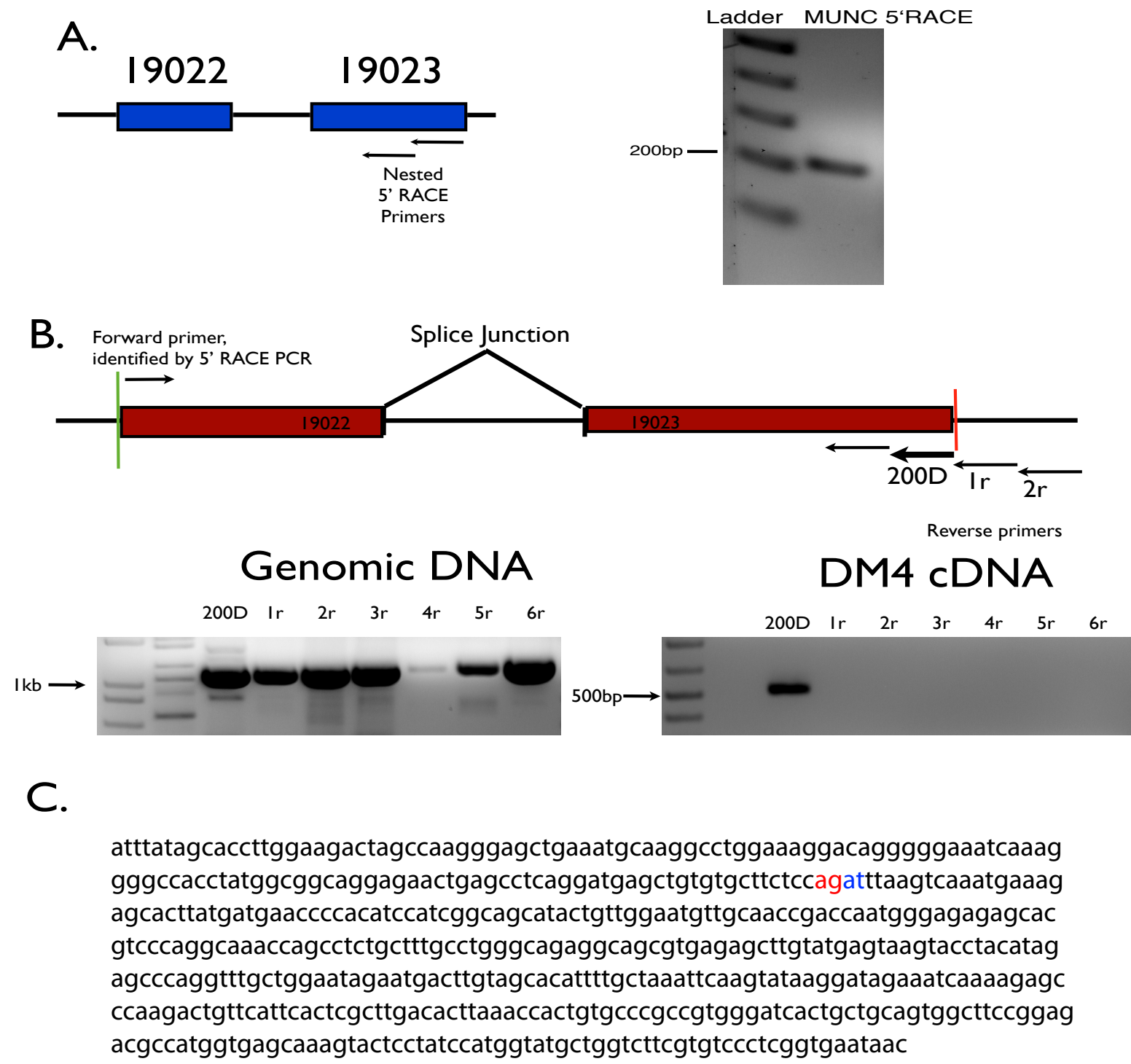

Supplemental Figure 1. Characterization of MyoD Upstream NonCoding transcript. a, Schematic of and PCR product generated from 5' RACE PCR characterizing the 5' end of MUNC using cap capture method. b, PCR primer walking to determine the 3' end of spliced MUNC transcript. c, Sequence of spliced MUNC, red represents 5' splice site, and blue represents 3' splice site. 
A.

\begin{tabular}{|c|l|l|l|}
\hline \multirow{3}{*}{ HOMOLOGY FEATURES } & HIT NUM & 0 & \\
\cline { 2 - 4 } & HIT SCORE & 0.0 & \\
\cline { 2 - 4 } & FRAME SCORE & 0.0 & \\
\hline \multirow{3}{*}{ ORF_FRAMEFINDER } & COVERAGE & $23.17 \%$ & \\
\cline { 2 - 4 } & LOG-ODDS SCORE & 25.83 & \\
\cline { 2 - 4 } & TYPE & Partial & \\
\cline { 2 - 5 } & & \multicolumn{2}{|l|}{} \\
\hline
\end{tabular}

legend: non-coding

B.

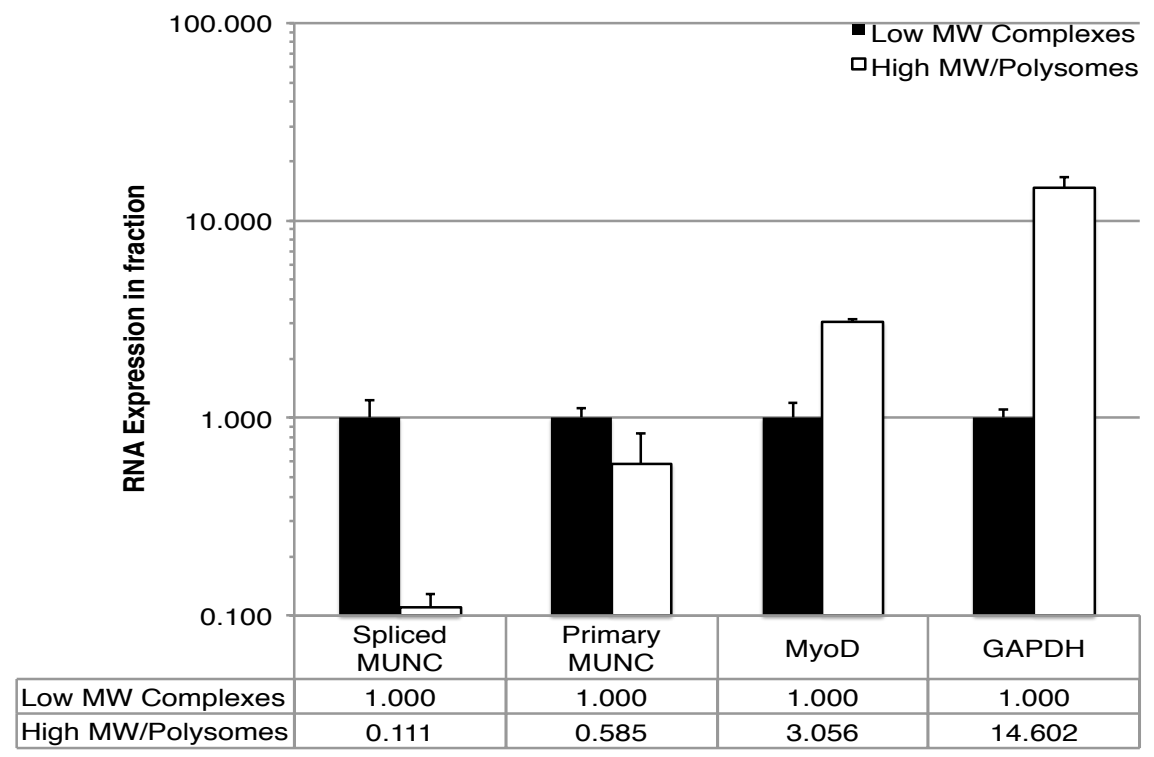

Supplemental Figure 2. Putative IncRNA MUNC predicted to have low coding potential and is not associated with polysomes during differentiation. A. Analysis obtained from the Coding Potential Calculator indicating based on homology and ORF attributes MUNC is likely a non-coding transcript. B. qRT-PCR of Polysome fractionation of differentiating C2C12 myotubes shows MUNC primarily associated with low molecular weight/monosome fraction, as opposed to the mRNA transcripts for MyoD and GAPDH which are associated with the actively transcribing polyribosome fraction. Data represents mean $+/-\mathrm{SEM}, \mathrm{n}=3$. 

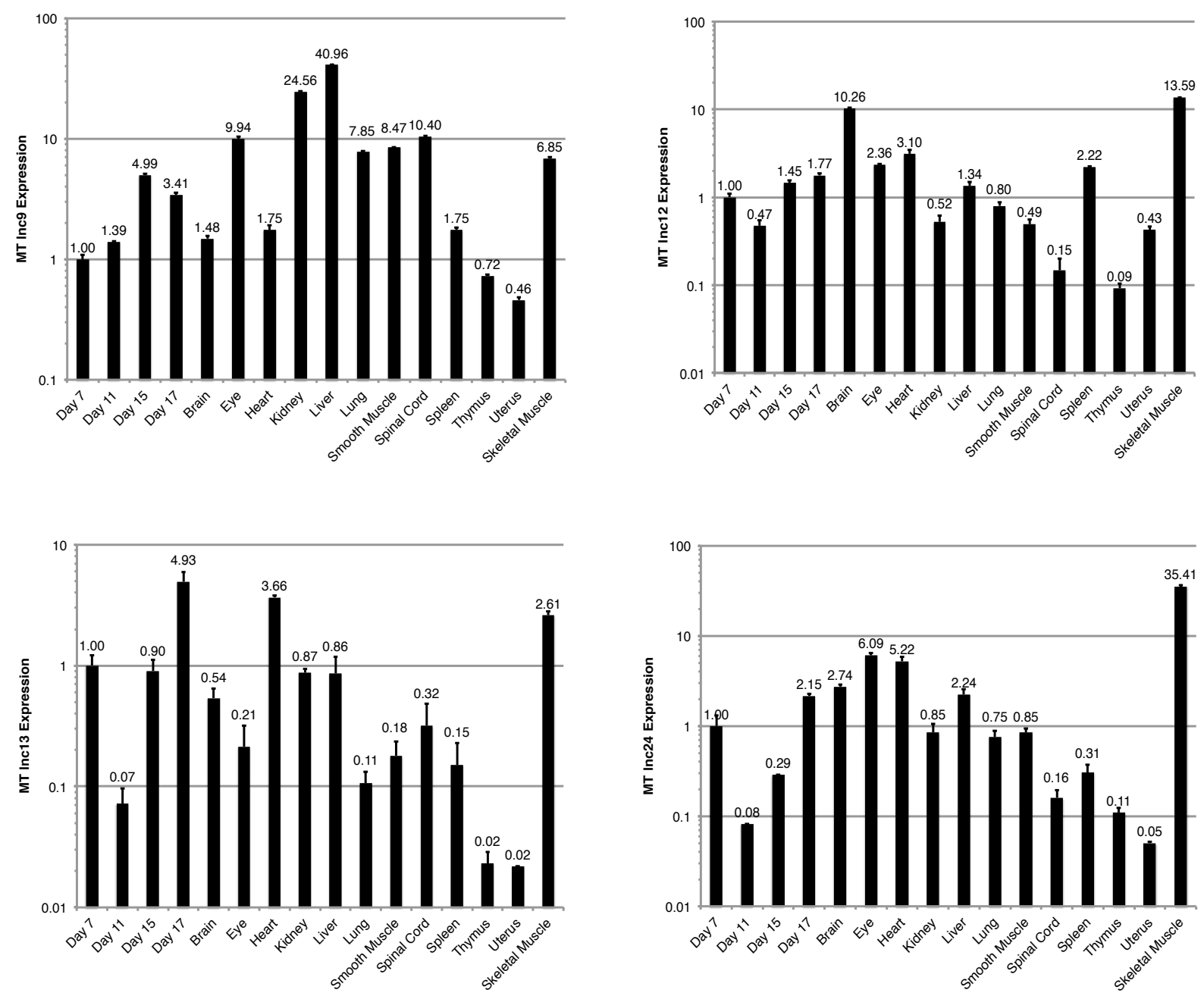

Supplemental Figure 3. Tissue expression of experimentally confirmed myogenically upregulated putative long noncoding RNA. a-d. qRT-PCR expression values of indicated transcripts across a panel of embryonic and adult mouse tissue samples. Values are normalized to expression of RPS13, a housekeeping gene with low tissue variability and plotted relative to expression in Day 7 embryonic tissue. Data represents mean $+/-$ SEM, $n=3$. 


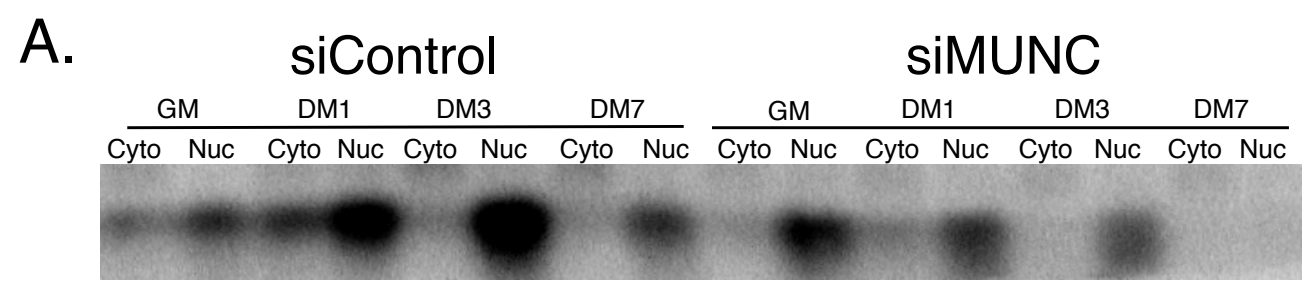

\section{Supplemental Figure 4. Northern Blot for MUNC expression in differentiating C2C12 myoblasts.}

a. RNA from $\mathrm{C} 2 \mathrm{C} 12$ myoblasts incubated with control siRNA or siRNA targeting MUNC and placed under differentiation conditions for increasing duration was obtained and run on a denaturing agarose gel and transferred to positively charged nylon membranes. The membranes were hybridized with locked nucleic acid oligos complementary to MUNC, which were labeled with dual digoxygenin tags. MUNC expression increases, and becomes more nuclear during differentiation, this increase is blocked by siRNA treatment targeting MUNC. 


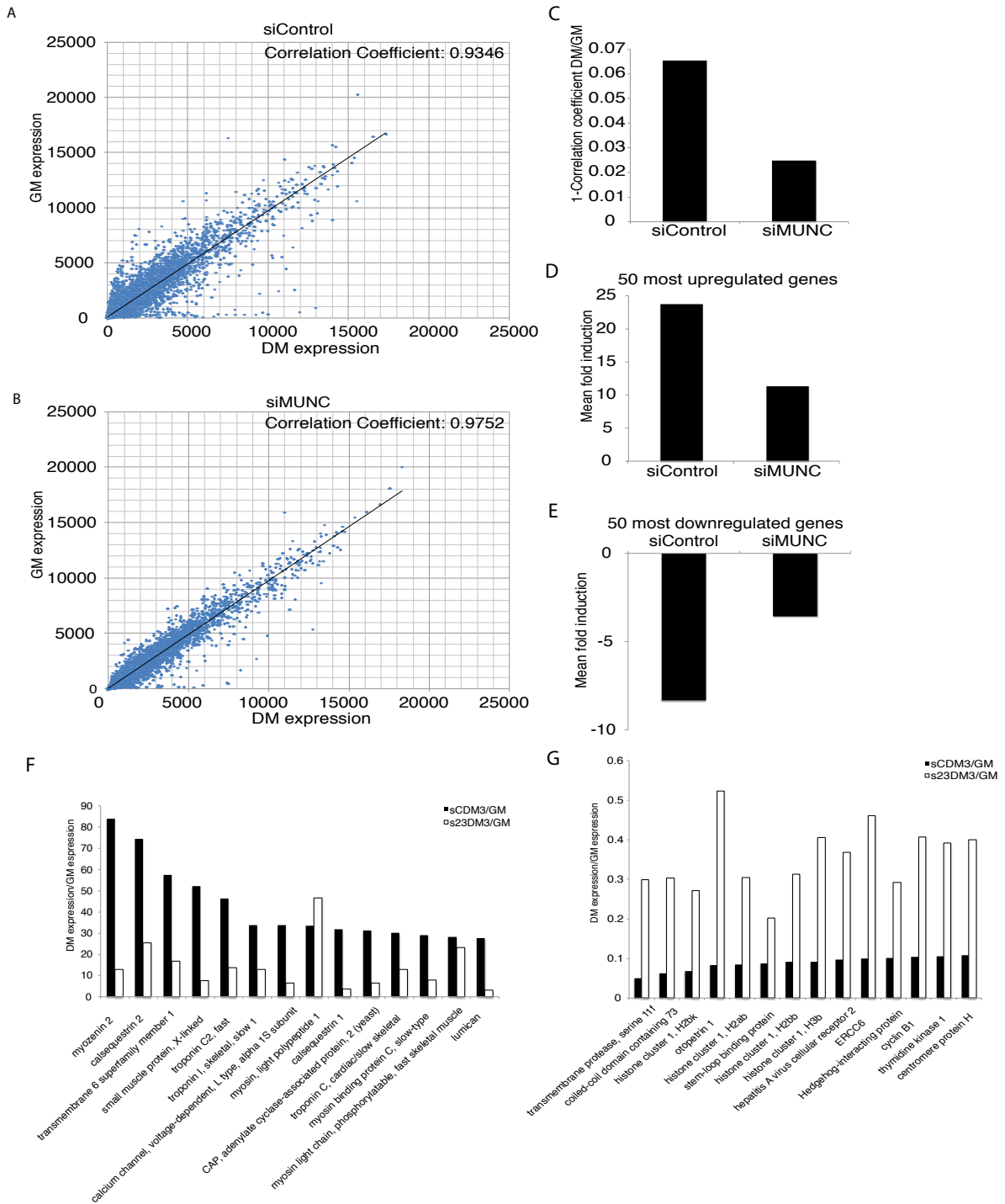

\section{Supplemental Figure 5. Global gene expression changes that occur during skeletal} myogenesis are inhibited by MUNC depletion. a-b Scatter plot of gene expression in growth medium(GM) versus 72 hours in differentiation medium(DM3) with either control siRNA (a) or MUNC siRNA (b). c. 1 minus correlation coefficient of gene expression profiles of GM versus DM3 cells with either siControl or siMUNC. 1 minus correlation coefficient greater in control population, indicating myogenic gene expression changes require presence of MUNC. d-e. Mean expression fold change of the 50 most upregulated (d) or downregulated (e) genes in control differentiating cells. This fold change is suppressed in siMUNC cells. f-g. Expression fold change of the 14 most upregulated (d) and downregulated (e) genes in control cells versus those in siMUNC cells. In all but 1 cases, this change in gene expression is greatly suppressed by MUNC knockdown. 


\section{Chapter 4}

\section{Summary and Future Directions:}

\section{A. microRNA mediated regulation of the DNA damage response and the implications of such regulation for cancer. \\ i. microRNA expression can mediate radiation sensitivity in cancer and microRNA expression changes that occur following exposure to radiation can affect cells' ability to repair DNA damage.}

We originally set out to examine microRNA expression in response to DNA damage in breast cancer cells, performing our screening in two cell lines, MCF7 as SK-BR-3. Using locked nucleic acid microarrays specific to the currently known set of human microRNAs, we examined microRNA expression 24 hours following treatment with ionizing radiation to identify those whose expression were highly up or downregulated as consequence of the DNA damage response. This original screen however yielded surprisingly few microRNAs which were regulated in common between these two cell lines, and indeed, no microRNAs were upregulated more than two fold, and those which had high fold inductions were mostly due to low expression levels which were near the threshold of detection. This may have been partly because we examined expression at a relatively late time point following DNA damage, but we were interested in microRNAs which could robustly affect a phenotype in treated cells, and believed any transient expression changes that lasted less than 24 hours to have a limited usefulness in conferring a long term phenotype upon cells. The most likely explanation though is while there are many examples where microRNA expression profiles dramatically change and affect cellular phenotypes during development, greatly influencing commitment to different tissue lineages and terminal differentiation, fewer examples have been found where microRNA expression changes 
dramatically in response to a transient stimulus. Since initiating this study, other groups have also found microRNAs whose expression changes in response to DNA damage, but these changes are often much smaller than those seen in development, often specific to the tissue or cell line being examined, and often in contradictory directions in different studies. This variability can be partly attributed to variations in the time elapsed after inflicting DNA damage, cell type, or damage modality (1).

\section{ii. MicroRNAs whose expression correlates with cells' DNA damage sensitivity and are upregulated following irradiation}

In order to exploit the ability of microRNAs to confer cellular phenotypes, and tease out more subtle transient changes in expression in response to DNA damage that was still biologically significant, we decided to examine which microRNAs had differential expression between two cell lines with differential radiosensitivity, and intersect those with microRNAs upregulated following radiation in our more radiosensitive cell line. We found MCF7 cells to have much greater sensitivity to ionizing radiation treatment than SK-BR-3 cells, so we adjusted our analysis to find microRNAs whose expression was higher in MCF7, and which were upregulated following DNA damage. This analysis proved more fruitful as it yielded a number of microRNAs that were only subtly upregulated following irradiation but were previously found to have interactions with the DNA damage response. miR-34a was one of these microRNAs, and had previously been known to play a role in the p53 dependent response to DNA damage, itself facilitating the tumor suppressive function of p53(2). miR-21, another microRNA found in this analysis, is also upregulated after DNA damage, while having anti-apoptotic, oncogenic roles(3, 4). Upregulation of two prominent microRNAs of opposing function within a single cell type suggests that there may exist opposing mechanisms of microRNA regulation in cells that keep each other in check, and determination of cell phenotype may depend on which direction this balance is tilted. 


\section{iii. Regulation of miR-99 family in response to radiation and hormonal stimulus}

Of prime interest was the finding that expression of the tumor suppressive miR-99 family of microRNAs and its upregulation after DNA damage correlated with radiation sensitivity. Previous work from our lab had identified the miR-99 family as being suppressed in more advanced androgen independent prostate cancer, and this screen also found its expression decreased in the more aggressive breast cancer line we examined. Indeed, as well as in the hormone responsive MCF7 cells, we also found miR-99 to be upregulated in androgen dependent LnCAP cells suggesting a potential hormone responsive element in its regulation. The finding that expression of exogenous miR-99 could sensitize the hormone independent cell lines to radiation (is that what you meant?) suggests that decrease of miR-99 may be a common mechanism by which cancers may evade the apoptotic response and become resistant to therapy. Further study is necessary to determine the regulation of this family of microRNAs, and how this regulation is altered during cancer progression. In this vein our lab recently discovered the miR99a/let7c/miR-125b-2 cluster is epigenetically suppressed by androgen treatment(5). Loss of the requirement for androgen to maintain this silencing is likely a mechanism that contributes to the more aggressive phenotype of androgen independent cancers. It will be of interest to see if this silencing of miR-99 also occurs in response to estrogen signaling in ER-positive breast cancers, and whether miR-99 becomes more permanently decreased when breast cancers escape hormone dependence.

\section{iv. microRNAs that target chromatin remodelers and their significance in cancer and therapeutic resistance}

The upregulation of miR-99 following DNA damage in the more radiosensitive cell lines presents an interesting hypothesis: that more differentiated cells can prime themselves to enter apoptosis when confronted with multiple mutagenic insults in a short duration of time. Loss of 
this mechanism might make them more likely to accrue oncogenic mutations if repair and recovery continues to occur in response to repeated DNA damage, potentially resulting in genomic instability. Oddly, rather than targeting specific components of the more error prone Non-Homologous End Joining (NHEJ) pathway of repair, or targeting anti-apoptotic proteins, miR-99 mediates survival following DNA damage by targeting the chromatin remodeling factor SNF2H. SNF2H is required for both NHEJ(6) as well as Homologous Recombination (HR) (7) DNA repair. The loss of miR-99 repression of SNF2H makes cells more efficient at repairing DNA breaks and evading apoptosis. SNF2H is also important for S-phase progression, in all making it a good target for derepression during cancer progression. Interesting, it was recently found that overexpression of the RSF1 complex, which SNF2H is a member of, along with Cyclin E1 in a p53 mutant background was sufficient to initiate tumorigenesis, confirming upregulation of certain chromatin remodelers can be powerful drivers of transformation(8).

There have been numerous instances of chromatin remodeling factors contributing to the regulation of microRNAs involved in the progression of cancer(8-12). Conversely, there have been a number of chromatin remodelers targeted by microRNAs, contributing to cancer phenotypes(13-16). Interestingly miR-21, one of the microRNAs upregulated in our screen in response to DNA damage, targets the chromatin remodeling factors ANP32A and SMARCA4, indicating multiple microRNAs targeting multiple similar pathway components may function in parallel in response to stimulus(16). Similarly, parallel up-regulation of multiple members of the miR-99 family following irradiation, which all can target SMARCA5/SNF2H, indicate the importance of these microRNAs, and the regulation of chromatin remodeling in a cell's response to DNA damage. These examples suggest that the interplay of microRNAs and chromatin remodeling proteins is an important factor in cancer development, and these regulatory loops should be further examined to gain insight into the genetics of particular cancers, as well as to develop new therapeutic strategies. 


\section{v. Higher order cellular microRNA response}

While many studies have identified microRNAs whose expression is regulated following exposure to DNA damaging agents, beyond a few specific cases, there is much variability in the regulation of an individual microRNA depending on tissue and cell type as well as the time point examined and DNA damaging agent utilized. In order to identify more general trends of the microRNA response to DNA damage, it may be useful to employ systems level analysis of the microRNA expression profile focusing on common targets of those microRNAs whose expression changes. Prediction of microRNA targets remains difficult, as the algorithms used are riddled with false positive predictions, and it will be useful to intersect mRNA expression changes (post-irradiation) with microRNA expression changes (also post-irradiation) and target prediction to find bona fide mRNA targets that can then be confirmed by more targeted experiments. The recent study from our lab identified targets of the miR-99a/let-7c/125b cluster by this type of analysis and was able to confirm a number of bona fide mRNA targets regulated by this cluster with significantly more accuracy than bioinformatic prediction alone(5). Unfortunately this approach will only identify mRNAs that are degraded by the action of microRNAs, which will miss a significant population where only the translation is inhibited without any change in the mRNA levels. With improved bioinformatic accuracy, it may be possible to integrate targets of multiple radiation-regulated microRNAs to identify key pathways that are activated/repressed by microRNA changes following DNA damage. These changes in the pathway level may be missed when we focus only on the subtle changes in individual microRNAs and their target mRNAs. Additionally, large scale screens with luciferase reporters containing microRNA target sites can be carried out to identify microRNAs that are sufficiently altered following radiation to cause changes in the reporter expression. Such a global screen should identify biologically significant microRNA expression changes that actually affect their target expression following radiation. Hopefully these more advanced types of analysis should 
identify more global trends in microRNA expression changes and the resulting cellular phenotypes due to changes in target expression, and clarify much of the contradictory evidence currently on record.

\section{vi. Molecular mechanisms of the efficacy of fractionated radiotherapy}

Of additional interest is the question of whether and what molecular mechanisms contribute to the efficacy of fractionated radiotherapy and how to exploit these mechanisms to increase its success at specifically killing cancerous cells. The use of multiple smaller doses of radiation when treating cancer is typically thought to allow repair of sublethal DNA damage while killing rapidly dividing cancerous cells, to prevent the significant damage to normal tissue and associated morbidity that can occur with large single doses of radiation(17). However our study suggests that in less transformed, more radiosensitive dividing cells, like less aggressive cancers, microRNAs like miR-99 may be induced by one fraction of radiation to decrease the efficacy of DNA repair after subsequent fractions of radiation and thus contribute to the success of fractionated therapy. Additionally, loss of this microRNA induction and accompanying sensitization to the second fraction of radiation appears to be common in the two more radioresistant, hormone independent cancers we examined. Thus, these latter cancers are expected to be less sensitive to fractionated therapy, at least through the mediation of miR-99. Not only does this suggest that low miR-99 will be a prognostic marker for radiation resistance of cells, but also suggests a potential therapeutic approach. If these microRNAs can be reintroduced locally to cancers, they could re-sensitize cancers to DNA damage. Additionally, a finer definition of the time when cells upregulate and maintain expression of microRNAs suppressing DNA repair will help guide new protocols for fractionated radiation treatment. This more rational approach is expected to be more successful than the current empirical approach where the dose and time of delivery of fractions is arbitrarily designed without taking into 
account temporal variation of the gene and microRNA expression profiles in the cancers following the each fraction.

In sum it has become clear that microRNAs are a significant player in cancer development and progression, and will be an important future consideration for molecular staging, prognostication, and therapeutic targeting of cancers. MicroRNAs have been found which mediate epithelial to mesenchymal transition (EMT) in metastatic cancer, oncogenic signaling, and now radiation resistance. Further improvements in basic research concerning microRNAs can come in the form of increasing the accuracy of mRNA target prediction algorithms, which still generate considerable false positives. However, as our understanding of the mechanism of microRNA action has matured in the last decade, great advances can be expected in the translational applications of microRNAs for improving cancer therapy. Correlating large scale microRNA expression profiling of cancers with treatment efficacy should hopefully allow tailoring of advanced treatment regimens to smaller subgroups of cancers, and increasing the likelihood of therapeutic success. Additionally, advances in using microRNAs as drugs hold much promise in specifically targeting cancers, decreasing reliance on traditional cytotoxic therapies.

\section{B. The role of long noncoding RNAs in skeletal myogenesis}

Recent developments in the rapidly expanding field of long noncoding RNAs have raised many new questions about gene regulation in higher eukaryotes. While long noncoding RNAs have been found to direct gene silencing, regulate splicing, facilitate enhancer activity, and regulate microRNA targeting, we still know little of the mechanisms by which they act in these roles, the significance of their relative lack of evolutionary conservation, and how to predict their functionality based on their sequence. We have identified a set of long noncoding RNAs that are upregulated during skeletal myogenesis, and characterized a novel long noncoding RNA 
upstream of MyoD in the genome, which we have named MUNC, whose expression is necessary for differentiation of mouse myoblasts into skeletal myotubes.

\section{i. Further mechanistic characterization of MUNC}

Expression of MUNC is required for binding of MyoD at a number of promoters, including its own regulatory regions and that of myogenin. Preliminary data suggests that MUNC binds to MyoD, and is also associated with some MyoD responsive promoter regions, indicating it may be directly facilitating MyoD binding to DNA in trans. This needs to be experimentally confirmed by in vitro binding assays, as well as in vivo rescue experiments to show that the deficiency in myogenesis that occurs upon depletion of MUNC can be abrogated by expression of exogenous MUNC. MUNC could be simply an enhancer-RNA (eRNA) that promotes the expression of $M y o D$ in cis similar to other enhancer-like lncRNAs, which facilitate the looping of chromatin and recruitment of transcriptional proteins in cis at enhancers (Orom et al., 2010a; Orom et al., 2010b; Lai et al., 2013). However, the two-fold defect in MyoD expression is much less than the ten-fold effect of Myogenin or MHC expression in myoblasts induced to differentiate after depletion of MUNC. In addition, the localization of MUNC to the myogenin enhancer during differentiation, and the stimulation of exogenous MyoD's ability to activate a MyoD-responsive promoter suggest that MUNC has trans functions independent of being a cis e-RNA required for the full activity of the MyoD promoter. A prediction of this hypothesis is that expression of exogenous MyoD alone will not be sufficient to rescue the defects in myogenesis seen with MUNC depletion. Establishing MUNC as a cis e-RNA will still likely be of great interest as another important example of the class of lncRNAs, but our preliminary results suggest that MUNC acts by an independent mechanism with a trans component, which suggests a new, as yet unidentified class of lncRNA. 
Additionally, we have observed that MUNC appears to exist in multiple isoforms, with both a longer unspliced isoform as well as a shorter spliced isoform, both being upregulated during myogenesis. Characterization of which (if not both) of these isoforms is functional, and whether splicing of the long isoform is required for proper processing and localization of the spliced isoform are questions which will need to be addressed. The ability of MUNC to stimulate MyoD action on a luciferase reporter in 10T1/2 cells (Chapter 3, Figure 3g.) provides a simple assay to characterize the functional domains of MUNC by targeted mutations in MUNC. The same mutations of MUNC can be utilized in parallel to examine whether its ability to stimulate transcription from MyoD dependent enhancers is critical for its ability to stimulate myogenesis. Also, while we have identified some genomic target sites and protein partners of MUNC through a candidate approach, we should also globally screen for genomic loci as well as binding partners that can interact with MUNC through pull-down of MUNC RNA and sequencing associated DNA (ChIP-seq), as well as mass spectrometry of associate proteins. The structure-function characterization of MUNC, described above with MyoD-responsive luciferase reporters, can be further improved with the identification of new protein and genomic binding partners of MUNC, which can be functionally analyzed through similar luciferase assays.

\section{ii. Does MUNC belong to a new class of trans acting IncRNAs?}

If it can be confirmed that MUNC is acting in trans to bind to and facilitate the function of MyoD, further questions arise regarding whether more such lncRNAs of similar mechanism exist in the genome, and what their contributions are to the development of other tissue lineages. If MUNC is acting in trans, this suggests its location in proximity to MyoD in the genome may be more related to their co-evolution, rather than its activity as a cis element in the $M y o D$ promoter. This would encourage screens for lncRNAs encoded near transcription factors known to function in differentiation and share similar expression profiles. Such screens may identify more examples of this class of lncRNA-transcription factor pairing, and open new avenues of 
study for regulation of tissue development. Prediction of functional conservation of ncRNAs remains difficult, and there have been examples of poorly conserved ncRNA sequences that have conserved functionality and genomic locus between species. Until prediction of ncRNA secondary structure and function based on sequence becomes more effective, it is impossible to predict the consequences of mutations/sequence polymorphism in these loci and whether homologs have preserved function due to compensatory mutations. Thus it may be necessary to rely on other methods, like preservation of the expression profile or genomic location of a given lncRNA as well as secondary structural motifs in the lncRNA, rather than sequence conservation, to identify lncRNAs with preserved functions between species.

\section{iii. Characterization of other IncRNAs upregulated during myogenesis}

Of additional interest will be the characterization of the functions of the other lncRNAs identified in our screen. Several other putative lncRNAs were upregulated during myogenesis, and we have yet to test their requirement for efficient myogenesis. Of particular interest is the lncRNA, MT-2953, which was upregulated several hundred fold in our initial screen. The target gene most proximal to MT-2953 is PKHD1, which is a receptor protein whose mutation results in autosomal recessive polycystic kidney disease, and does not immediately appear to be a likely candidate gene involved in skeletal muscle development. However, fascinatingly, the gene located on the other side of MT-2953, though approximately 50 kilobases away on the chromosome, is that encoding the miR-206/133b cluster, both important microRNAs induced during and involved in skeletal myogenesis. The significance of MT-2953 upregulation during myogenesis and whether MT-2953 can regulate miR-206/133b expression, or plays some role in the action of these microRNAs warrants further study. This also brings up an interesting question regarding whether microRNAs actively regulate functional long non-coding RNAs in cells. There have been examples where lncRNAs and the 3' UTRs of coding genes or pseudogenes that "sponge" away microRNAs, allowing expression of other target genes. There has as yet been 
enough study of microRNAs that actively target lncRNA expression to confer a cellular phenotype through lncRNA downregulation, likely due to the dearth of well characterized lncRNAs. Considering the importance of microRNAs for tissue development, and the rapidly emerging plethora of roles for lncRNAs, it is likely these types of microRNA-lncRNA interplay will present interesting new directions for RNA research.

The rapidly growing field of lncRNA mediated regulation of gene expression and cellular function represents the next frontier of research in molecular biology. Higher eukaryotes are proving to have layers upon layers of regulation dictating gene expression between tissues and cell types that utilize mechanisms including DNA methylation, histone modification, chromatin remodeling, transcription factor recruitment to DNA, co-regulation of transcription factors by other protein cofactors, alternative splicing, posttranscriptional regulation of mRNA stability and regulation of translational efficiency, not to mention posttranslational protein modification and turnover mechanisms. It is becoming clear in higher eukaryotes that many if not all of these processes may also be facilitated and regulated by noncoding RNAs that run a wide gamut of sizes, expression patterns and mechanisms of action. While we now understand some of these mechanisms, particularly in the area of short RNAs typified by microRNAs, the rules of evolution and conservation of long noncoding RNAs, as well as how to predict their functions and interactions are yet poorly understood. Study of this rapidly growing class of molecules should hopefully lead to a much better understanding of the complexity seen in higher eukaryotes, as well as provide targets for characterizing and treating the complex diseases seen in humans. 


\section{References:}

1. Wang Y, Taniguchi T. MicroRNAs and DNA damage response: implications for cancer therapy. Cell Cycle. 2013 Jan 1;12(1):32-42.

2. Chen F, Hu SJ. Effect of microRNA-34a in cell cycle, differentiation, and apoptosis: a review. J Biochem Mol Toxicol. 2012 Feb;26(2):79-86.

3. Chan JA, Krichevsky AM, Kosik KS. MicroRNA-21 is an antiapoptotic factor in human glioblastoma cells. Cancer Res. 2005 Jul 15;65(14):6029-33.

4. $\quad$ Si ML, Zhu S, Wu H, Lu Z, Wu F, Mo YY. miR-21-mediated tumor growth. Oncogene. 2007 Apr 26;26(19):2799-803.

5. Sun D, Layer R, Mueller AC, Cichewicz MA, Negishi M, Paschal BM, et al. Regulation of several androgen-induced genes through the repression of the miR-99a/let-7c/miR-125b-2 miRNA cluster in prostate cancer cells. Oncogene. 2013 Mar 18.

6. Lan L, Ui A, Nakajima S, Hatakeyama K, Hoshi M, Watanabe R, et al. The ACF1 Complex Is Required for DNA Double-Strand Break Repair in Human Cells. Molecular Cell. [doi: DOI: 10.1016/j.molcel.2010.12.003]. 2010;40(6):976-87.

7. Nakamura K, Kato A, Kobayashi J, Yanagihara H, Sakamoto S, Oliveira DV, et al. Regulation of homologous recombination by RNF20-dependent H2B ubiquitination. Mol Cell. 2011 Mar 4;41(5):515-28.

8. Sheu JJ, Choi JH, Guan B, Tsai FJ, Hua CH, Lai MT, et al. Rsf-1, a chromatin remodelling protein, interacts with cyclin E1 and promotes tumour development. J Pathol. 2013 Mar;229(4):559-68.

9. $\quad$ Saito Y, Suzuki H, Tsugawa H, Nakagawa I, Matsuzaki J, Kanai Y, et al. Chromatin remodeling at Alu repeats by epigenetic treatment activates silenced microRNA-512-5p with downregulation of Mcl-1 in human gastric cancer cells. Oncogene. 2009 Jul 30;28(30):2738-44. 10. Tellez CS, Juri DE, Do K, Bernauer AM, Thomas CL, Damiani LA, et al. EMT and stem cell-like properties associated with miR-205 and miR-200 epigenetic silencing are early manifestations during carcinogen-induced transformation of human lung epithelial cells. Cancer Res. 2011 Apr 15;71(8):3087-97.

11. Li Y, Gao L, Luo X, Wang L, Gao X, Wang W, et al. Epigenetic silencing of microRNA193a contributes to leukemogenesis in $\mathrm{t}(8 ; 21)$ acute myeloid leukemia by activating the PTEN/PI3K signal pathway. Blood. 2013 Jan 17;121(3):499-509.

12. Fazi F, Racanicchi S, Zardo G, Starnes LM, Mancini M, Travaglini L, et al. Epigenetic silencing of the myelopoiesis regulator microRNA-223 by the AML1/ETO oncoprotein. Cancer Cell. 2007 Nov;12(5):457-66.

13. Alvarez-Saavedra M, Antoun G, Yanagiya A, Oliva-Hernandez R, Cornejo-Palma D, Perez-Iratxeta $\mathrm{C}$, et al. miRNA-132 orchestrates chromatin remodeling and translational control of the circadian clock. Hum Mol Genet. 2011 Feb 15;20(4):731-51.

14. Sakurai K, Furukawa C, Haraguchi T, Inada K, Shiogama K, Tagawa T, et al. MicroRNAs miR-199a-5p and -3p target the Brm subunit of SWI/SNF to generate a doublenegative feedback loop in a variety of human cancers. Cancer Res. 2011 Mar 1;71(5):1680-9. 15. Sun D, Lee YS, Malhotra A, Kim HK, Matecic M, Evans C, et al. miR-99 family of MicroRNAs suppresses the expression of prostate-specific antigen and prostate cancer cell proliferation. Cancer Res. 2011 Feb 15;71(4):1313-24.

16. Schramedei K, Morbt N, Pfeifer G, Lauter J, Rosolowski M, Tomm JM, et al. MicroRNA-21 targets tumor suppressor genes ANP32A and SMARCA4. Oncogene. 2011 Jun 30;30(26):2975-85.

17. Thoms J, Bristow RG. DNA Repair Targeting and Radiotherapy: A Focus on the Therapeutic Ratio. Seminars in Radiation Oncology. 2010;20(4):217-22. 
18. Orom UA, Derrien T, Beringer M, Gumireddy K, Gardini A, Bussotti G, et al. Long noncoding RNAs with enhancer-like function in human cells. Cell. 2010 Oct 1;143(1):46-58.

19. Orom UA, Derrien T, Guigo R, Shiekhattar R. Long noncoding RNAs as enhancers of gene expression. Cold Spring Harb Symp Quant Biol. 2010;75:325-31.

20. Lai F, Orom UA, Cesaroni M, Beringer M, Taatjes DJ, Blobel GA, et al. Activating

RNAs associate with Mediator to enhance chromatin architecture and transcription. Nature. 2013

Feb 17. 


\section{Appendix}

\section{Chapter 5}

\section{Unpublished results}

\section{Adapted from final report to the DOD for grant BC073568}

\section{O-GIcNAc Misregulation and Aneuploidy in Breast Cancer}

\section{Contribution:}

Adam Mueller designed and performed all experiments. Anindya Dutta supervised all aspects of the study.

\section{Introduction:}

\section{O-GIcNAc and Emi1 in genomic instability of breast cancer.}

One of the key steps in initiation and progression of breast cancer is the loss of control over DNA replication and the cell cycle that lead to genetic instability and aneuploidy. Gene amplification and transcriptional misregulation resulting from this instability represent some of the last steps in the formation of malignant neoplasias. O-linked $\mathrm{N}$-acetyl glucosamine is a posttranslational protein modification similar in many respects to protein phosphorylation in the number of targets that are modified and the variety of cellular pathways it modulates(1). An offshoot of the glycolysis pathway, it is thought to act as a nutrient sensor, its addition modulated by the availability of glucose. Other groups have shown that mis-regulation of O-GlcNAc can lead to faulty cell cycle regulation and aneuploidy in HeLa cells(2). Further defects in control of 
the cell cycle and DNA repair may act synergistically with O-GlcNAc regulatory defects to cause malignant transformation. We have shown that depletion of Early Mitotic Inhibitor 1(Emi1) leads to cell cycle defects and re-replication in normal breast epithelial cells(3). We have proposed to examine whether siRNA knockdown of Emil along with O-GlcNAc Transferase(OGT) and O-GlcNAcase(NCOAT) can act synergistically to cause large scale rereplication, aneuploidy and eventually lead to malignant transformation in breast epithelial cells.

\section{Determining the role of microRNAs in the response to DNA damage in breast cancer.}

A great deal of recent attention has been placed on the role of microRNAs in cancer, especially in regards to cell survival and proliferation. MicroRNAs are a recently discovered class of small RNA molecules that post-transcriptionally regulate the expression of a plethora of genes and cellular pathways. They play a role in the differentiation and function of numerous cell types across species, and some have been shown to dramatically affect cell survival( 4,5$)$. They have been shown to regulate both p53(6) and the estrogen receptor(7), and have the potential to contribute to the progression of breast cancer. They also represent excellent therapeutic targets, as they can now be readily introduced as well as inhibited both in cells in culture as well as in vivo in animals or patients(8). The function and regulation of miRNAs are collectively a focus of this lab, as is the role of genomic instability in cancer, and the examination of their potential role in the response of breast cancer to DNA damage ties into the goals of this project. We intended to identify microRNAs whose expression is modulated in response to DNA damage, as well as those differentially expressed between cancers with varying resistance to DNA damaging radiotherapy and chemotherapy, in order to to identify microRNAs that may be able to enhance normal breast epithelial cells ability to cope with re-replication stress as well as modulate cancer cells ability to withstand treatments. Expression of microRNAs targeting key components of the 
DNA damage response, cell cycle and DNA replication could have a significant effect on the level of response a breast cancer has to a treatment regimen, and introducing exogenous or blocking expressed miRNAs could confer potentially chemo or radiosensitivity to treatment refractory cases of cancer.

\title{
Results:
}

\section{Testing checkpoint activation, cell cycle effect and re-replication after co- depleting Emi1 and OGT or NCOAT}

\author{
siRNA depletion of OGT blocks Emi1 induced DNA re-replication in some breast cancer \\ cell lines.
}

Our first goal is to determine if Emil knockdown in conjunction with mis-regulation of O-GlcNAc post-translational modification of proteins can act synergistically to cause rereplication. We have obtained antibodies as well as siRNA oligos against OGT, NCOAT and Emi1. We treated MCF7 and Sk-Br-3 breast cancer cells as well as MCF10a breast mammary epithelial cell lines with 20nM siRNA oligos targeting various combinations of OGT, NCOAT, Emi1 and GL2 firefly luciferase using Invitrogen Lipofectamine RNAimax transfection reagent. The cells were collected 72 hours post-transfection and analyzed by propidium iodide flow assisted cell sorting(FACS) to determine the cell cycle profile and whether re-replication was occurring in these cells. We generated whole cell lysates from these samples as well to perform western analysis in order to confirm knockdown as well as examine cell cycle and checkpoint markers. 
In MCF10a and MCF7 we observed a substantial population of cells with greater than $4 \mathrm{~N}$ DNA content, characteristic of re-replication, with Emil treatment. This re-replication was largely abrogated by treatment with siRNA against OGT(fig 1a, 1b). We were only able to achieve a small degree of re-replication with SKBR3, making it difficult to determine the effect of OGT RNAi, but this effect was slightly enhanced by NCOAT knockdown(not shown). As shown in figure 1c, we achieved efficient knockdown of OGT, and the effect of OGT knockdown on re-replication was not through rescue of geminin levels, which are reduced due to increased APC activity. Interestingly, Cyclin A levels were reduced with OGT RNAi, this is noteworthy because co-depletion of geminin and Cyclin A are sufficient to cause re-replication in HeLa cells which are resistant to geminin induced re-replication.

\section{siRNA depletion of OGT blocks MLN4924 induced DNA re-replication.}

MLN4924 is a small molecule inhibitor that prevents an activating neddylation modification of cullins, that has been found to cause apoptosis in cancer cells through s-phase arrest or re-replication(9). Within 24 hours of treatment with MLN4924, we have been able to see significant re-replication in a number of cell lines, making it an efficient tool in studying rereplication mechanisms. We treated cells with siRNA against OGT for 48 hours prior to treating with MLN4924, and prepared the samples for PI FACS. As shown, OGT RNAi was sufficient to cause a substantial reduction in the number of re-replicating cells with MLN4924 treatment(Fig. 2a).

\section{OGT inhibition with ST060266 can block re-replication by MLN4924}

We obtained a small molecule inhibitor of OGT, ST060266 to determine if chemical inhibition of OGT could prevent re-replication initiated by MLN4924. We pretreated cells for 12 
hours with ST060266 prior to the addition of MLN4924. As shown, there were substantially

fewer re-replicating cells with OGT inhibition than with DMSO(fig2b).

\section{OGT inhibition has a modest effect on re-replication induced following release from thymidine block.}

In order to determine if OGT could inhibit re-replication through a mechanism other than blocking entry into S-phase, we treated cells for 12 hours with $2 \mathrm{mM}$ thymidine for 12 hours, GM for 12 hours, thymidine for 12 hours prior to releasing. We used HCT116 colon cancer cells due to their relative ease of synchronizing. 12 hours prior to release, we treated with Alloxan, an inhibitor of OGT. Cells were treated with MLN4924 8 hours prior to release, and released into MLN4924 and nocodazole. Cells were collected for FACS over 24 hours. As shown, at 24 hours, there was a modest decrease in the amount of MLN4924 induced re-replication that occurred when Alloxan was added(fig2c). This indicates that DNA re-replication may require the action of OGT following entry into S-phase.

\section{OGT knockdown halts DNA replication.}

We performed a BrdU ELISA to determine the rate of DNA synthesis following Emi1 and OGT RNAi. 72 hours following treatment with siRNA in 96 well plates, we examined BrdU incorporation. As shown, by 72 hours, Emil knockdown has resulted in an abrogation of BrdU incorporation likely secondary to checkpoint activation. A similarly dramatic reduction is seen with OGT inhibition(fig3a), even though the cells have a normal FACS profile. This would seem to indicate that O-GlcNAc modification is required for normal DNA synthesis as well as rereplication, and may regulate multiple phases of the cell cycle.

PCNA is candidate for modification with O-GlcNAc 
To identify substrates of OGT, we performed pull-down experiments with wheat germ agglutinin, a lectin that binds to O-GlcNAcated proteins. Cells were treated with Alloxan or DMSO for 24 hours prior to lysis. Lysates were incubated with WGA before washing and boiling with SDS loading buffer. Western blotting of the pull-downs were then performed for Cdt1, MCM2, CDK2 and PCNA, all proteins which are crucial for DNA replication. Of these, PCNA was found to associate with WGA, making it a likely candidate for regulation by OGT.

\section{Identifying microRNAs that regulate the DNA damage response that are differentially expressed between breast cancers}

In order to examine microRNAs that were involved in regulating the DNA damage response, we performed microarrays to detect miRNA expression in MCF7 and SKBR3 breast cancer cell lines following 24 hours of 5gy IR treatment. Mir99a was one of the microRNAs that was identified to be downregulated 2 fold in MCF7. Additionally, its basal expression level was found to be 2.2 fold higher in MCF7 than SKBR3. We examined the expression level of miR99a by qRT-PCR in MCF10a, SKBR3 breast cell lines, as well as p53+/+ and -/- HCT116 cells. We found expression to be significantly lower among the p53 mutant cell lines, however, we could not find any difference in expression following DNA damage(fig4a). Our lab has the additional interest in determining microRNAs that are differentially regulated over the course of prostate cancer progression, and miR99a was identified as a microRNA that was downregulated 5 fold in the androgen independent cell line C4-2, vs. the androgen dependent progenitor cell line LnCAP. We decided to examine the potential role of miR99a in the DNA damage response in both breast and prostate cancer cell lines. 


\section{Cdc25a is a target of miR-99a}

Cdc25a was found to be a predicted target of miR99a by multiple algorithms. The main function reported for Cdc25a is that its degradation following DNA damage inhibits S-phase progression as part of the cell's adaptive response(10). Targeting of Cdc25a by microRNAs could potentially regulate the kinetics and magnitude of the intra-s-phase checkpoint. To determine whether it is a target, we introduced exogenous miR99a into C4-2 cells and performed sucrose gradient fractionation to purify polyribosome and monoribosome associated mRNAs. When miR99a was introduced, we found Cdc25 shifted from the polyribosome fraction to the monosome fraction indicating that translation was inhibited(fig 4b, 6a). Additionally, when we introduce exogenous miR-99a into cells, we see a reduction in Cdc25a protein level to below that of irradiated cells(Fig 6c). Finally, we cloned the Cdc25a 3'UTR into a luciferase reporter vector and found that exogenous miR-99a could reduce luciferase expression by 50\%(Fig. 6d), an effect that was negated by mutation of the predicted miR-99a target site(shown Fig 6a). Taken together, these data show that Cdc25a is a target of miR-99a.

\section{miR99a decreases $3 \mathrm{H}$ thymidine incorporation following DNA damage}

To determine whether miR99a can alter the DNA damage response, we examined whether its introduction could alter tritium labeled thymidine incorporation following $10 \mathrm{gy}$ gamma irradiation in MCF10a cells. Prior to radiation treatment, cells were labeled with 14Cthymidine for 24 hours to normalize to cell number and basal growth rate. A 15 minute $3 \mathrm{H}-$ thymidine pulse was performed prior to each time point following radiation. We found a modest reduction in $3 \mathrm{H}$-thymidine incorporation at the 30 minute time point(fig4c). Additionally, we 
performed a dose titration of ionizing radiation in LnCAP and C4-2 cells in which miR99a had been introduced. With exogenous miR99a, we found a reduction in thymidine incorporation in both cell lines $2 \mathrm{hr}$ following IR, particularly in C4-2 which has low endogenous miR99a expression(fig5a,b), and robustly decreased 60' following 5gy IR in C4-2 cells(Fig. 6b).

\section{miR99a sensitizes C4-2 cells to DNA damage}

To determine whether introduction of miR99a can confer modulate a cells sensitivity to DNA damage, we introduced it into LnCAP and C4-2 cells which express it at different endogenous levels, and performed clonogenic survival assays following a dose titration of gamma irradiation. We found that in LnCAP cells, there was little effect on cell survival with the introduction of more miR99a(Fig. 5c), which was already highly expressed, however in C4-2, exogenous miR99a caused a marked decrease in cell survival(Fig. 5d), decreasing the colony numbers at the 2.5 and 5 gy doses to below the those seen in LnCAP, which was more sensitive than the control C4-2 cells. Interestingly, we see a similar sensitization with siRNA knockdown of Cdc25a(Fig. 6f), indicating this may be an important target of miR-99a in mediating radiation sensitivity of cancer cells. This data indicates that miR99a has the ability to modulate cells immediate response to DNA damage, as well as their long term survival following exposure to radiation, which may prove to be useful as a diagnostic indicator as well as perhaps a therapeutic target. 


\section{Conclusions:}

\section{O-GIcNAc and Emi1 in genomic instability of breast cancer.}

We were able to demonstrate that siRNA depletion of OGT was able to block DNA rereplication induced by knockdown of Emil. Additionally, we determined that re-replication induced by the cullin inhibitor MLN4924 was also reduced by siRNA of OGT. Chemical inhibition of OGT by ST060266 appeared to reduce this re-replication as well, but we were unable to determine that OGT activity was in fact reduced in vivo by this somewhat uncharacterized inhibitor. Short pretreatment with alloxan could not block rereplication induced by MLN4924, indicating that longer inhibition of OGT activity is necessary in order to block rereplication. We have also seen a dramatic reduction in BrdU incorporation with OGT inhibition, indicating its function is required for general DNA synthesis as well as re-replication to occur. The growth inhibition observed with OGT knockdown seems to point towards a more generalized effect on the cell cycle, where cells may be arrested throughout the cell cycle, which would appear to be normal by FACS analysis, but be unable to continue to divide. We also identified PCNA as a substrate for OGT, which being a critical component of the replication machinery would be a likely target for OGT regulation of DNA synthesis. The sheer number of substrates modified by OGT may make it difficult to determine exactly which one or many are responsible for this effect, but it may be quite useful to potentially target the enzyme itself as highly metabolically active and rapidly replicating tumors may be susceptible to this observed reduction in growth following OGT inhibition. 


\section{Determining the role of microRNAs in the response to DNA damage in breast cancer.}

We have identified a microRNA that is downregulated in more advanced breast and prostate cancer cell lines that appears to play a role in the DNA damage response. We have found that when exogenous miR99a is introduced into cells, Cdc25a shifts from the polysome to monosome fraction indicating its translation is being repressed. Expression of miR-99a reduces luciferase activity of a Cdc25a 3' UTR reporter, which is rescued by mutation of the miR-99a target site, validating Cdc25a is a direct target of miR-99a. Additionally introduction of miR99a causes a further decrease in $3 \mathrm{H}$-thymidine incorporation following irradiation of breast and prostate cancer cells. Introduction of miR99a into a cell line where its expression is typically low causes a decrease in its ability to form colonies following treatment with ionizing radiation. 


\section{References:}

1. Zachara NE, Hart GW. O-GlcNAc a sensor of cellular state: the role of nucleocytoplasmic glycosylation in modulating cellular function in response to nutrition and stress. Biochim Biophys Acta. 2004 Jul 6;1673(1-2):13-28.

2. Slawson C, Zachara NE, Vosseller K, Cheung WD, Lane MD, Hart GW. Perturbations in O-linked beta-N-acetylglucosamine protein modification cause severe defects in mitotic progression and cytokinesis. J Biol Chem. 2005 Sep 23;280(38):32944-56.

3. Machida YJ, Dutta A. The APC/C inhibitor, Emi1, is essential for prevention of rereplication. Genes Dev. 2007 Jan 15;21(2):184-94.

4. Lee DY, Deng Z, Wang CH, Yang BB. MicroRNA-378 promotes cell survival, tumor growth, and angiogenesis by targeting SuFu and Fus-1 expression. Proc Natl Acad Sci U S A. 2007 Dec 18;104(51):20350-5.

5. Medina R, Zaidi SK, Liu CG, Stein JL, van Wijnen AJ, Croce CM, et al. MicroRNAs 221 and 222 bypass quiescence and compromise cell survival. Cancer Res. $2008 \mathrm{Apr}$ 15;68(8):2773-80.

6. Park SY, Lee JH, Ha M, Nam JW, Kim VN. miR-29 miRNAs activate p53 by targeting p85 alpha and CDC42. Nat Struct Mol Biol. 2009 Jan;16(1):23-9.

7. Zhao JJ, Lin J, Yang H, Kong W, He L, Ma X, et al. MicroRNA-221/222 negatively regulates estrogen receptor alpha and is associated with tamoxifen resistance in breast cancer. $\mathrm{J}$ Biol Chem. 2008 Nov 7;283(45):31079-86.

8. Gondi CS, Rao JS. Concepts in in vivo siRNA delivery for cancer therapy. J Cell Physiol. 2009 Aug;220(2):285-91.

9. Swords RT, Kelly KR, Smith PG, Garnsey JJ, Mahalingam D, Medina E, et al. Inhibition of NEDD8-activating enzyme: a novel approach for the treatment of acute myeloid leukemia. Blood. 2010 May 6;115(18):3796-800.

10. Bartek J, Lukas J. Mammalian G1- and S-phase checkpoints in response to DNA damage. Curr Opin Cell Biol. 2001 Dec;13(6):738-47. 


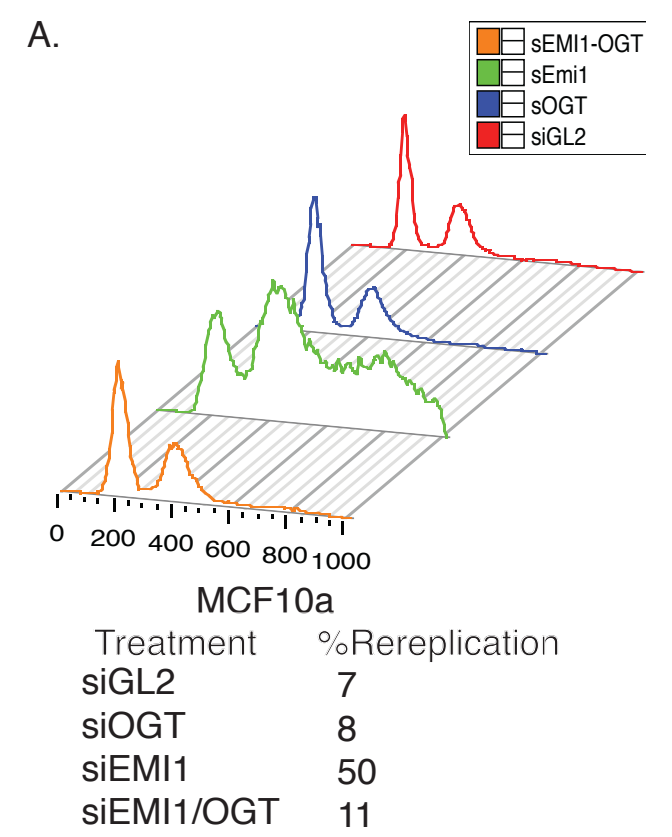

C.

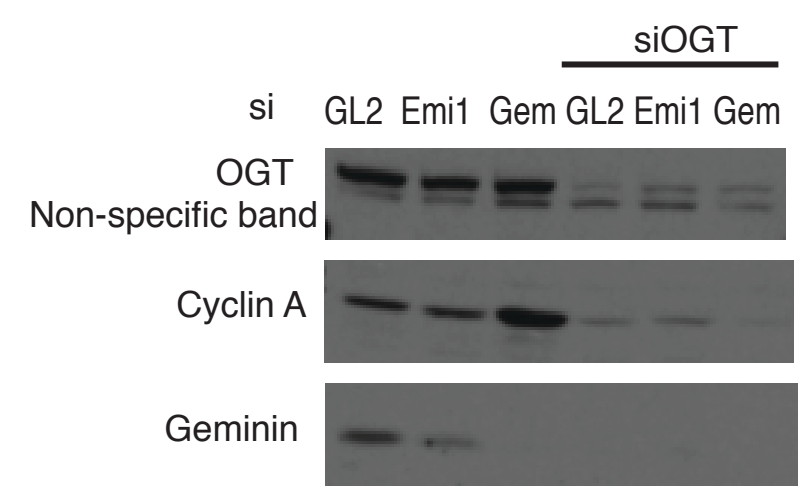

B.
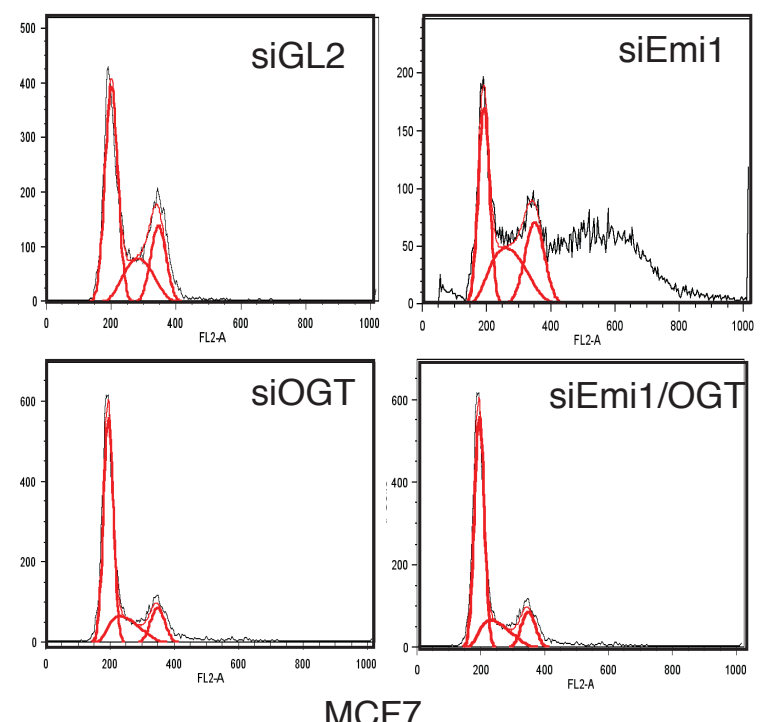

Treatment \%Rereplication

siGL2

SIOGT $\quad 10$

SiEMI1 50

SIEMI1/OGT 10

Figure 1. OGT depletion abrogates the rereplication induced by Emi1 depletion. A-B.

Propidium Iodide FACS of MCF10a(A) and MCF7(B) cells 72 hours following treatment with siRNA targeting Emi1, OGT or both. \%rereplication refers to the percent of total cells with $>4 \mathrm{~N}$ DNA content. 10000 cells were counted per sample. C. Western blot for OGT, Cyclin A and Geminin. Cells were treated for 72 hours with siRNA targeting Emi1, Geminin, OGT or OGT and either Emil or Geminin. 
A.
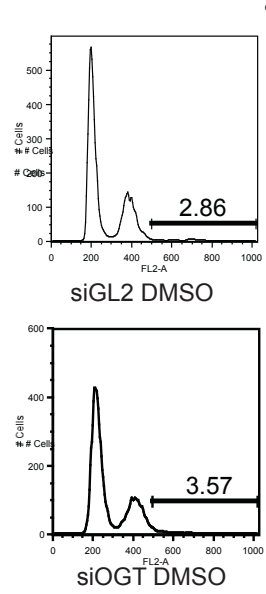

101314
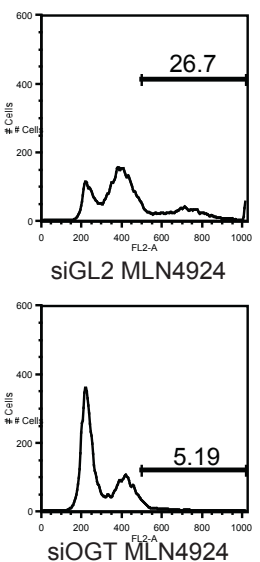

B.
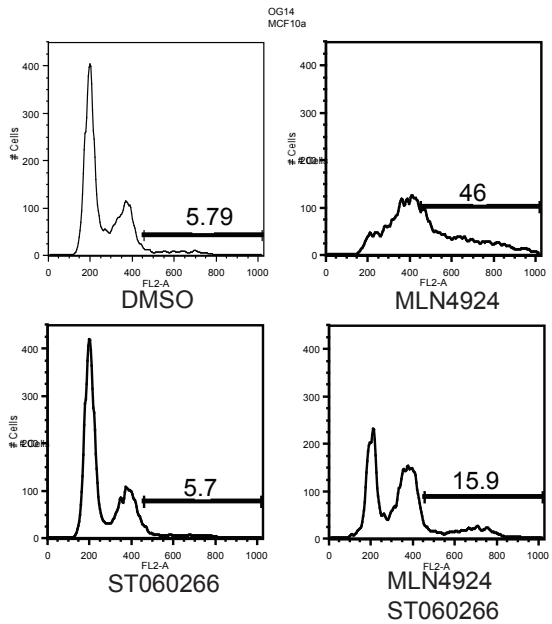

C.
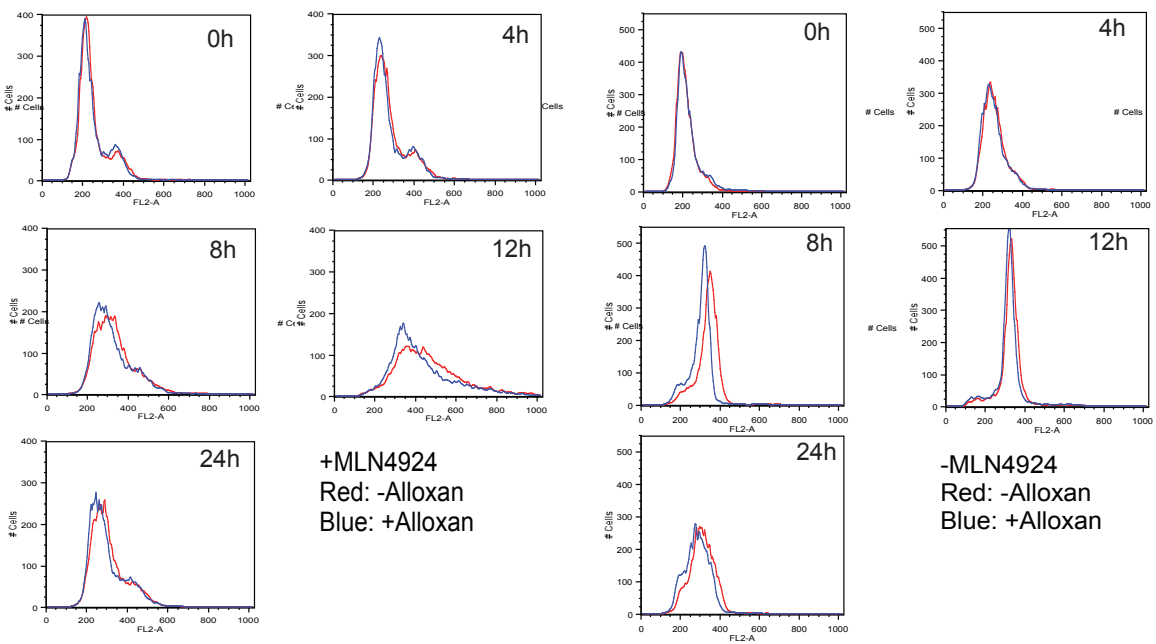

-MLN4924

Red: -Alloxan Blue: +Alloxan

Figure 2. siRNA or small molecule inhibition of OGT can prevent rereplication. A. Cell cycle profile and \%re-replication of MCF10a cells following siOGT and MLN4924 treatment. Cells were transfected with indicated siRNA oligo and treated with the indicated drug for 72 hours and then analyzed by PI FACS for cell cycle profile. Number indicates percentage of total cells with $>4 \mathrm{~N}$ DNA content.B. Cell cycle profile and \%re-replication following treatment with MLN4924 and OGT inhibitor ST060266. Cells treated with the indicated drug for 72 hours and then analyzed by PI FACS for cell cycle profile. Number indicates percentage of total cells with $>4 \mathrm{~N}$ DNA content. C. Cell cycle profile of HCT116 cells following double thymidine block and release with MLN4924 and OGT inhibition by Alloxan(C), red=DMSO, blue=Alloxan, + MLN left panel, -MLN right panel. Cells were blocked twice in thymidine for 12 hours, with a 12 hour release in between treatments to synchronize the cell population to the G1/S transition. Cells were also treated with Alloxan or DMSO for 24 hours prior to release. After second thymidine treatment, cells were released, and cell cycle profile was analyzed by PI FACS. 
A.

BrdU incorporation following $72 \mathrm{hrs} \mathrm{RNAi}$
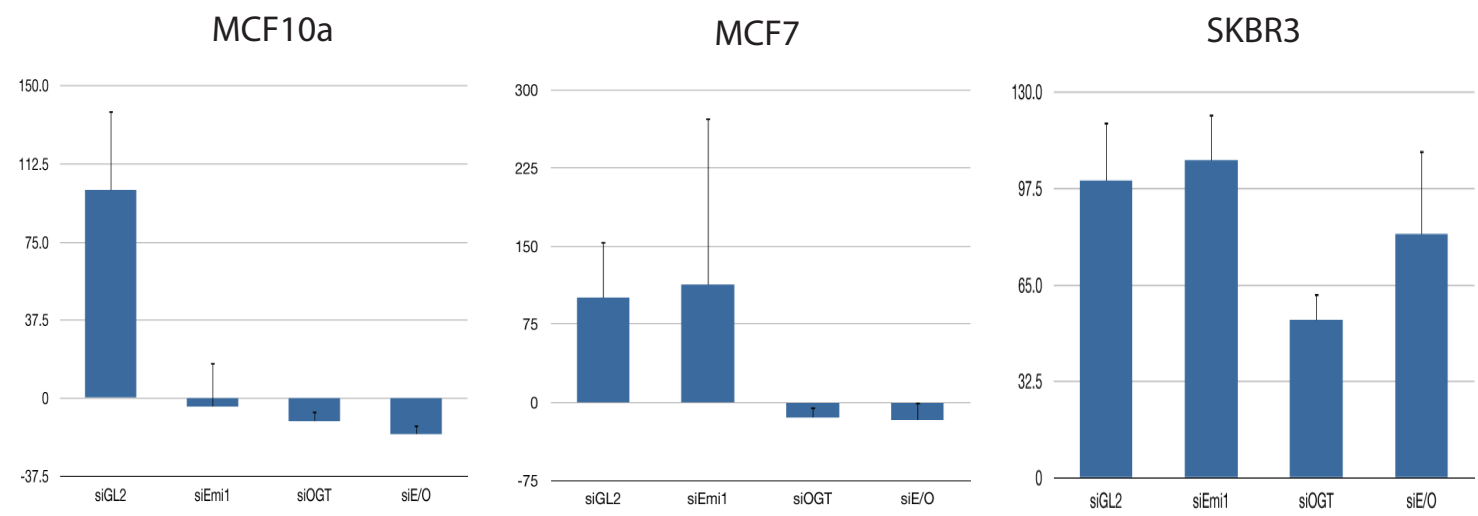

B.

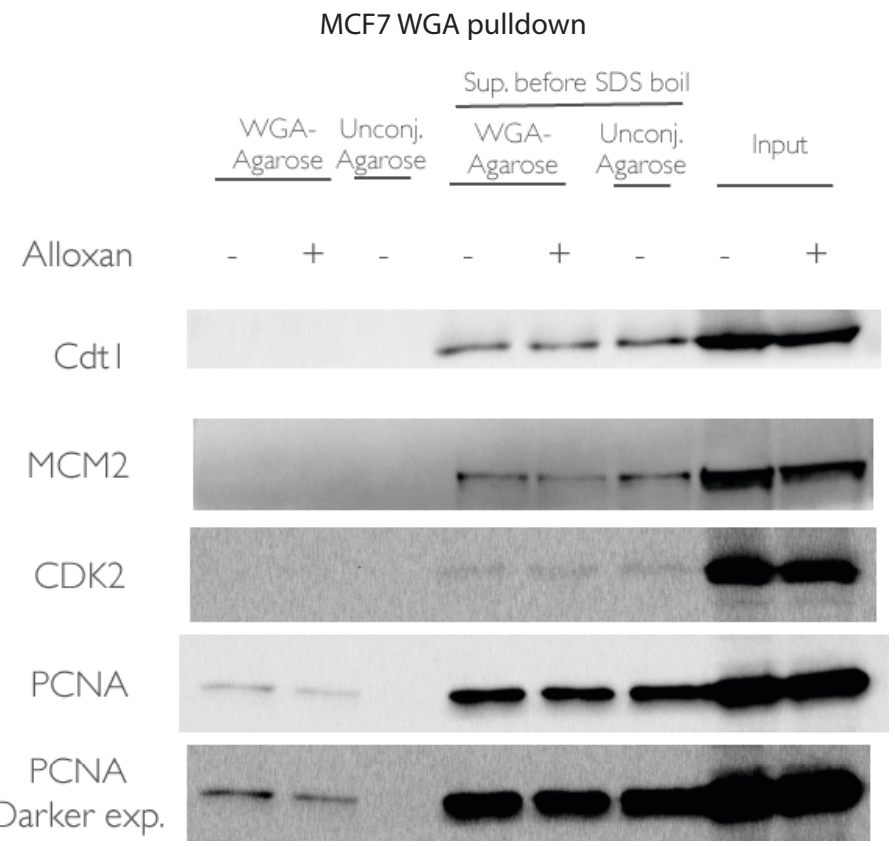

Figure 3. OGT reduces BrdU incorporation, and modifies cell cycle proteins. A. BrdU incorporation in MCF10a, MCF7 and SKBR3 following siRNA targeting Emi1, OGT and both. Cells were incubated for 72 hours with indicated siRNAs and BrdU incorporation was measured by ELISA. Data represents mean +/- SD. N=3. B. Western blots for DNA replication and Sphase entry cell cycle proteins with O-GlcNAc modifications. O-GlcNAcylated proteins were pulled down with WGA agarose beads and visualized by western blotting. 
A.

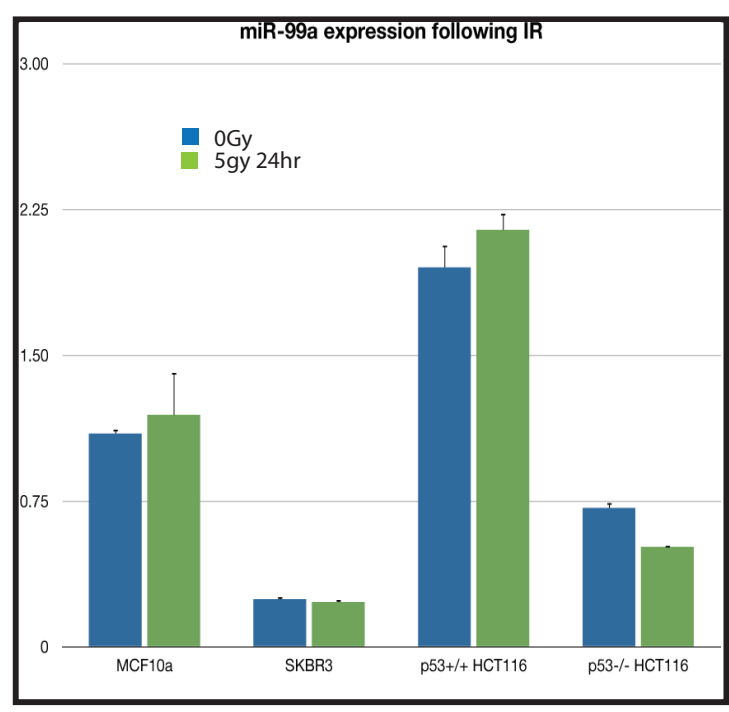

B.

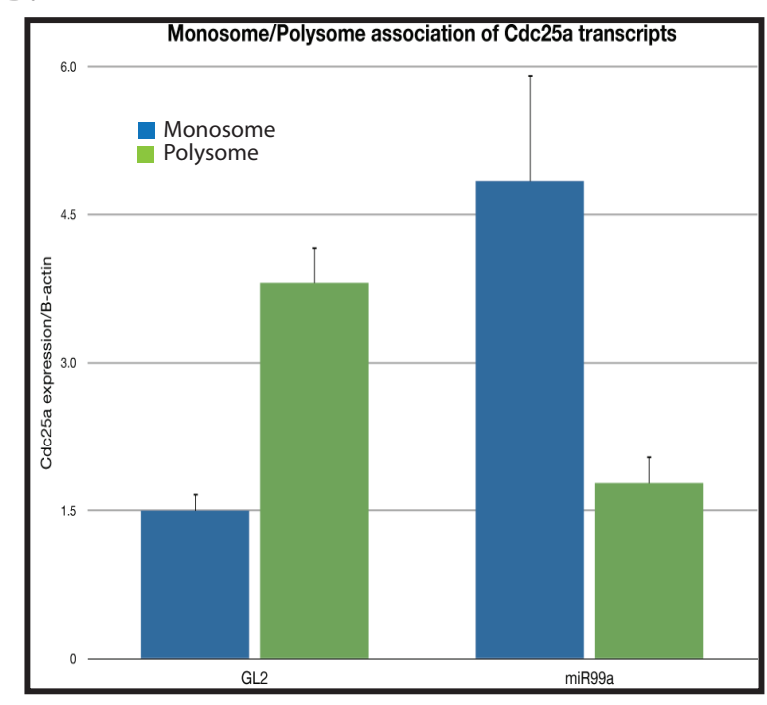

C. MCFIOa IOgy IR time course $3 \mathrm{H} / / 4 \mathrm{C}$ inc.

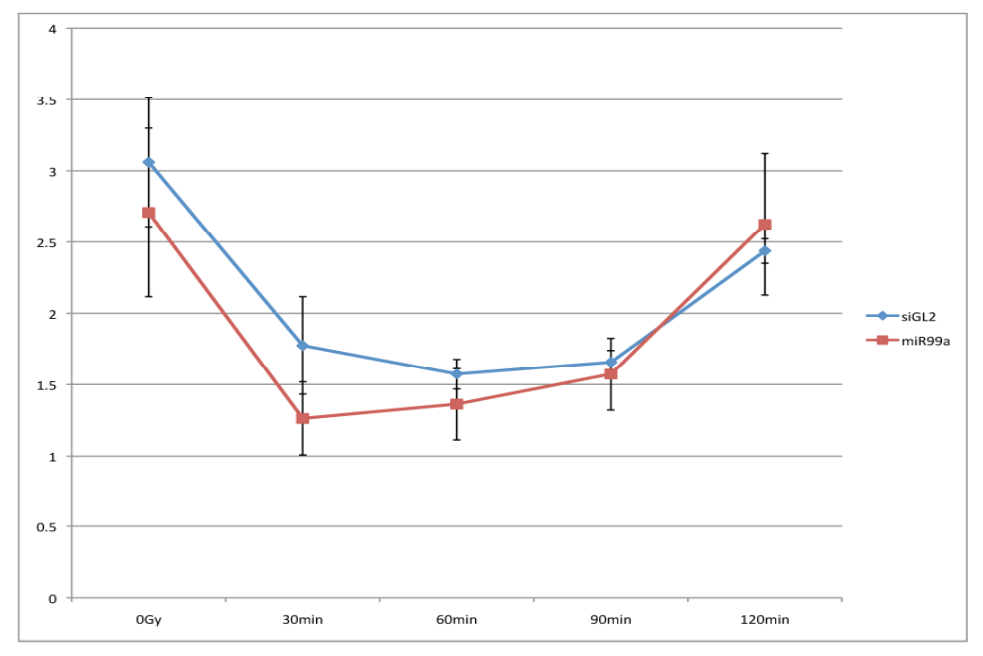

Figure 4. miR-99a not upregulated in all cell lines, but targets $C$ dc25a when introduced exogenously. A. qPCR analysis of miR99a expression in breast and colon cancer cell lines. Indicated cell lines were treated with 5 gy of ionizing radiation for 24 hours and miR99a expression was measured by qPCR. B. Polysome fractionation of C4-2 cells and qPCR of Cdc25a associated with ribosomal fractions following miR99a introduction. C4-2 cells were transfected with siGL2 or miR-99a for 48 hours, lysed and sucrose gradient fractionation of monoribosome and polyribosome fractions was performed. Data represents mean $+/-\mathrm{SD}, \mathrm{N}=3$. C . $3 \mathrm{H}-$ Thymidine incorporation time course following IR in MCF10a in the presence of miR-99a transfection. miR-99a was transfected in MCF10a cells and 3H-thymidine incorporation was measured at the indicated time points. Data represents mean $+/-\mathrm{SD}, \mathrm{n}=3$. 
A.

LnCAP $3 \mathrm{H}$-thymidine incorporation $2 \mathrm{hr}$ post IOgy IR

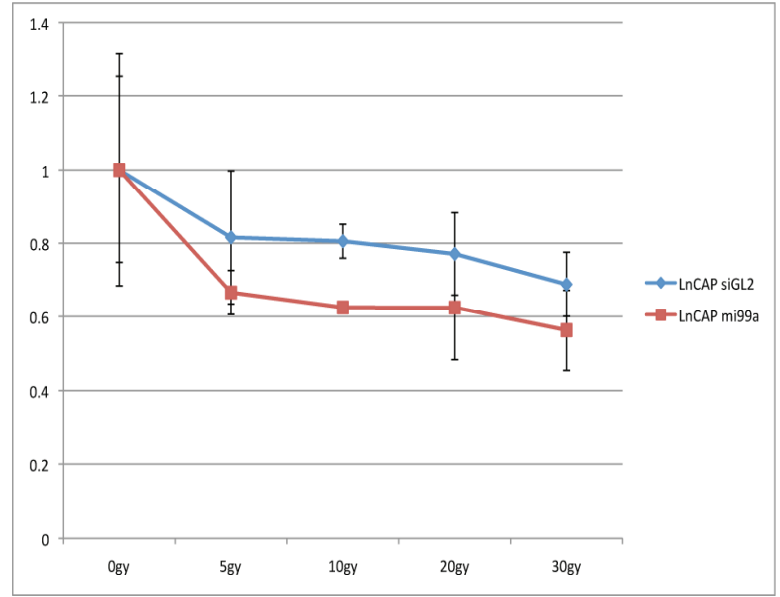

C.

LnCAP colony formation 9 days following $\mathbb{R}$

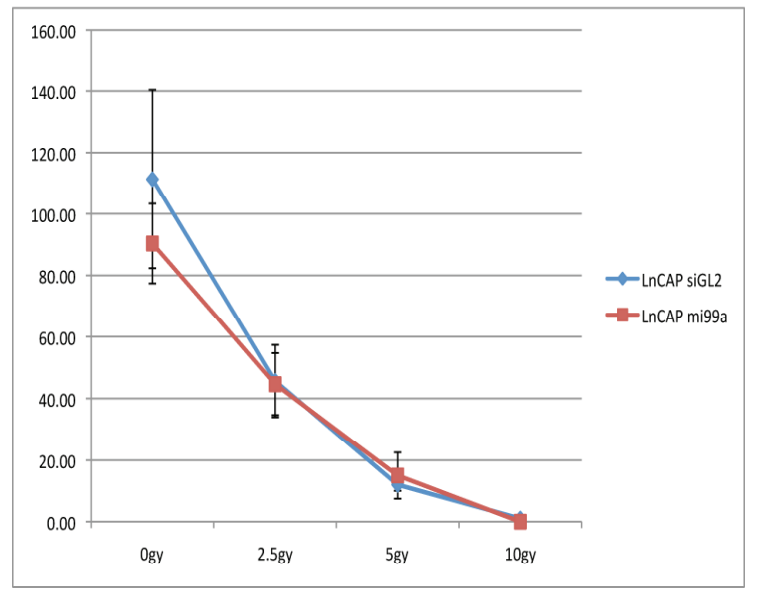

B.

C4-2 3H-thymidine incorporation 2hr post IOgy IR

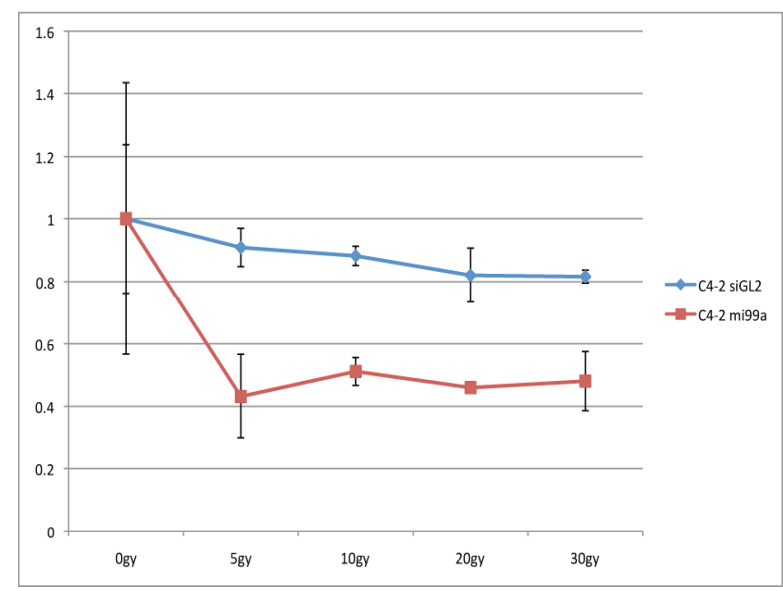

D. C4-2 colony formation 9 days following $\mathbb{R}$

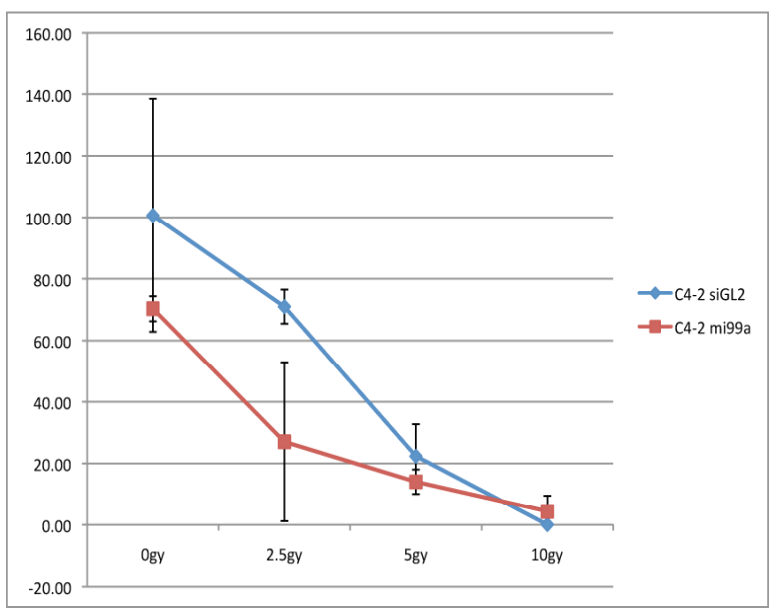

Figure 5. miR-99a can decrease cell proliferation in prostate cancer cells after IR treatment, and sensitizes C4-2 cells to DNA damage.A-B. Thymidine incorporation following IR dose titration in LnCAP and C4-2 cells. Cells were treated with the indicated dose of IR and thymidine incorporation was measured 2 hours following treatment. Data represents mean +/-SD, n=3.C-D. LnCAP and C4-2 clonogenic survival +/- miR99a following IR. Cells were treated with the indicated doses of IR, and number of colonies formed were measured 9 days following treatment. Data represents mean $+/-\mathrm{SD}, \mathrm{n}=3$. 
Figure 6

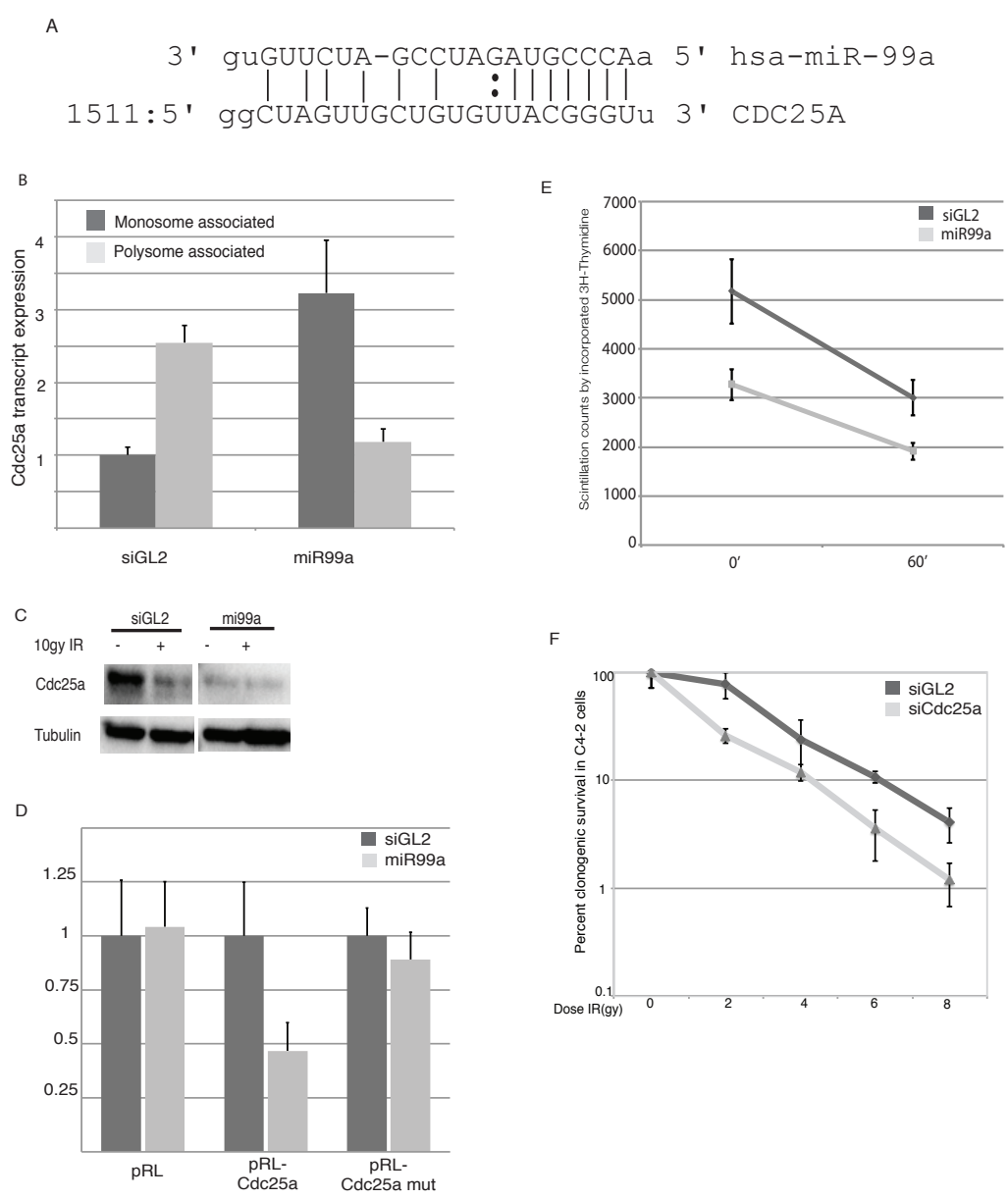

Figure 6. Cdc25a is a direct target of miR99a. A. miR-99a target sequence of Cdc25a 3'UTR. B. Polysome fractionation of C4-2 cells and qPCR of Cdc25a associated with ribosomal fractions following miR99a introduction. C4-2 cells were transfected with siGL2 or miR-99a for 48 hours, lysed and sucrose gradient fractionation of monoribosome and polyribosome fractions was performed. Data represents mean $+/-\mathrm{SD}, \mathrm{N}=3$.C. Cdc25a protein expression following introduction of exogenous miR-99a +/- ionizing radiation. Cells were transfected with siGL2 or miR-99a for 48 hours and treated with 10gy IR prior to lysis and western blotting 60 minutes following treatment.D. Cdc25a 3'UTR coupled luciferase activity following miR-99a introduction. Cells were transfected with siGL2 or miR-99a for 24 hours, prior to transfection with the luciferase constructs containing the Cdc25a 3' UTR and the 3'UTR with the miR-99a target site mutated, as well as pGL3. 24 hours later luciferase activity was measured. Renilla luciferase activity is plotted in relation to firefly luciferase activity. Data represents mean $+/-\mathrm{SD}$, $\mathrm{N}=3$.E. Thymidine incorporation following miR-99a introduction. Cells were transfected with siGL2 or miR-99a and treated with 5gy IR. Thymidine incorporation was then measured at the indicated time points. Data represents mean $+/-\mathrm{SD}, \mathrm{N}=3$.F. Clonogenic survival following IR with Cdc25a siRNA. C4-2 cells were transfected with either siGL2 or siCdc25a and treated with the indicated doses of IR. 9 days following IR treatment, the number of colonies per sample were measured. Data represents mean $+/-\mathrm{SD}, \mathrm{N}=3$. 